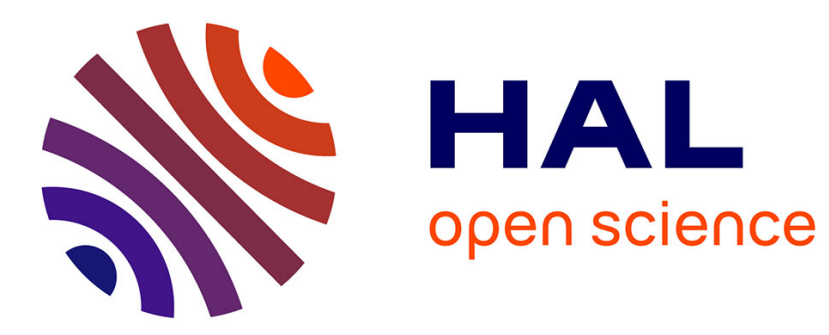

\title{
High order asymptotics for wave propagation across thin periodic interfaces
}

Bérangère Delourme, Xavier Claeys

\section{To cite this version:}

Bérangère Delourme, Xavier Claeys. High order asymptotics for wave propagation across thin periodic interfaces. 2012. hal-00682386

\section{HAL Id: hal-00682386 \\ https://hal.inria.fr/hal-00682386}

Preprint submitted on 25 Mar 2012

HAL is a multi-disciplinary open access archive for the deposit and dissemination of scientific research documents, whether they are published or not. The documents may come from teaching and research institutions in France or abroad, or from public or private research centers.
L'archive ouverte pluridisciplinaire HAL, est destinée au dépôt et à la diffusion de documents scientifiques de niveau recherche, publiés ou non, émanant des établissements d'enseignement et de recherche français ou étrangers, des laboratoires publics ou privés. 


\title{
High order asymptotics for wave propagation across thin periodic interfaces
}

\author{
Xavier Claeys $^{a}$, Bérangère Delourme $e^{b, c, d}$ \\ $a$ : Université de Toulouse, ISAE, Toulouse, France \\ $b$ : INRIA Rocquencourt, Domaine de Voluceau, BP 105 78153, Le Chesnay Cedex, France \\ $c$ : INRIA Saclay Ile de France and Ecole Polytechnique, CMAP, Route de Saclay, 91128, Palaiseau Cedex, France \\ $d$ : CEA, LETI, MINATEC, F38054, Grenoble, France
}

Corresponding author: Bérangère Delourme, ACM Caltech, Pasadena, USA

Address: 1200 E California Blvd, Pasadena, California 91106.

E-mail: delourme@ caltech.edu

tel: 626-395-3531

\begin{abstract}
This work deals with the scattering of acoustic waves by a thin ring that contains many regularly-spaced heterogeneities. We provide and justify a complete description of the solution with respect to the period and the thickness of the heterogeneities. Our approach mixes matched asymptotic expansions and homogenization theory.
\end{abstract}

Keywords

periodic thin interfaces, matched asymptotic expansions, homogenization, Helmholtz equation.

\section{Introduction}

This work is dedicated to the study of asymptotic models associated with electromagnetic wave scattering from thin rings that contain regularly spaced heterogeneities. We use asymptotic analysis techniques to study a case where the thickness of the ring and the distance between two consecutive heterogeneities (of size $\delta$ ) are very small compared to the wavelength of the incident wave and the diameter of the ring, which yields a precise description of the behavior of the solution as the thickness of the ring goes to 0 .

Without being exhaustive, let us indicate some works from the mathematical literature that share similarities with our problem. Many papers deal with the construction of approximate boundary conditions for the scattering by a perfect conductor coated by a periodic layer or by a periodic rough boundary. The construction of such approximate conditions relies on an asymptotic expansion of the solution with respect to the thickness and the period of the layer. Concerning electromagnetic scattering by impenetrable objects with rough boundaries, the first two terms of the expansion have been derived by Y.Achdou [2] and M. Artola and M. Cessenat [5] for the Maxwell equations in planar geometries. This work has been extended to the case of a circular geometry by A.Zebic [34] and to the case of general smooth geometries by T. Abboud and H. Ammari [1] for the Helmholtz equation. Higher order terms have been derived by A.L Madureira and F.Valentin [22] for the Laplace problem and by A.Bendali and J.R. Poirier [26] for the Helmholtz equation. In addition, M. Dauge, E. Faou, V. Péron [12] and H. Haddar, P. Joly and H.M. Nguyen [16] have derived asymptotic expansions in the case of strongly absorbing (non-rough) obstacles. Similar methods have been applied to the case of an impenetrable object coated by a thin dielectric layer, see [4, 31].

In the present article we are particularly interested in problems involving wave transmission through a rough thin dielectric layer. Although this case has already been studied in [14, 9, 8, 7] for thin perforated plates and periodic rough thin dielectric layers, only low order asymptotics have been derived so far 
(expansions up to 2nd order). Note that Reference [13] deals with the same configuration as the present paper. However, the purpose is different since it mainly focuses on the construction of stable approximate transmission conditions up to order 2 - only the first two terms of the asymptotic expansion are derived. Note that high order asymptotics for wave transmission through thin dielectric layer have already been derived by K. Schmidt and S. Tordeux in [30], but this work did not consider rapidly oscillating material characteristics.

Our problem is similar to the one considered in [30] (i.e. a 2-D wave transmission through a thin dielectric layer) with, in addition, fast oscillations in the material characteristics of the layer. We derive an asymptotic expansion up to any order, we provide error estimates for it, and obtain explicit approximate transmission conditions at any order (which is different from [13] that only considers order 2 asymptotics). More precisely, denoting $u_{\delta}(r, \theta)$ the solution to our problem (with $\left|r-r_{*}\right|$ the distance to the layer, and $\theta$ the longitudinal coordinate) we propose an expansion of the form

$$
\begin{array}{ll}
u_{\delta}(r, \theta)=\sum_{n=0}^{+\infty} \delta^{n} u_{n}(r, \theta) & \text { away from the layer, } \\
u_{\delta}(r, \theta)=\sum_{n=0}^{+\infty} \delta^{n} U_{n}\left(\frac{r-r_{*}}{\delta} \frac{r_{*} \theta}{\delta}, \theta\right) & \text { in the neighbourhood of the layer. }
\end{array}
$$

and provide error estimates for these expansions: there are the main results of the present article, see Theorem 10.1 and Theorem 10.2 .

The expansion above is not the same in the vicinity of the layer, and away from it: this is a boundary layer phenomenon. Moreover, the expansion close to the layer involves multiple scales inherited from the fast oscillating structure of the equations. Actually, we mix homogeneization techniques with matched asymptotics - we believe that this is a remarkable feature of the present work.

Homogeneization theory was developed by A. Bensoussan, J.L. Lions, G. Papanicolaou and E. SanchezPalencia [6, 29, 28] to analyze materials with fast oscillating characteristics. Matched asymptotics mainly originated from the work of Van Dyke [33] to treat boundary layer problems arising in fluid mechanics. While the analysis proposed by Van Dyke was formal, rigorous theory for the method of matched expansions was developed later on by the Russian school. Main general references [23, 18, 17, 15, 19, 20] provide rigorous matched asymptotics for a wide variety of singularly perturbed elliptic problems (wave transmission through thin dielectric layer is not considered though) with particular attention to perturbations centered around points with all kinds of boundary conditions: domains containing small inclusions, or with rounded corners on the boundary are typical examples.

The remainder of this report is organized as follows. In Section 1, we describe the setting of the problem and introduce the "Ansatz" of the asymptotic expansion. In Section 2 and 3, we derive the equations satisfied by the far field and near field terms of the matched asymptotic expansion of the solution. Section 4 is devoted to the study of Laplace problems in a normalized strip which allows us to explicit the behavior of the near field terms at infinity in Section 5 and then to write the matching conditions in Section 6. In Section 7, we prove the existence and uniqueness of the far and near field terms of the asymptotic expansion. In particular, we obtain in Section 8 semi-explicit formulas which uncouple far and near field problems (Theorem 8.3 is also a remarkable result of the present paper). The last two sections are devoted to the justification of the asymptotic expansion by constructing first a global expansion and then by establishing error estimates.

\section{Setting of the problem}

As a model problem we consider the propagation in harmonic regime of an electromagnetic wave in a medium that is invariant in one direction of space. We focus on the TE mode of the field that is ruled by the following 2-D Helmholtz equation,

$$
\operatorname{div}\left(\epsilon_{\delta}^{-1} \nabla u_{\delta}\right)+\omega^{2} \mu_{\delta} u_{\delta}=-f \quad \text { in } \mathbb{R}^{2} \quad \text { and } \quad \lim _{r \rightarrow \infty} \sqrt{r}\left(\partial_{r} u_{\delta}+i \omega u_{\delta}\right)=0
$$

where $\omega$ refers to the pulsation of the time variation and $f \in \mathrm{L}^{2}\left(\mathbb{R}^{2}\right)$ refers to a source function for which there exists $r_{e}>0$ such that $f(\mathbf{x})=0$ for $|\mathbf{x}|>r_{e}$. Besides $(r, \theta)$ denote the polar coordinates in $\mathbb{R}^{2}$. The 
characteristics of the medium of propagation are defined in a very specific manner, as follows.

The medium of propagation The functions $\epsilon_{\delta}(\mathbf{x})$ and $\mu_{\delta}(\mathbf{x})$ are assumed to be non-negative and defined over $\mathbb{R}^{2}$, referring respectively to the permittivity and permeability. Both are assumed to be constant except inside a ring of thickness $\delta$ centered around the origin (see Figure 1): there exist $r_{*} \in\left(0, r_{e}\right)$ and $\epsilon_{\infty}, \mu_{\infty}, c_{\infty}>0$ independent of $\delta$ such that

$$
\epsilon_{\infty} \mu_{\infty}=\frac{1}{c_{\infty}^{2}} \quad \text { and } \quad \epsilon_{\delta}(r, \theta)=\epsilon_{\infty}, \quad \mu_{\delta}(r, \theta)=\mu_{\infty} \quad \text { for } \quad\left|r-r_{*}\right|>2 \pi \delta .
$$

In this problem, the geometric parameter $\delta>0$ is assumed to be much smaller than the wavelength far from the heterogeneities: $\delta \omega / c_{\infty} \rightarrow 0$. Inside the ring $\left\{\left|r-r_{*}\right| \leq \delta / 2\right\}$ the functions $\epsilon_{\delta}(\mathbf{x})$ and $\mu_{\delta}(\mathbf{x})$ are assumed to have a periodic structure: the ring contains many small heterogeneities regularly placed according to the azimuthal $\theta$-direction. We suppose that the number $N$ of heterogeneities is related to the thickness of the ring as follows

$$
\delta=\frac{r_{*}}{N} \quad \text { where } \quad N \in \mathbb{N} \quad \text { and } \quad N \rightarrow \infty .
$$

Such a relation implies that $\delta$ only takes discrete values, so that the expression " $\delta \rightarrow 0$ " should be understood in the sense of (2). Inside the thin ring, we assume that the permeability and permittivity are then given by

$$
\epsilon_{\delta}(r, \theta)=\epsilon\left(\frac{r-r_{*}}{\delta}, \theta \frac{r_{*}}{\delta}\right) \quad \text { and } \quad \mu_{\delta}(r, \theta)=\mu\left(\frac{r-r_{*}}{\delta}, \theta \frac{r_{*}}{\delta}\right),
$$

where $\epsilon, \mu \in \mathrm{L}_{\text {loc }}^{2}\left(\mathbb{R}^{2}\right)$ are independent of $\delta$. Since $(r, \theta)$ are polar coordinates, for such a definition to be meaningful, it is necessary that $\epsilon$ and $\mu$ satisfy some periodicity assumption, see Fig 1 (b). We have to assume in addition that

$$
\left\{\begin{array} { l l } 
{ \epsilon ( \nu , s + 2 \pi ) = \epsilon ( \nu , s ) , } \\
{ \mu ( \nu , s + 2 \pi ) = \mu ( \nu , s ) , }
\end{array} \quad \text { and } \quad \left\{\begin{array}{ll}
\epsilon(\nu, s)=\epsilon_{\infty} & \text { if }|\nu|>\pi, \\
\mu(\nu, s)=\mu_{\infty} & \text { if }|\nu|>\pi,
\end{array}\right.\right.
$$

$$
\text { where } \quad s=\theta \frac{r_{*}}{\delta} \quad \text { and } \quad \nu=\frac{r-r_{*}}{\delta} .
$$

We also make standard assumptions on the bounds for material properties

$$
\exists \alpha>0 \quad \text { such that } \quad \alpha<\epsilon(\nu, s)<\frac{1}{\alpha} \quad \text { and } \quad \alpha<\mu(\nu, s)<\frac{1}{\alpha} \quad \forall \nu, s \in \mathbb{R} .
$$

To sum up, with the preceding definitions, the medium of propagation is everywhere homogeneous except inside a thin ring. Besides, as $\delta \rightarrow 0$, this ring of heterogeneities gets close to the following limit circle,

$$
\Gamma:=\left\{\mathbf{x} \in \mathbb{R}^{2},|\mathbf{x}|=r_{*}\right\} .
$$

Reformulation as a problem posed in a bounded domain In order to analyze Problem (1), we will need to reformulate it as a problem posed in a bounded domain denoted $\Omega$ defined as follows

$$
\begin{aligned}
& \Omega=\Omega_{-} \cup \Gamma \cup \Omega_{+} \\
& \text {with } \Omega_{+}:=\left\{\mathbf{x} \in \Omega \text { s.t. } r_{*}<|\mathbf{x}|<r_{e}\right\} \quad \text { and } \Omega_{-}:=\left\{\mathbf{x} \in \Omega \text { s.t. }|\mathbf{x}|<r_{*}\right\}
\end{aligned}
$$

so that $\epsilon_{\delta}=\epsilon_{\infty}$ and $\mu_{\delta}=\mu_{\infty}$ outside $\Omega$. We restate the Sommerfeld radiation condition as a condition set on $\partial \Omega$ by means of a Dirichlet-to-Neumann map. Such an operator can be defined by the formula

$$
T u=-\sum_{p=-\infty}^{+\infty} u_{p} k \frac{H_{|p|}^{(1) \prime}\left(k r_{e}\right)}{H_{|p|}^{(1)}\left(k r_{e}\right)} e^{i p \theta} \quad \text { where } \quad u_{p}=\frac{1}{2 \pi} \int_{0}^{2 \pi} u\left(r_{e}, \theta\right) e^{-i p \theta} d \theta
$$


It is well known that $T$ is continuous as an operator from $\mathrm{H}^{\frac{1}{2}}(\partial \Omega)$ to $\mathrm{H}^{-\frac{1}{2}}(\partial \Omega)$. Moreover the Sommerfeld radiation condition $\lim _{r \rightarrow \infty} \sqrt{r}\left(\partial_{r} u_{\delta}+i \omega u_{\delta}\right)=0$ is then equivalent to $\partial_{r} u_{\delta}+T u_{\delta}=0$ on $\partial \Omega$. Such a formulation of the radiation condition allows to rewrite (1) as a variational problem set on the bounded domain $\Omega$, as follows

$$
\begin{aligned}
& \text { Find } u_{\delta} \in \mathrm{H}^{1}(\Omega) \quad \text { such that } \quad a_{\delta}\left(u_{\delta}, v\right)=\int_{\Omega} f \bar{v} d \mathbf{x} \quad \forall v \in \mathrm{H}^{1}(\Omega), \\
& \text { where } a_{\delta}(u, v)=\int_{\Omega}\left(\epsilon_{\delta}^{-1} \nabla u \cdot \nabla \bar{v}-\omega^{2} \mu_{\delta} u \bar{v}\right) d \mathbf{x}+\int_{\partial \Omega} \bar{v} T u d \sigma .
\end{aligned}
$$

It is well-known that this variational formulation is well posed, see for example [11]. The bilinear form $a_{\delta}(\cdot, \cdot)$ actually satisfies inf - sup conditions uniformly with respect to $\delta$ as will be shown in Proposition 10.3 .

\subsection{General methodology and main results}

Our purpose is to describe the terms of the expansion of $u_{\delta}$ for $\delta \rightarrow 0$. In our case, due to the fast variations of $\epsilon_{\delta}, \mu_{\delta}$ with respect to the angular coordinate, it does not seem possible to write a uniform expansion of the solution in the whole domain $\Omega$. Roughly speaking, the solution $u_{\delta}$ oscillates rapidly in a region confined to the vicinity of the periodic ring: this is a boundary layer phenomenon. We use matched asymptotics to cope with this. We first give a brief review on how to apply this method in the present context which, we hope, will help the reader understanding our approach. We follow five steps.

Step I: Far field ansatz (Section 2): we start from a guess of the general form (called "ansatz") of the expansion of $u_{\delta}$ both in $\Omega_{-}$and $\Omega_{+}$(see Fig. 2). For the present case we choose

$$
u_{\delta}(r, \theta)=\sum_{n=0}^{+\infty} \delta^{n} u_{n}^{+}(r, \theta) \quad \text { in } \Omega_{+}, \quad \text { and } \quad u_{\delta}(r, \theta)=\sum_{n=0}^{+\infty} \delta^{n} u_{n}^{-}(r, \theta) \text { in } \Omega_{-} .
$$

The motivation for choosing such an ansatz comes from the already known asymptotic expansion in the analysis of problems involving a thin layer with a geometry that share similarities with ours, see in particular [30]. We plug (6) into (1) in order to formally derive equations that the terms $u_{n}^{ \pm}$should satisfy. Unfortunately this will not yield a characterization of the $u_{n}^{ \pm}$as these equations will not be well posed: transmission conditions will be missing at the interface $\Gamma$.

Step II: Near field ansatz (Sections 3-4-5): the lack of conditions at the interface will be a motivation for studying the expansion of the field close to the periodic ring. Indeed, in this region, it cannot be expected that an expansion of the form (6) still holds because of the rapidly oscillating structure of the geometry. As a consequence we consider a different form for the asymptotic expansion, namely

$$
\begin{aligned}
& u_{\delta}(r, \theta)=\sum_{n=0}^{+\infty} \delta^{n} U_{n}\left(\frac{r-r_{*}}{\delta}, \frac{r_{*} \theta}{\delta} ; \theta\right), \\
& \text { with } \quad U_{n}(\nu, s+2 k \pi, \tau+2 l \pi)=U_{n}(\nu, s, \tau) \quad \forall k, l \in \mathbb{Z} .
\end{aligned}
$$

Such an ansatz is inspired by the theory of homogenization, see for example [3, 2, 22]. According to the periodicity conditions that we impose on $U_{n}$, it suffices to describe these functions over the infinite periodicity cell $B \times \mathbb{S}^{1}$ represented in figure 2 (b)

$$
B:=\mathbb{R} \times \mathbb{S}^{1} \quad \text { with } \quad \mathbb{S}^{1}=\mathbb{R} / 2 \pi \mathbb{Z} \text { (unit circle of } \mathbb{R}^{2} \text { ). }
$$

Once again we plug the ansatz (7) inside (1) after the change of variables (4). This will yield equations that should be satisfied by the terms $U_{n}(\nu, s, \tau)$, at least formally. However, once again, this set of equations will not be well posed because of a lack of conditions at infinity (for $|\nu| \rightarrow \infty$ ).

Step III: Matching principle (Section 6): in order to obtain well posed problems that would yield a 
characterization of the far and near field terms $u_{n}^{ \pm}, U_{n}$, the equations derived previously will have to be completed with conditions: transmission conditions for $u_{n}^{ \pm}$and conditions at infinity for $U_{n}$. The method of matched asymptotic expansions provides a procedure called "matching principle" for deriving conditions coupling the behavior of $U_{n}(\nu, s, \tau)$ for $|\nu| \rightarrow \infty$ with the behavior of $u_{n}^{ \pm}$close to $\Gamma$. During this step we will apply this procedure.

Step IV: Well-posedness of recurrent problems (Sections 7-8): the final output of Step III above will be a system of recurrent equations. We will show that this system is well posed, so that the terms $u_{n}^{ \pm}, U_{n}$ can be taken as the unique solution to these equations (Theorem 7.1). Semi explicit formula of the far and near field terms are also provided (Theorem 8.3) This step of the analysis will be completely rigorous.

Step V: Error estimate (Section 9): as Step I,II and III of the asymptotic construction partly rely on formal calculus, the purpose of Step V will be an a posteriori justification of the definition of $u_{n}^{ \pm}, U_{n}$ by means of error estimates.

The first main outcome of this analysis will be an explicit recurrent construction of the terms of the expansions (6) and (7) up to any order (see Theorem 7.1 and Problem (59) ). The second outcome of this analysis will be a global error estimate for these expansions (Theorem 10.1), from which we deduce an optimal error estimate for the far field (see Theorem 10.2):

Theorem. Let $\gamma \in\left(0, \gamma_{*}\right)$ and $\mathcal{O}=\left\{\mathbf{x}(r, \theta) \in \Omega|| r-r_{*} \mid>\gamma\right\}$. Then, for any $N \geq 0$, there exists $C_{N}>0$ independent of $\delta$ such that

$$
\left\|u_{\delta}-\sum_{n=0}^{N} \delta^{n} u_{n}\right\|_{\mathrm{H}^{1}(\mathcal{O})} \leq C_{N} \delta^{N+1}
$$

We will provide a completely rigorous justification for this error estimate as well.

Admittedly Step I,II and III of this construction contain a formal part. On the other hand, if we started directly from Step IV which would yield an analysis that is completely rigorous from a mathematical point of view, it would be impossible for the reader to understand where our equations come from. This is what motivated the general structure of the present article.

Notation: periodic functions In the sequel, we will often refer to functions $v(\alpha)$ that are $2 \pi$-periodic with respect to the variable $\alpha=\theta, s$ or $\tau$. This is our motivation for introducing the following space: for any Banach space $\mathbf{V}$ we consider

$$
\mathrm{H}_{\#}^{k}\left(\mathbb{S}^{1}, \mathbf{V}\right)=\left\{v \in \mathrm{H}_{\mathrm{loc}}^{k}(\mathbb{R}, \mathbf{V}) \quad \text { such that } \quad v(\alpha+2 \pi)=v(\alpha)\right\}
$$

This also provides a definition for $C_{\#}^{\infty}\left(\mathbb{S}^{1}, \mathbf{V}\right)=\cap_{k \geq 0} \mathrm{H}_{\#}^{k}\left(\mathbb{S}^{1}, \mathbf{V}\right)$. Note that $\mathrm{H}_{\#}^{k}\left(\mathbb{S}^{1}, \mathbf{V}\right) \subset \mathrm{H}^{k}((0,2 \pi), \mathbf{V})$ but $\mathrm{H}_{\#}^{k}\left(\mathbb{S}^{1}, \mathbf{V}\right) \neq \mathrm{H}^{k}((0,2 \pi), \mathbf{V})$, simply because compatibility conditions relating $v(0), \partial_{\alpha} v(0), \ldots$ to $v(2 \pi), \partial_{\alpha} v(2 \pi), \ldots$ may hold for elements of $\mathrm{H}_{\#}^{k}\left(\mathbb{S}^{1}, \mathbf{V}\right)$.

\section{Far field terms (Step I)}

The first step of our work consists in deriving equations for the far field terms. To do so, we substitute $u_{\delta}$ by its far field expansion (6) in the Helmholtz equation (1) and formally separate the different powers of $\delta$. This yields the equations satisfied by the far field terms: Find $u_{n}^{ \pm} \in \mathrm{H}^{2}\left(\Omega_{ \pm}\right)$such that

$$
\begin{aligned}
& \epsilon_{\infty}^{-1} \triangle u_{n}^{ \pm}+\omega^{2} \mu_{\infty} u_{n}^{ \pm}=f \cdot \delta_{n}^{0} \text { in } \Omega_{ \pm} \\
& \partial_{r} u_{n}^{+}+T u_{n}^{+}=0 \quad \text { on } \partial \Omega .
\end{aligned}
$$


where $\delta_{n}^{0}$ is the kronecker symbol (that has nothing to do with the small parameter $\delta$ ), i.e, $\delta_{0}^{0}=1$ and $\delta_{n}^{0}=0$ for $n \neq 0$. We emphasize that $u_{n}^{ \pm}$are not entirely defined since we have not prescribed yet any boundary condition on $\Gamma$ : we have to find transmission conditions between $u_{n}^{+}$and $u_{n}^{-}$through $\Gamma$. For our asymptotic construction, we impose in addition that

$$
\left.u_{n}\right|_{\Gamma} ^{ \pm} \in C_{\#}^{\infty}\left(\mathbb{S}^{1}\right) \quad \text { and }\left.\quad \partial_{r} u_{n}\right|_{\Gamma} ^{ \pm} \in C_{\#}^{\infty}\left(\mathbb{S}^{1}\right) .
$$

Admittedly (10) is slightly abusive as we should write $C^{\infty}(\Gamma)$ instead of $C_{\#}^{\infty}\left(\mathbb{S}^{1}\right)$. We assume indeed that $\Gamma$ is parameterized by the coordinate $\theta$, so that we consider $\left.u_{n}\right|_{\Gamma} ^{ \pm}$and $\left.\partial_{r} u_{n}\right|_{\Gamma} ^{ \pm}$as periodic functions of $\theta$.

Radial expansion According to classical elliptic regularity results, see for example Theorem 4.18 in [24], $u_{n}^{ \pm}$are smooth in a neighborhood of $\Gamma$, so they admit a radial expansion with respect to $r$ up to any order. More generally, for a function $v \in \mathrm{H}^{2}\left(\Omega_{+}\right)$and $v_{k} \in C^{\infty}\left(\mathbb{S}^{1}\right)$, we shall say that $\sum_{k \geqslant 0}\left(r-r_{*}\right)^{k} v_{k}(\theta)$ is the radial expansion of $v$ for $r-r_{*} \rightarrow 0_{+}$if

$$
\forall n \geq 0, \exists \mathfrak{v}_{n} \in \mathrm{H}^{2}\left(\Omega_{+}\right) \quad \text { s.t. } \quad v(r, \theta)-\sum_{k=0}^{n}\left(r-r_{*}\right)^{k} v_{k}(\theta)=\left(r-r_{*}\right)^{n+1} \mathfrak{v}_{n}(r, \theta) .
$$

Decomposition of the Helmholtz operator We would like to describe in detail the terms of the radial expansion of $u_{n}^{ \pm}(r, \theta)$. To do so we introduce a particular decomposition of the Helmholtz operator. Our approach is an application of a more general method proposed in [10]. Using the expression of the Laplace operator in the polar coordinates, we can decompose the operator $r^{2}\left(\epsilon_{\infty}^{-1} \Delta+\omega^{2} \mu_{\infty}\right)$ according to $\rho=r-r_{*}$

$$
\mathscr{A} u=r^{2}\left(\epsilon_{\infty}^{-1} \Delta+\omega^{2} \mu_{\infty}\right) u=\frac{1}{\rho^{2}} \sum_{j=0}^{4} \rho^{j} \mathscr{A}_{j}\left(\rho \partial_{\rho}, \partial_{\theta}\right) u,
$$

where $\mathscr{A}_{j}\left(\rho \partial_{\rho}, \partial_{\theta}\right),(j=0 \cdots 4)$, are given by

$$
\begin{aligned}
& \mathscr{A}_{0}\left(\rho \partial_{\rho}, \partial_{\theta}\right) u=r_{*}^{2} \epsilon_{\infty}^{-1}\left[\left(\rho \partial_{\rho}\right)^{2}-\rho \partial_{\rho}\right] u \\
& \mathscr{A}_{1}\left(\rho \partial_{\rho}, \partial_{\theta}\right) u=r_{*} \epsilon_{\infty}^{-1}\left[2\left(\rho \partial_{\rho}\right)^{2}-\rho \partial_{\rho}\right] u \\
& \mathscr{A}_{2}\left(\rho \partial_{\rho}, \partial_{\theta}\right) u=\left[\epsilon_{\infty}^{-1}\left(\rho \partial_{\rho}\right)^{2}+\epsilon_{\infty}^{-1} \partial_{\theta}^{2}+\mu_{\infty} \omega^{2} r_{*}^{2}\right] u \\
& \mathscr{A}_{3}\left(\rho \partial_{\rho}, \partial_{\theta}\right) u=2 \omega^{2} \mu_{\infty} r_{*} u \\
& \mathscr{A}_{4}\left(\rho \partial_{\rho}, \partial_{\theta}\right) u=\omega^{2} \mu_{\infty} u
\end{aligned}
$$

The operators $\mathscr{A}_{j}$ satisfy some kind of homogeneity property: for any $u(r, \theta)=\left(r-r_{*}\right)^{k} u_{k}(\theta)=\rho^{k} u_{k}(\theta)$, there exists a function $v_{k}(\theta)$ that only depends on $\theta$ such that $\mathscr{A}_{j} u=\left(r-r_{*}\right)^{k} v_{k}(\theta)$. This remark leads us to introduce, for all $k \in \mathbb{R}$, the operators $\mathscr{A}_{j}(k)$ :

$$
\mathscr{A}_{j}\left(k, \partial_{\theta}\right) \cdot v=\rho^{-k} \mathscr{A}_{j}\left(\rho \partial_{\rho}, \partial_{\theta}\right) \cdot\left\{\rho^{k} v(\theta)\right\}
$$

The $\mathscr{A}_{j}\left(k, \partial_{\theta}\right)$ are differential operators with respect to $\theta$. We shall see that $\mathscr{A}_{0}\left(k, \partial_{\theta}\right)$ plays a particular role in the forthcoming analysis. Note that $\mathscr{A}_{0}\left(k, \partial_{\theta}\right)=\mathscr{A}_{0}(k)=r_{*}^{2} \epsilon_{\infty}^{-1}\left(k^{2}-k\right)$ so, as a simple number, $\mathscr{A}_{0}(k)$ is invertible except for $k=0,1$ and $\mathscr{A}_{0}(k)^{-1} v(\theta)=v(\theta) /\left[r_{*}^{2} \epsilon_{\infty}^{-1}\left(k^{2}-k\right)\right]$.

Far field behavior close to the interface In this paragraph we will describe the general form of the asymptotics of solutions to the Helmholtz equation in the vicinity of the interface $\Gamma$. We consider here that $r>r_{*}$, but the same analysis could be carried out mutatis mutandis for $r<r_{*}$ taking the same notations.

Assume that $v \in \mathrm{H}^{2}\left(\Omega_{+}\right)$satisfies $\epsilon_{\infty}^{-1} \Delta v+\omega^{2} \mu_{\infty} v=0$ in a neighborhood of the interface $\Gamma$, and that it admits a radial expansion of the form (11) up to any order. What does Helmholtz Equation imply on 
the terms $v_{k}(\theta)$ of its asymptotic series? Inserting this series in the decomposition (12) of the operator $\mathscr{A}$ yields $\sum_{k=0}^{4} \sum_{j \in \mathbb{N}} \rho^{k} \mathscr{A}_{k}\left(\rho^{j} v_{j}\right)=0$ which yields, after reordering,

$$
\sum_{k \geqslant 0} \rho^{k}\left\{\sum_{j=0}^{4} \mathscr{A}_{j}\left(k-j, \partial_{\theta}\right) \cdot v_{k-j}(\theta)\right\}=0 \Longrightarrow \sum_{j=0}^{4} \mathscr{A}_{j}\left(k-j, \partial_{\theta}\right) \cdot v_{k-j}(\theta)=0, \quad \forall k \geqslant 0,
$$

where we take the convention that $v_{j}=0$ for $j<0$. The equations above yield an iterative process for determining $v_{k}(\theta), k \geqslant 2$ provided that $v_{0}(\theta), v_{1}(\theta)$ are already known: this is a straightforward consequence of

$$
v_{k}(\theta)=-\mathscr{A}_{0}(k)^{-1} \sum_{j=1}^{4} \mathscr{A}_{j}\left(k-j, \partial_{\theta}\right) \cdot v_{k-j}(\theta), \quad \forall k \geqslant 2 .
$$

that makes sense only for $j \geqslant 2$ since $\mathscr{A}_{0}(0)=\mathscr{A}_{0}(1)=0$. In this construction process, the first two terms of the asymptotics $v_{0}(\theta), v_{1}(\theta)$ play the role of initial conditions. Note that they are simply the Dirichlet and Neumann trace of $v$,

$$
v_{0}(\theta)=\left.v\right|_{\Gamma} ^{+} \quad \text { and } \quad v_{1}(\theta)=\left.\partial_{r} v\right|_{\Gamma} ^{+} .
$$

As a consequence, if $\epsilon_{\infty} \Delta v+\omega^{2} \mu_{\infty} v=0$ in the vicinity of $\Gamma$ and $\left.v\right|_{\Gamma} ^{+}$and $\left.\partial_{r} v\right|_{\Gamma} ^{+}$are known, then the whole expansion of $v$ can be explicitly constructed by means of (15)-(16). This conclusion can be formalized as follows. Introduce two families of differential operators with respect to $\theta$ that are denoted $\left(s_{k}^{0}\left(\partial_{\theta}\right)\right)_{k \geq 0}$ and $\left(s_{k}^{1}\left(\partial_{\theta}\right)\right)_{k \geq 0}$. These operators are defined by an iterative procedure that mimics the construction above.

$$
\begin{aligned}
& s_{k}^{0}\left(\partial_{\theta}\right)=0 \quad \forall k<0, \quad s_{0}^{0}\left(\partial_{\theta}\right)=\mathrm{Id}, \quad s_{1}^{0}=0, \\
& s_{k}^{0}\left(\partial_{\theta}\right)=-\mathscr{A}_{0}(k)^{-1} \sum_{j=1}^{4} \mathscr{A}_{j}\left(k-j, \partial_{\theta}\right) \cdot s_{k-j}^{0}\left(\partial_{\theta}\right) \quad k \geq 2, \\
& s_{k}^{1}\left(\partial_{\theta}\right)=0 \quad \forall k<0, \quad s_{0}^{1}\left(\partial_{\theta}\right)=0, \quad s_{1}^{1}\left(\partial_{\theta}\right)=\mathrm{Id}, \\
& s_{k}^{1}\left(\partial_{\theta}\right)=-\mathscr{A}_{0}(k)^{-1} \sum_{j=1}^{4} \mathscr{A}_{j}\left(k-j, \partial_{\theta}\right) \cdot s_{k-j}^{1}\left(\partial_{\theta}\right) \quad k \geq 2 .
\end{aligned}
$$

\section{Proposition 2.1.}

Let $v \in \mathrm{H}^{2}\left(\Omega_{+}\right)$satisfy $\epsilon_{\infty}^{-1} \Delta v+\omega^{2} \mu_{\infty} v=0$ in the vicinity of the interface $\Gamma$, and assume that it admits a radial expansion up to any order. Then $\forall n \geq 0$ there exists $\mathfrak{v}_{n} \in \mathrm{H}^{2}\left(\Omega_{+}\right)$such that

$$
v(r, \theta)=\sum_{k=0}^{n}\left(r-r_{*}\right)^{k}\left(\left.s_{k}^{0}\left(\partial_{\theta}\right) v\right|_{\Gamma} ^{+}+\left.s_{k}^{1}\left(\partial_{\theta}\right) \partial_{r} v\right|_{\Gamma} ^{+}\right)+\left(r-r_{*}\right)^{n+1} \mathfrak{v}_{n}(r, \theta) \quad \forall n \geq 0 .
$$

Proof. By assumption we have $v(r, \theta)=\sum_{k>0}\left(r-r_{*}\right)^{k} v_{k}(\theta)$. To prove (18), we prove that $v_{k}(\theta)=$ $s_{k}^{0}(\theta) v_{0}(\theta)+s_{k}^{1}\left(\partial_{\theta}\right) v_{1}(\theta)$ by induction. This is immediate for $k=0$ and $k=1$. Now assume that $v_{j}(\theta)=s_{j}^{0}\left(\partial_{\theta}\right) v_{0}(\theta)+s_{j}^{1}\left(\partial_{\theta}\right) v_{1}(\theta)$ holds for any $j \leq k$. Then according to (15) and (17) we have

$$
\begin{aligned}
v_{k+1}(\theta) & =-\mathscr{A}_{0}(k+1)^{-1} \sum_{j=1}^{4} \mathscr{A}_{j}\left(k+1-j, \partial_{\theta}\right) \cdot\left(s_{k+1-j}^{0}\left(\partial_{\theta}\right) v_{0}(\theta)+s_{k+1-j}^{1}\left(\partial_{\theta}\right) v_{1}(\theta)\right), \\
& =s_{k+1}^{0}\left(\partial_{\theta}\right) v_{0}(\theta)+s_{k+1}^{1}\left(\partial_{\theta}\right) v_{1}(\theta) .
\end{aligned}
$$

Proposition 2.1 also holds for functions defined in $\Omega_{-}$. Since we impose Equations (9) and (10), Proposition 2.1 can be applied to the far field terms: $\forall n, p \geq 0$, there exists $\mathfrak{u}_{n, p} \in \mathrm{L}^{2}(\Omega)$ such that $\left.\mathfrak{u}_{n, p}\right|_{\Omega_{ \pm}} \in$ $\mathrm{H}^{2}\left(\Omega_{ \pm}\right)$and that satisfies

$$
u_{n}^{ \pm}(r, \theta)=\sum_{k=0}^{p}\left(r-r_{*}\right)^{k}\left(\left.s_{k}^{0}\left(\partial_{\theta}\right) u_{n}\right|_{\Gamma} ^{ \pm}+\left.s_{k}^{1}\left(\partial_{\theta}\right) \partial_{r} u_{n}\right|_{\Gamma} ^{ \pm}\right)+\left(r-r_{*}\right)^{p+1} \mathfrak{u}_{n, p}(r, \theta) .
$$


Notation: mean and jump operators For the sake of brevity, from now on, we will write $s_{j}^{0}\{v\}$ and $s_{j}^{1}\{v\}$ instead of $s_{j}^{0}\left(\partial_{\theta}\right) v$ and $s_{j}^{1}\left(\partial_{\theta}\right) v$. In the sequel we will need notations for Dirichlet and Neumann jump and mean values on $\Gamma$ for a function $v \in \mathrm{H}^{1}\left(\Omega_{ \pm}\right)$such that $\Delta v \in \mathrm{L}^{2}\left(\Omega_{ \pm}\right)$so we set

$$
\begin{aligned}
{[v]_{\Gamma} } & =\left.v\right|_{\Gamma} ^{+}-\left.v\right|_{\Gamma} ^{-}, \quad\left[\partial_{r} v\right]_{\Gamma}=\left.\partial_{r} v\right|_{\Gamma} ^{+}-\left.\partial_{r} v\right|_{\Gamma} ^{-} \\
\text {and } \quad\langle v\rangle_{\Gamma} & =\frac{\left.v\right|_{\Gamma} ^{+}+\left.v\right|_{\Gamma} ^{-}}{2}, \quad\left\langle\partial_{r} v\right\rangle_{\Gamma}=\frac{\left.\partial_{r} v\right|_{\Gamma} ^{+}+\left.\partial_{r} v\right|_{\Gamma} ^{-}}{2} .
\end{aligned}
$$

\section{Near field terms (Step II)}

This paragraph is dedicated to the derivation of equations that should be satisfied by the terms of the near field expansion. This will be a much more involved task than for the far field terms: these issues were predictable because of the scaling appearing in (7). Let us rewrite (7) as follows:

$$
u_{\delta}(r, \theta)=\sum_{n=0}^{\infty} \delta^{n} U_{n}^{\delta}(r, \theta) \quad \text { where } \quad U_{n}^{\delta}(r, \theta):=U_{n}\left(\frac{r-r_{*}}{\delta}, \frac{r_{*} \theta}{\delta}, \theta\right) \quad \forall n \in \mathbb{N} .
$$

In this ansatz the terms $U_{n}$ are functions of variables denoted $(\nu, s, \tau)$. Although the identity $\tau=\theta$ will always hold throughout our analysis, it seemed to us that distinguishing both variables would help remove any ambiguity in the equations.

\subsection{Derivation of the equations satisfied by the near field terms}

In this paragraph, we will formally derive the equations that the terms of the expansion (20) should satisfy. We shall use the formulas

$$
\begin{aligned}
& \frac{\partial U_{n}^{\delta}}{\partial r}(r, \theta)=\frac{1}{\delta} \frac{\partial U_{n}}{\partial \nu}\left(\frac{r-r_{*}}{\delta}, \frac{r_{*} \theta}{\delta} ; \theta\right), \\
& \frac{\partial U_{n}^{\delta}}{\partial \theta}(r, \theta)=\frac{r_{*}}{\delta} \frac{\partial U_{n}}{\partial s}\left(\frac{r-r_{*}}{\delta}, \frac{r_{*} \theta}{\delta} ; \theta\right)+\frac{\partial U_{n}}{\partial \tau}\left(\frac{r-r_{*}}{\delta}, \frac{r_{*} \theta}{\delta} ; \theta\right) .
\end{aligned}
$$

Using the expression of the laplacian in polar coordinates and replacing $r$ by $r_{*}+\delta \nu$ and taking into account that $\epsilon^{-1}$ does not depend on $\theta$, easy calculation yields

$$
\begin{aligned}
& r^{2}\left(\operatorname{div}\left(\epsilon_{\delta}^{-1} \nabla U_{n}^{\delta}\right)+\mu_{\delta} \omega^{2} U_{n}^{\delta}\right)= \\
& \quad \frac{1}{\delta^{2}} r_{*}^{2}\left[\partial_{\nu}\left(\epsilon^{-1} \partial_{\nu} U_{n}\right)+\partial_{s}\left(\epsilon^{-1} \partial_{s} U_{n}\right)\right] \\
&+\frac{1}{\delta} r_{*}\left[2 \nu \partial_{\nu}\left(\epsilon^{-1} \partial_{\nu} U_{n}\right)+\epsilon^{-1} \partial_{\nu} U_{n}+\partial_{s}\left(\epsilon^{-1} \partial_{\tau} U_{n}\right)+\partial_{\tau}\left(\epsilon^{-1} \partial_{s} U_{n}\right)\right] \\
&+\quad \nu \partial_{\nu}\left(\epsilon^{-1} \nu \partial_{\nu} U_{n}\right)+\epsilon^{-1} \partial_{\tau}^{2} U_{n}+r_{*}^{2} \mu \omega^{2} U_{n} \\
&+\delta 2 \nu r_{*} \mu \omega^{2} U_{n} \\
&+ \delta^{2} \nu^{2} \mu \omega^{2} U_{n} .
\end{aligned}
$$

Since $u_{\delta}$ solves the homogeneous Helmholtz equation in the vicinity of the periodic ring, we have formally $\sum_{n \in \mathbb{N}} \delta^{n} r^{2}\left(\operatorname{div}\left(\epsilon_{\delta}^{-1} \nabla U_{n}^{\delta}\right)+\mu_{\delta} \omega^{2} U_{n}^{\delta}\right)=0$. Plugging (22) in this equation, collecting the terms in $\delta^{n}$, and setting as a convention that $U_{n}=0$ for $n \leq 0$, we obtain the following equations

$$
\nu^{-2} A_{0}\left(\partial_{\nu}, \partial_{s}\right) U_{n}=-\nu^{-2} \sum_{j=1}^{4} \nu^{j} A_{j} U_{n-j} \quad \text { in } B \times \mathbb{S}^{1} .
$$


Admittedly, we should impose the equations of (23) only for $s=r_{*} \tau / \delta$. However following a typical homogeneization methodology, we deliberately choose to "relax" the constraint $s=r_{*} \tau / \delta$ and to impose the equations of (23) for any $s$ and $\tau$, which is stronger. This choice will be justified a posteriori by the error estimate of Section 10. The differential operator $A_{0}=A_{0}\left(\partial_{\nu}, \partial_{s}\right)$ is defined by

$$
A_{0}\left(\partial_{\nu}, \partial_{s}\right) U:=r_{*}^{2} \nu^{2}\left(\partial_{\nu}\left(\epsilon^{-1} \partial_{\nu} U\right)+\partial_{s}\left(\epsilon^{-1} \partial_{s} U\right)\right)
$$

Observe that $A_{0}$ does not contain any dependency nor any partial derivative with respect to $\tau$ which will be a key feature later on. The other operators $A_{j}, 1 \leq j \leq 4$ are differential operators in $(\nu, s, \tau)$ defined by

$$
\begin{aligned}
& A_{1} U:=2 \nu^{2} \partial_{\nu}\left(\epsilon^{-1} \partial_{\nu} U\right)+\epsilon^{-1} \nu \partial_{\nu} U+\nu \partial_{s}\left(\epsilon^{-1} \partial_{\tau} U\right)+\nu \partial_{\tau}\left(\epsilon^{-1} \partial_{s} U\right) \\
& A_{2} U:=\nu \partial_{\nu}\left(\epsilon^{-1} \nu \partial_{\nu} U\right)+\epsilon^{-1} \partial_{\tau}^{2} U+r_{*}^{2} \mu \omega^{2} U \\
& A_{3} U:=2 \omega^{2} \mu r_{*} U \\
& A_{4} U:=\omega^{2} \mu U .
\end{aligned}
$$

Note that, according to these definitions, for any function $U(\nu, s, \tau)=U(\nu, \tau)$ that does not depend on the variable $s$ we have $A_{k}\left(\partial_{\nu}, \partial_{s}, \partial_{\tau}\right) U(\nu, \tau)=\mathscr{A}_{k}\left(\nu \partial_{\nu}, \partial_{\tau}\right) U(\nu, \tau)$ for $k=0, \ldots, 4$ and $|\nu|>2 \pi$, where $\mathscr{A}_{k}\left(\nu \partial_{\nu}, \partial_{\tau}\right)$ has been defined in (13).

\subsection{Precise statement of the near field equations}

The material of subsection 3.1 was formal. In the present paragraph we will give a rigorous mathematical sense to Equations (23). We need to introduce an adapted functional framework. First of all, we will assume that the functions $U_{n}$ admit a smooth dependency with respect to $\tau$ and that $U_{n}(\nu, s, \tau+2 \pi)=U_{n}(\nu, s, \tau)$.

\subsubsection{Weighted spaces}

In accordance with our choice of ansatz for the near field, we also have to impose the periodicity condition $U_{n}(\nu, s+2 \pi, \tau)=U_{n}(\nu, s, \tau)$. Moreover, following the usual procedure of matched asymptotics, we also have to discard any possibility for the terms $U_{n}$ to blow up exponentially for $|\nu| \rightarrow \infty$, which can be justified a posteriori. In the present case, this condition will be enforced by imposing that, for any $n$ and any $\theta$, the function $U_{n}(\cdot, \tau) \in \mathbb{V}_{+}^{1}(B)$ where we set, for $\sigma= \pm$,

$$
\begin{array}{r}
\mathbb{V}_{\sigma}^{k}(B)=\left\{\left.U \in \mathrm{H}_{\mathrm{loc}}^{k}\left(\mathbb{R}^{2}\right)\left|\|U\|_{\mathbb{V}_{\sigma}^{k}}^{2}=\sum_{\alpha+\beta \leq k} \int_{B}\right| \partial_{\nu}^{\alpha} \partial_{s}^{\beta} U(\nu, s)\right|^{2} e^{-\sigma|\nu|} d \nu d s<+\infty\right. \\
\text { and } U(\nu, s+2 \pi)=U(\nu, s)\} .
\end{array}
$$

We emphasize that the definition of $\mathbb{V}_{\sigma}^{k}(B)$ encompasses a periodicity assumption with respect to $s$. We will use the above definition for $k=0$ and $k=1$. The spaces $\mathbb{V}_{ \pm}^{k}(B)$ equipped with the norm \|\|$_{\mathbb{V}_{ \pm}^{k}}$ are Banach spaces. Besides we have the obvious inclusion $\mathbb{V}_{-}^{k}(B) \subset \mathbb{V}_{+}^{k}(B)$. The elements of $\mathbb{V}_{-}^{1}(B)$ may be interpreted as "evanescent at infinity". Finally we impose that the functions $U_{n}$ should belong to the following space

$$
U_{n} \in C^{\infty}\left(\mathbb{S}^{1}, \mathbb{V}_{+}^{1}(B)\right) \quad \forall n \in \mathbb{Z}
$$

\subsubsection{Dual spaces}

In the sequel, we may write equations in the space $\mathbb{V}_{+}^{1}(B)^{\prime}$ the topological dual space of $\mathbb{V}_{+}^{1}(B)$ i.e. the space of linear functionals that are continuous over $\mathbb{V}_{+}^{1}$ for the norm \|\|$_{\mathbb{V}_{+}^{1}}$. The duality pairing on $\left(\mathbb{V}_{+}^{1}\right)^{\prime} \times \mathbb{V}_{+}^{1}$ will be denoted $<;>_{+}$. As usual, we equip this space with the continuity norm associated to this pairing,

$$
\|g\|_{\left(\mathbb{V}_{+}^{1}\right)^{\prime}}=\sup _{U \in \mathbb{V}_{+}^{1} \backslash\{0\}} \frac{\langle g, U\rangle_{+}}{\|U\|_{\mathbb{V}_{+}^{1}}} .
$$


The following lemma gives a detailed description of $\mathbb{V}_{+}^{1}(B)^{\prime}$ by showing that it can be identified with a space of distributions over $\mathbb{R}^{2}$ that are periodic in $s$.

\section{Lemma 3.1.}

$\operatorname{Set}\left(T_{\#} \varphi\right)(\nu, s)=\sum_{k \in \mathbb{Z}} \varphi(\nu, s+2 k \pi), \forall \varphi \in \mathscr{D}\left(\mathbb{R}^{2}\right)$. For any $g \in \mathbb{V}_{+}^{1}(B)^{\prime}$ set $T_{\#}^{\star} g: \varphi \mapsto\left\langle g, T_{\#} \varphi\right\rangle_{+}$ that is an element of $\mathscr{D}^{\prime}\left(\mathbb{R}^{2}\right)$. The image of $\mathbb{V}_{+}^{1}(B)^{\prime}$ under $T_{\#}^{\star}$ is exactly the space of distributions $h \in$ $\mathscr{D}^{\prime}\left(\mathbb{R}^{2}\right)$ of the form $h=h_{1}-\operatorname{div}\left(h_{2}\right)$ where $h_{1}, h_{2} \in \mathrm{L}_{\text {loc }}^{2}\left(\mathbb{R}^{2}\right)$ such that $h_{k}(\nu, s+2 l \pi)=h_{k}(\nu, s), \forall s \in$ $\mathbb{R}, \forall l \in \mathbb{Z}$ and $\left.h_{k}\right|_{B} \in \mathbb{V}_{-}^{0}(B)$ for $k=1,2$.

Remark 3.2. Assume that $g \in \mathbb{V}_{+}^{1}(B)^{\prime}$ is in $C_{\#}^{\infty}(B)$. Then, $\left(T_{\#}^{\star} g\right)$ is the $2 \pi$ periodic function (in $s$ ) which satisfies $\left(T_{\#}^{\star} g\right)_{\mid B}=g$. In other words $T_{\#}^{\star} g$ is the extension of $g$ by periodicity. Note also that one practical interest of the previous notation is the justification of the Green formula (28).

Proof. Take a $g \in \mathbb{V}_{+}^{1}(B)^{\prime}$. Observe that $(u, v) \mapsto \int_{B}(\nabla u \cdot \nabla \bar{v}+u \bar{v}) e^{-|\nu|} d \nu d s$ is a scalar product over $\mathbb{V}_{+}^{1}(B)$. As a consequence according to Riesz representation theorem, there exists $u_{g} \in \mathbb{V}_{+}^{1}(B)$ such that

$$
\langle g, v\rangle_{+}=\int_{B}\left(e^{-|\nu|} \nabla u_{g}\right) \cdot \nabla \bar{v}+\left(e^{-|\nu|} u_{g}\right) \bar{v} d \nu d s \quad \forall v \in \mathbb{V}_{+}^{1}(B) .
$$

Set $g_{1}=e^{-|\nu|} u_{g}$ and $g_{2}=e^{-|\nu|} \nabla u_{g}$, and observe that $g_{k} \in \mathbb{V}_{-}^{0}(B)$. For $k=1,2$ consider $h_{k} \in \mathrm{L}_{l o c}^{2}\left(\mathbb{R}^{2}\right)$ such that $h_{k}(\nu, s+2 l \pi), \forall l \in \mathbb{Z}$ and $\left.h_{k}\right|_{B}=g_{k}$. Observe that $\int_{B} g_{1} T_{\#} \varphi d \mathbf{x}=\int_{\mathbb{R}^{2}} h_{1} \varphi d \mathbf{x}, \forall \varphi \in \mathscr{D}\left(\mathbb{R}^{2}\right)$ and the same remark holds for $g_{2}, h_{2}$. As a consequence $T_{\#}^{\star} g=h_{1}-\operatorname{div}\left(h_{2}\right)$ since

$$
\begin{aligned}
\left\langle T_{\#}^{\star} g, \varphi\right\rangle_{\mathscr{D}^{\prime}, \mathscr{D}} & =\int_{B} g_{1} T_{\#} \varphi d \nu d s+\int_{B} g_{2} \cdot \nabla\left(T_{\#} \varphi\right) d \nu d s \\
& =\int_{B} g_{1} T_{\#} \varphi d \nu d s+\int_{B} g_{2} \cdot T_{\#}(\nabla \varphi) d \nu d s \\
& =\int_{\mathbb{R}^{2}} h_{1} \varphi d \nu d s+\int_{\mathbb{R}^{2}} h_{2} \cdot \nabla \varphi d \nu d s \quad \forall \varphi \in \mathscr{D}\left(\mathbb{R}^{2}\right)
\end{aligned}
$$

where $<\cdot, \cdot>\mathscr{D}^{\prime}, \mathscr{D}$ denotes the duality product between $\mathscr{D}^{\prime}\left(\mathbb{R}^{2}\right)$ and $\mathscr{D}\left(\mathbb{R}^{2}\right)$. Conversely consider $h \in$ $\mathscr{D}^{\prime}\left(\mathbb{R}^{2}\right)$ of the form $h=h_{1}-\operatorname{div}\left(h_{2}\right)$ where $h_{1}, h_{2}$ satisfy the conditions stated in the lemma. Consider $g_{k}=\left.h_{k}\right|_{B}, k=1,2$ and define $g \in \mathbb{V}_{+}^{1}(B)^{\prime}$ by

$$
\langle g, \varphi\rangle_{+}=\left\langle g_{1}, v\right\rangle_{+}-\left\langle\operatorname{div}\left(g_{2}\right), v\right\rangle_{+}=\int_{B} g_{1} \varphi+g_{2} \cdot \nabla \varphi d \nu d s .
$$

A calculus similar to what precedes shows that $T_{\#}^{\star} g=h$.

Convention of notation The elements of $\mathbb{V}_{+}^{1}(B)^{\prime}$ can be interpreted as periodic distributions of $\mathscr{D}^{\prime}\left(\mathbb{R}^{2}\right)$. As a consequence, whenever $h \in \mathscr{D}^{\prime}\left(\mathbb{R}^{2}\right)$ we shall adopt the following convention of notation

$$
" g=h \text { in } \mathbb{V}_{+}^{1}(B)^{\prime} " \quad \Longleftrightarrow \quad " g \in \mathbb{V}_{+}^{1}(B)^{\prime} \text { and } T_{\#}^{\star} g=h \text { in } \mathscr{D}^{\prime}\left(\mathbb{R}^{2}\right) " .
$$

For a distribution $h$ belonging a priori to $\mathscr{D}^{\prime}\left(\mathbb{R}^{2}\right)$ we shall also write $h \in \mathbb{V}_{+}^{1}(B)^{\prime}$ which would mean precisely: " $\exists g \in \mathbb{V}_{+}^{1}(B)^{\prime}$ such that $h=T_{\#} g$ in $\mathscr{D}^{\prime}\left(\mathbb{R}^{2}\right)^{\prime}$.

Concerning $\mathbb{V}_{-}^{1}(B)^{\prime}$, a lemma similar to Lemma 3.1 holds, so we set mutatis mutandis the same conventions of notation for $\mathbb{V}_{-}^{1}(B)^{\prime}$. Besides $\mathbb{V}_{+}^{1}(B)^{\prime} \subset \mathbb{V}_{-}^{1}(B)^{\prime}$ since $\mathbb{V}_{-}^{1}(B) \subset \mathbb{V}_{+}^{1}(B)$. And indeed careful verifications show that, for $h \in \mathscr{D}^{\prime}\left(\mathbb{R}^{2}\right)$, we have

$$
" g=h \text { in } \mathbb{V}_{+}^{1}(B)^{\prime} " \Longrightarrow " g=h \text { in } \mathbb{V}_{-}^{1}(B)^{\prime} " .
$$




\subsubsection{Equations of the near field}

Now we come back to the equations that we collected previously for the near field terms, imposing smooth dependency with respect to $\tau$ and discarding any exponential growth for $|\nu| \rightarrow \infty$. We obtain the following recurrent system of equations, where $U_{n}$ is the unknown and $U_{n-1}, U_{n-2}, U_{n-3}$ and $U_{n-4}$ are considered already known

$$
\begin{aligned}
& U_{n} \in C^{\infty}\left(\mathbb{S}^{1}, \mathbb{V}_{+}^{1}(B)\right) \\
& A_{0}\left(\partial_{\nu}, \partial_{s}\right) U_{n}=-\sum_{j=1}^{4} \nu^{j} A_{j}\left(\partial_{\nu}, \partial_{s}, \partial_{\tau}\right) U_{n-j} \quad \text { in } \mathbb{V}_{-}^{1}(B)^{\prime}, \quad \forall \tau \in \mathbb{S}^{1}
\end{aligned}
$$

In these equations the operators $A_{j}, j=1 \ldots 4$ involve $\partial_{\nu}, \partial_{s}, \partial_{\tau}$ and admit some dependency with respect to $\nu$ and $s$, as can be checked from (25). Besides note that writing equations $(30)$ in $\mathbb{V}_{-}^{1}(B)^{\prime}$ allows the right hand side of the equation to admit a polynomial growth for $|\nu| \rightarrow \infty$. We will use this system of equations for defining each $U_{n}$. However (30) is not well posed as the conditions at infinity are not constraining enough.

\section{Study of the Laplace equation in the normalized strip}

The equations that we have just derived for the terms of the near field asymptotics raise some difficulty at least for two reasons. First of all, (30) has a recurrent structure. In addition, there is no particular reason for assuming that the right-hand side would remain bounded at infinity. As a consequence, (30) appears as a complicated problem that does not fit any particular standard framework.

Consequently, we stop for a while our asymptotic procedure, and we dedicate the present section to the study of equations that take the same form as (30). We will also describe precisely the behavior at infinity of the solutions to such equations and we will give important results of well-posedness (Propositions 4.5 4.7 and 4.8) . Note that the present section only contains completely rigorous analysis.

In this paragraph, we only consider functions of $\nu, s$, and $(\nu, s)$ will be thought as cartesian coordinates over $\mathbb{R}^{2}$, so that the operator $\nu^{-2} A_{0}$ then simply rewrites $\nu^{-2} A_{0} \cdot U=r_{*}^{2} \operatorname{div}\left(\epsilon^{-1} \nabla U\right)$. Suppose given $g \in \mathbb{V}_{+}^{1}(B)^{\prime}$ and consider the problem

$$
\begin{aligned}
& \text { Find } U \in \mathbb{V}_{+}^{1}(B) \quad \text { such that } \\
& -\operatorname{div}\left(\epsilon^{-1} \nabla U\right)=g \quad \text { in } \mathbb{V}_{+}^{1}(B)^{\prime} .
\end{aligned}
$$

Here the left hand side $\operatorname{div}\left(\epsilon^{-1} \nabla U\right)$ makes sense as an element of $\mathscr{D}^{\prime}\left(\mathbb{R}^{2}\right)$ so that (31) should be understood in the sense of (29). Let $\mathscr{D}_{\#}(B)$ refer to the space of functions $\varphi \in C^{\infty}\left(\mathbb{R}^{2}\right)$ such that $\varphi(\nu, s+2 k \pi)=$ $\varphi(\nu, s) \forall k \in \mathbb{Z}, \forall \nu, s \in \mathbb{R}$ and there exists $\nu_{0}>0$ such that $\varphi(\nu, s)=0$ for $|\nu|>\nu_{0}$. In particular we have $T_{\#}\left(\mathscr{D}\left(\mathbb{R}^{2}\right)\right)=\mathscr{D}_{\#}(B)$ according to Lemma 11.2 so that Equation (31) can be reformulated variationally as follows

$$
\int_{B} \epsilon^{-1} \nabla U \cdot \nabla \varphi d \mathbf{x}=\langle g, \varphi\rangle_{+} \quad \forall \varphi \in \mathscr{D}_{\#}(B) .
$$

\subsection{Standard result of well-posedness : variational framework}

According to standard techniques for the Laplace equation, existence of a solution to 31 is at hand in a slightly different functional framework. Indeed, it is natural to rely on a variational approach in order to determine whether this problem is well posed. As a variational framework, we consider the following spaces

$$
\mathbb{W}(B)=\left\{\left.V \in \mathrm{H}_{\mathrm{loc}}^{1}\left(\mathbb{R}^{2}\right)\left|\int_{B}\right| \nabla V\right|^{2}+\frac{|V|^{2}}{1+\nu^{2}} d \nu d s<\infty \text { and } V(\nu, s+2 \pi)=V(\nu, s)\right\} .
$$


Note that we have the inclusions $\mathbb{V}_{-}^{1}(B) \subset \mathbb{W}(B) \subset \mathbb{V}_{+}^{1}(B)$. This implies in particular $\mathbb{V}_{+}^{1}(B)^{\prime} \subset \mathbb{W}(B)^{\prime}$ where $\mathbb{W}(B)^{\prime}$ is the topological dual to the space $\mathbb{W}(B)$. Observe that $1 \in \mathbb{W}(B)$ and $\nu \notin \mathbb{W}(B)$. Besides a Poincaré inequality holds for this space. Indeed set $\Gamma_{ \pm}:=\{ \pm \pi\} \times(0,2 \pi)$. Then there exists a constant $C>0$ such that, for any $U \in \mathbb{W}(B)$, we have

$$
\|U\|_{\mathbb{W}}^{2}=\int_{B}|\nabla U|^{2}+\frac{|U|^{2}}{1+\nu^{2}} d \nu d s \leq C \int_{B}|\nabla U|^{2} d \nu d s+C\left|\int_{\Gamma_{-}} U d s+\int_{\Gamma_{+}} U d s\right|^{2} .
$$

This is a consequence of Hardy's inequality, see for instance Lemma 2.5.7 in [25]. The next proposition is a byproduct of this inequality. The proof follows directly from Lax-Milgram's Lemma.

\section{Proposition 4.1.}

Let $g \in \mathbb{V}_{+}^{1}(B)^{\prime}$. Then there exists at most one function $U \in \mathbb{W}(B)$ satisfying both $-\operatorname{div}\left(\epsilon^{-1} \nabla U\right)=g$ in $\mathbb{V}_{+}^{1}(B)^{\prime}$ and the condition

$$
\int_{\Gamma_{-}} U d s+\int_{\Gamma_{+}} U d s=0 \quad \text { with } \quad \Gamma_{ \pm}:=\{ \pm \pi\} \times(0,2 \pi) .
$$

This solution exists if and only if the following compatibility condition is satisfied: $\langle g, 1\rangle_{+}=0$. Besides, there is continuous dependency of $U$ with respect to $g$ i.e. there exists a constant $C>0$ independent of $g$ such that

$$
\|U\|_{\mathbb{W}} \leqslant C\|g\|_{\left(\mathbb{V}_{+}^{1}\right)^{\prime}}
$$

The only technical point for proving Proposition 4.1 consists in establishing that 32 still holds with $\varphi$ chosen arbitrarily in $\mathbb{W}(B)$. But this is a consequence of the density of $\mathscr{D}_{\#}(B)$ in $\mathbb{W}(B)$ for the norm $\|\cdot\|_{\mathbb{W}}$, see Lemma 11.3 .

\subsection{Asymptotic behavior at infinity}

To go further into the analysis of Problem (31), we need to study functions $U(\nu, s)$ admitting a behavior at infinity of the form "polynomial + evanescent at infinity". This is our motivation for introducing the following notation.

Definition 4.2 (Property $\mathcal{P}_{\infty}$ ).

A function $U \in \mathbb{V}_{+}^{1}(B)$ will be said to satisfy property $\left(\boldsymbol{P}_{\infty}\right)$ if and only if there exists a function $\mathfrak{U} \in$ $\mathbb{V}_{-}^{1}(B)$, and two functions $p_{ \pm}(\nu, s)$ that admit polynomial dependency with respect to $\nu$, such that

$$
U(\nu, s)=p_{ \pm}(\nu, s)+\mathfrak{U}(\nu, s) \quad \text { for } \pm \nu>2 \pi \text { and } s \in \mathbb{S}^{1} .
$$

Besides, when U satisfies such a property, we set

$$
\ell_{\mathrm{D}}^{ \pm}(U)=p_{ \pm}(0, s) \quad \text { and } \quad \ell_{\mathrm{N}}^{ \pm}(U)=\partial_{\nu} p_{ \pm}(0, s)
$$

as well as

$$
\begin{array}{ll}
\left\langle\ell_{\mathrm{D}}(U)\right\rangle=2 \pi\left(\ell_{\mathrm{D}}^{+}(U)+\ell_{\mathrm{D}}^{-}(U)\right), & {\left[\ell_{\mathrm{D}}(U)\right]=\ell_{\mathrm{D}}^{+}(U)-\ell_{\mathrm{D}}^{-}(U),} \\
\left\langle\ell_{\mathrm{N}}(U)\right\rangle=\frac{1}{2}\left(\ell_{\mathrm{N}}^{+}(U)+\ell_{\mathrm{N}}^{-}(U)\right), & {\left[\ell_{\mathrm{N}}(U)\right]=\ell_{\mathrm{N}}^{+}(U)-\ell_{\mathrm{N}}^{-}(U) .}
\end{array}
$$

Such a property will be commonly encountered during the rest of our study. In particular, solutions to a Laplace equation with a right-hand side that is evanescent at infinity satisfy such a property. The following result is classical from the point of view of Kondratiev's theory, see for example chapter 5 of [21]. However, for the sake of completeness, and since we do not expect our reader to be familiar with such a result, we give a detailed proof.

\section{Proposition 4.3.}

Any $U \in \mathbb{V}_{+}^{1}(B)$ such that $\operatorname{div}\left(\epsilon^{-1} \nabla U\right) \in \mathbb{V}_{+}^{1}(B)^{\prime}$ satisfies Property $\left(\boldsymbol{P}_{\infty}\right)$. Besides, for any such $U$ there exist $a_{ \pm}, b_{ \pm} \in \mathbb{C}$ and $\mathfrak{U} \in \mathbb{V}_{-}^{1}(B)$ such that

$$
U(\nu, s)=a_{ \pm}+b_{ \pm} \nu+\mathfrak{U}(\nu, s) \quad \text { for } \pm \nu>2 \pi, s \in \mathbb{S}^{1} .
$$


Proof. We only prove this result for $\nu>2 \pi$, since the proof for $\nu<-2 \pi$ follows the same lines. Moreover, using a suitable cut-off function if necessary, we assume that $U$ vanishes for $\nu \leq 2 \pi$. Take a $C^{\infty}$ cut-off function $\chi: B \rightarrow[0,1]$ such that $\chi(\nu, s)=\chi(\nu)$, and $\chi(\nu)=0$ for $\nu<\pi$ and $\bar{\chi}(\nu)=1$ for $\nu \geq 2 \pi$. Set $g=-\operatorname{div}\left(\epsilon^{-1} \nabla U\right) \in \mathbb{V}_{+}^{1}(B)^{\prime}$. We apply Laplace transform in $\nu$ and Fourier decomposition in $s$ to the equation $-\operatorname{div}\left(\epsilon^{-1} \nabla U\right)=g$. Set

$$
\begin{array}{lll}
\hat{u}_{k}(\lambda)=\frac{1}{2 \pi} \int_{B} U(\nu, s) e^{-\lambda \nu-i k s} d \nu d s & \text { for } \Re e\{\lambda\}>+1 / 2, & k \in \mathbb{Z}, \\
\hat{g}_{k}(\lambda)=\frac{1}{2 \pi}\left\langle g, e^{-\lambda \nu-i k s}\right\rangle_{+} & \text {for } \Re e\{\lambda\}>-1 / 2, & k \in \mathbb{Z} .
\end{array}
$$

The functions $\chi(\nu) \exp (-\lambda \nu-i k s)$ and $\exp (-\lambda \nu-i k s)$ coincide on the support of $g$ so, since $\chi(\nu) \exp (-\lambda \nu-$ $i k s) \in \mathbb{V}_{+}^{1}(B)$ for $\Re e\{\lambda\}>-1 / 2$, the function $\hat{g}_{k}(\lambda)$ is well defined. Adapting for example the proof of Theorem 7.23 (a) in [27] that relies on Morera's Theorem, it is easy to show that that $\hat{g}_{k}(\lambda)$ is analytic for $\Re e\{\lambda\}>-1 / 2$. Finally, Parseval Identity and Riesz Theorem prove that there exists $C_{1}>0$ such

$$
\sum_{k=-\infty}^{+\infty} \int_{-\infty}^{+\infty} \frac{\left|\hat{g}_{k}(-1 / 2+i \xi)\right|^{2}}{1+k^{2}+\xi^{2}} d \xi \leqslant C_{1}\|g\|_{\left(\mathbb{V}_{+}^{1}\right)^{\prime}}^{2} \quad<+\infty
$$

Now let us take $\chi(\nu) \exp (-\lambda \nu-i k s)$ as a test function that we plug into the variational equation (32). Once again, since $\chi(\nu) \exp (-\lambda \nu-i k s)$ and $\exp (-\lambda \nu-i k s)$ coincide on the support of $U$ and $g$, this yields

$$
\epsilon_{\infty}^{-1}\left(k^{2}-\lambda^{2}\right) \hat{u}_{k}(\lambda)=\hat{g}_{k}(\lambda) \text { for } \Re e\{\lambda\}>1 / 2 .
$$

This identity shows that $\hat{u}_{k}(\lambda)=\epsilon_{\infty}^{-1}\left(k^{2}-\lambda^{2}\right)^{-1} \hat{g}_{k}(\lambda)$ for $\Re e\{\lambda\}>-1 / 2, \lambda \neq k$. As a consequence the functions $\hat{u}_{k}(\lambda)$ can be extended so that: 1) $\hat{u}_{k}(\lambda)$ is analytical for $\Re e\{\lambda\}>-1 / 2$ if $k \neq 0$, and 2) $\hat{u}_{0}(\lambda)$ is analytic over $\{\lambda \in \mathbb{C} \mid \Re e\{\lambda\}>-1 / 2, \lambda \neq 0\}$ with a pole of order 2 at 0 . Applying inversion formula for the Fourier-Laplace transform, we get

$$
U(\nu, s)=\sum_{k \in \mathbb{Z}} \frac{1}{2 i \pi} \int_{\Re e\{\lambda\}=\frac{1}{2}} \hat{u}_{k}(\lambda) e^{\lambda \nu+i k s} d \lambda .
$$

where the contours are parameterized by $\lambda=\frac{1}{2}+i \xi$ and oriented toward $\xi \rightarrow+\infty$. The series converges in $\mathbb{V}_{+}^{1}(B)$ according to Parseval Theorem. Moreover, as the functions $\hat{u}_{k}(\lambda)$ have been meromorphically extended for $\Re e\{\lambda\}>-1 / 2$, we can shift the integration contour to $\lambda=-\frac{1}{2}+i \xi, \xi \in \mathbb{R}$ (oriented toward $\xi \rightarrow+\infty$ ), applying the residue formula for $k=0$. For a justification of this classical operation, see for example Theorem 5.4.1 in [21]. This yields

$$
\begin{aligned}
& U(\nu, s)=\frac{d \hat{g}_{0}}{d \lambda}(0)+\hat{g}_{0}(0) \nu+\mathfrak{V}(\nu, s), \\
& \text { with } \mathfrak{V}(\nu, s)=\sum_{k \in \mathbb{Z}} \frac{1}{2 i \pi} \int_{\Re e\{\lambda\}=-\frac{1}{2}} \frac{\hat{g}_{k}(\lambda)}{k^{2}-\lambda^{2}} e^{\lambda \nu+i k s} d \lambda .
\end{aligned}
$$

Let us show that the function $\mathfrak{U}(\nu, s)=\chi(\nu) \mathfrak{V}(\nu, s)$ belongs to $\mathbb{V}_{-}^{1}(B)$. To prove this, it is sufficient to show that $\mathfrak{V}(\nu, s) \exp (\nu / 2) \in \mathrm{H}^{1}(B)$. Using the parameterization $\lambda=-\frac{1}{2}+i \xi, \xi \in \mathbb{R}$ for the integral in the definition of $\mathfrak{V}(\nu, s)$, we have

$$
e^{\frac{\nu}{2}} \mathfrak{V}(\nu, s)=\frac{1}{2 \pi} \sum_{k \in \mathbb{Z}} \int_{-\infty}^{+\infty} \frac{\hat{g}_{k}\left(-\frac{1}{2}+i \xi\right)}{k^{2}-\left(-\frac{1}{2}+i \xi\right)^{2}} e^{i \xi \nu+i k s} d \xi
$$

It appears that $e^{\frac{\nu}{2}} \mathfrak{V}(\nu, s)$ is exactly the inverse Fourier transform of the function $(\xi, k) \mapsto\left(k^{2}-\left(-\frac{1}{2}+\right.\right.$ $\left.i \xi)^{2}\right)^{-1} \hat{g}_{k}\left(-\frac{1}{2}+i \xi\right)$. Note that there exists a constant $C_{2}>0$ such that $\left(1+\xi^{2}+k^{2}\right) \leq C_{2}\left|k^{2}-\left(-\frac{1}{2}+i \xi\right)^{2}\right|$, $\forall k \in \mathbb{Z}, \forall \xi \in \mathbb{R}$. As a consequence, applying Parseval Theorem once more and taking into account (38), 
we obtain

$$
\begin{aligned}
&\left\|e^{\frac{\nu}{2}} \mathfrak{V}\right\|_{L^{2}(B)}^{2}+\| \nabla\left(e^{\frac{\nu}{2}} \mathfrak{V}\right) \|_{L^{2}(B)}^{2}=\frac{1}{2 \pi} \sum_{k \in \mathbb{Z}} \int_{-\infty}^{+\infty}\left(1+k^{2}+\xi^{2}\right)\left|\frac{\hat{g}_{k}\left(-\frac{1}{2}+i \xi\right)}{k^{2}-\left(-\frac{1}{2}+i \xi\right)^{2}}\right|^{2} d \xi, \\
& \leq \frac{C_{2}^{2}}{2 \pi} \sum_{k \in \mathbb{Z}} \int_{-\infty}^{+\infty} \frac{\left|\hat{g}_{k}\left(-\frac{1}{2}+i \xi\right)\right|^{2}}{1+k^{2}+\xi^{2}} d \xi \leq \frac{C_{1} C_{2}^{2}}{2 \pi}\|g\|_{\left(\mathbb{V}_{+}^{1}\right)^{\prime}}^{2}<+\infty .
\end{aligned}
$$

Straightforward application of Definition 4.2 shows that when $U$ satisfies the assumptions of Proposition 4.3. then $a_{ \pm}=\ell_{\mathrm{D}}^{ \pm}(U)$ and $b_{ \pm}=\ell_{\mathrm{N}}^{ \pm}(U)$. In the case where $U$ satisfies the assumptions of Proposition 4.3 and in addition $U \in \mathbb{W}(B)$, then $\ell_{\mathrm{N}}^{ \pm}(U)=0$ since $\nu \notin \mathbb{W}(B)$.

Now we introduce a particular non-trivial solution to 31 with $g=0$. The following proposition also proves that (31) is ill posed with a kernel of dimension 2 at least.

\section{Proposition 4.4.}

There exists a unique $\mathcal{N} \in \mathbb{V}_{+}^{1}(B)$ such that $\mathcal{N}(\nu, s)-\nu \in \mathbb{W}(B)$ and that satisfies $\operatorname{div}\left(\epsilon^{-1} \nabla \mathcal{N}\right)=0$ in $\mathbb{V}_{+}^{1}(B)^{\prime}$ and $\left\langle\ell_{\mathrm{D}}(\mathcal{N})\right\rangle=0$

Proof. Note that $\operatorname{div}\left(\epsilon^{-1} \nabla \nu\right)=\partial_{\nu}\left(\epsilon^{-1}-\epsilon_{\infty}^{-1}\right) \in \mathbb{V}_{+}^{1}(B)^{\prime}$ since $\epsilon^{-1}-\epsilon_{\infty}^{-1}=0$ for $|\nu|>\pi$. As a consequence, according to Proposition 4.1, there exists a unique $\mathcal{N}_{*} \in \mathbb{W}(B)$ satisfying (33) and such that $\operatorname{div}\left(\epsilon^{-1} \nabla \mathcal{N}_{*}\right)=-\partial_{\nu}\left(\epsilon^{-1}-\epsilon_{\infty}^{-1}\right)$ in $\mathbb{V}_{+}^{1}(B)^{\prime}$. Then it suffices to set $\mathcal{N}=\nu+\mathcal{N}_{*}-\left\langle\ell_{\mathrm{D}}\left(\mathcal{N}_{*}\right)\right\rangle$. Uniqueness of such a function is a consequence of the uniqueness part of Proposition 4.1

Both 1 and $\mathcal{N}$ verify the assumptions of Proposition 4.3. Thus, successively identifying $a_{ \pm}$and $b_{ \pm}$with $\ell_{\mathrm{D}}^{ \pm}(U)$ and $\ell_{\mathrm{N}}^{ \pm}(U)$ for $\pm \nu>2 \pi$ and both for 1 and $\mathcal{N}$ yields the following simple identities

$$
\begin{array}{lllll}
\left\langle\ell_{\mathrm{N}}(1)\right\rangle=0, & {\left[\ell_{\mathrm{N}}(1)\right]=0,} & \left\langle\ell_{\mathrm{D}}(1)\right\rangle=1 \quad \text { and } & {\left[\ell_{\mathrm{D}}(1)\right]=0,} \\
\left\langle\ell_{\mathrm{N}}(\mathcal{N})\right\rangle=1, & {\left[\ell_{\mathrm{N}}(\mathcal{N})\right]=0,} & \left\langle\ell_{\mathrm{D}}(\mathcal{N})\right\rangle=0 & \text { and } & {\left[\ell_{\mathrm{D}}(\mathcal{N})\right] \neq 0 \text { (a priori) } .}
\end{array}
$$

\subsection{General results of well posedness}

Now we show that it is possible to add conditions formulated in terms of the mean and jump operators (36) to Problem (31) so as to obtain a well posed problem where no compatibility condition on the right hand side is required anymore.

\section{Proposition 4.5.}

Consider $g \in \mathbb{V}_{+}^{1}(B)^{\prime}$ and $\alpha, \beta \in \mathbb{C}$ arbitrary. There exists a unique solution to the problem

$$
\begin{aligned}
& \text { Find } U \in \mathbb{V}_{+}^{1}(B) \quad \text { such that } \\
& -\operatorname{div}\left(\epsilon^{-1} \nabla U\right)=g \quad \text { in } \mathbb{V}_{+}^{1}(B)^{\prime}, \\
& \left\langle\ell_{\mathrm{D}}(U)\right\rangle=\alpha \quad \text { and } \quad\left\langle\ell_{\mathrm{N}}(U)\right\rangle=\beta .
\end{aligned}
$$

Moreover there is continuous dependency of the solution with respect to the data, i.e, there exists $C>0$ independent of $\alpha, \beta, g$ such that

$$
\|U\|_{\mathbb{V}_{+}^{1}} \leqslant C\left(|\alpha|+|\beta|+\|g\|_{\left(\mathbb{V}_{+}^{1}\right)^{\prime}}\right) .
$$

Proof.

Uniqueness Assume that $\alpha=\beta=0$ and $g=0$. Let us show that $\left[\ell_{\mathrm{N}}(U)\right]=0$. Consider $\psi_{\mathrm{R}}(\nu)=\psi(\nu / \mathrm{R})$ where $\psi$ is a $C^{\infty}$ function such that $\psi(\nu, s)=\psi(-\nu, s)=\psi(\nu)$, and $\psi(\nu)=0$ for $|\nu|>2 \pi$, and $\psi(\nu)=1$ for $|\nu|<\pi$. Note that since $\left\langle\ell_{\mathrm{D}}(U)\right\rangle=\left\langle\ell_{\mathrm{N}}(U)\right\rangle=0$, according to Proposition 4.3 , there 
exists a function $\mathfrak{U} \in \mathbb{V}_{-}^{1}(B)$ such that $U(\nu, s)= \pm\left(\ell_{\mathrm{D}}^{+}(U)+\ell_{\mathrm{N}}^{+}(U) \nu\right)+\mathfrak{U}(\nu, s)$ for $\pm \nu>2 \pi$. Set $B_{+}=\{(\nu, s) \in B \mid \nu>0\}$. Applying Lemma11.1 to $\mathfrak{U}$ yields

$$
\begin{aligned}
0 & =\lim _{\mathrm{R} \rightarrow \infty} \int_{B} \epsilon^{-1} \nabla U \cdot \nabla \psi_{\mathrm{R}} d s d \nu=\lim _{\mathrm{R} \rightarrow \infty} \int_{B} \epsilon^{-1} \nabla\left(\ell_{\mathrm{N}}^{+}(U) \nu+\ell_{\mathrm{D}}^{+}(U)+\mathfrak{U}(\nu, s)\right) \cdot \nabla \psi_{\mathrm{R}} d s d \nu, \\
& =2 \ell_{\mathrm{N}}^{+}(U) \int_{B_{+}} \epsilon^{-1} \partial_{\nu} \psi_{\mathrm{R}} d s d \nu+\lim _{\mathrm{R} \rightarrow \infty} \int_{B} \epsilon^{-1} \nabla \mathfrak{U} \cdot \nabla \psi_{\mathrm{R}} d s d \nu=4 \pi \epsilon_{\infty}^{-1} \ell_{\mathrm{N}}^{+}(U) .
\end{aligned}
$$

As a consequence $\ell_{\mathrm{N}}^{+}(U)=0$ and this shows that $U \in \mathbb{W}(B)$. Take $C=\int_{\Gamma_{-}} U d s+\int_{\Gamma_{+}} U d s$. Applying Proposition 4.1 to $U-C$ we see that $U=C$. Since $U$ is a constant, we have $U=\left\langle\ell_{\mathrm{D}}(U)\right\rangle=0$ which concludes the uniqueness part of the proof.

Existence Now consider the function $\chi(\nu, s)=1-\psi(\nu)$ where $\psi$ is the cut-off function introduced in the uniqueness part above. This function $\chi$ satisfies

$$
\int_{B} \operatorname{div}\left\{\epsilon^{-1} \nabla(\chi(\nu)|\nu|)\right\} d \nu d s=2 \pi \epsilon_{\infty}^{-1} \int_{-2 \pi}^{+2 \pi} \partial_{\nu}^{2}\{\chi(\nu)|\nu|\} d \nu=4 \pi \epsilon_{\infty}^{-1} .
$$

Set $g_{\infty}=\langle g, 1\rangle_{+}$and $h=g_{\infty} \cdot\left(4 \pi \epsilon_{\infty}^{-1}\right)^{-1} \operatorname{div}\left\{\epsilon^{-1} \nabla(\chi(\nu)|\nu|)\right\}$. We have $\langle h, 1\rangle_{+}=\langle g, 1\rangle_{+}$. Set $g_{*}=g-h$ that satisfies the compatibility condition $\left\langle g_{*}, 1\right\rangle_{+}=0$. Besides $h \in \mathbb{V}_{+}^{1}(B)^{\prime}$ so $g_{*} \in \mathbb{V}_{+}^{1}(B)^{\prime}$ as well. We can apply the existence part of Proposition 4.1 with $g_{*}$ as right-hand side: there exists a function $U_{*} \in \mathbb{W}(B) \subset \mathbb{V}_{+}^{1}(B)$ satisfying (33) such that $-\operatorname{div}\left(\epsilon^{-1} \nabla U_{*}\right)=g_{*}$ in $\mathbb{V}_{+}^{1}(B)^{\prime}$. Taking into account (41), we finally construct a solution to (42) by setting

$$
U=U_{*}-\left\langle\ell_{\mathrm{D}}\left(U_{*}\right)\right\rangle+g_{\infty} \cdot\left(4 \pi \epsilon_{\infty}^{-1}\right)^{-1} \chi(\nu)|\nu|+\alpha+\beta \mathcal{N} .
$$

Continuity To prove (46) for the solution that we have just constructed, it is sufficient to prove that the first three terms in the right hand side of (44) continuously depend on $\alpha, \beta, g$. Clearly $g_{\infty}$ satisfies such an inequality since $\left|\langle g, 1\rangle_{+}\right| \leqslant\|1\|_{\mathbb{V}_{+}^{1}}\|g\|_{\left(\mathbb{V}_{+}^{1}\right)^{\prime}}$. Besides $U_{*}$ also satisfies an inequality similar to (43). Indeed direct estimation shows that there exists $C>0$ such that

$$
\|V\|_{\mathbb{V}_{+}^{1}} \leq C\|V\|_{\mathbb{W}} \quad \forall V \in \mathbb{W}(B) .
$$

Combining this with (34) then proves that there exists $C>0$ independent of $g$ such that $\left\|U_{*}\right\|_{\mathbb{V}_{+}^{1}} \leq$ $C\|g\|_{\left(\mathbb{V}_{+}^{1}\right)^{\prime}}$. There only remain to provide an estimate for $\left\langle\ell_{\mathrm{D}}\left(U_{*}\right)\right\rangle$. Consider the function $V_{*}(\nu, s)=$ $V_{*}(\nu)$ such that $V_{*}(\nu)=0$ if $|\nu| \leq \pi$ and $V_{*}(\nu)=\pi-|\nu|$ if $|\nu|>\pi$. Set $B_{\mathrm{R}}^{ \pm}=\{(\nu, s) \in B \mid \pi \mathrm{R} \leq$ $\pm \nu \leq 2 \pi \mathrm{R}\}$. Observe that $\operatorname{supp}\left(\partial_{\nu} \psi_{\mathrm{R}}\right) \subset B_{\mathrm{R}}^{+} \cup B_{\mathrm{R}}^{-}$. As a consequence, for $\mathrm{R}>\pi$ we have

$$
\begin{aligned}
0 & =\int_{\Gamma_{+}} U_{*} d s+\int_{\Gamma_{-}} U_{*} d s=\int_{B} \nabla\left(\psi_{\mathrm{R}} U_{*}\right) \cdot \nabla V_{*} d \mathbf{x}, \\
& =\int_{B} \psi_{\mathrm{R}} \nabla U_{*} \cdot \nabla V_{*} d \mathbf{x}-\int_{B_{\mathrm{R}}^{+}} U_{*} \partial_{\nu} \psi_{\mathrm{R}} d \mathbf{x}+\int_{B_{\mathrm{R}}^{-}} U_{*} \partial_{\nu} \psi_{\mathrm{R}} d \mathbf{x},
\end{aligned}
$$

where $\Gamma_{ \pm}$have been defined in (33). We can apply Proposition 4.3 to $U_{*}$ and since $U_{*} \in \mathbb{W}(B)$, there exists $\mathfrak{U}_{*} \in \mathbb{V}_{-}^{1}(B)$ such that $U_{*}(\nu, s)=\ell_{\mathrm{D}}^{ \pm}\left(U_{*}\right)+\mathfrak{U}_{*}(\nu, s)$ for $\pm \nu>2 \pi$. As a consequence, using Lemma 11.1 we see that

$$
\lim _{\mathrm{R} \rightarrow+\infty}\left(-\int_{B_{\mathrm{R}}^{+}} U_{*} \partial_{\nu} \psi_{\mathrm{R}} d \mathbf{x}+\int_{B_{\mathrm{R}}^{-}} U_{*} \partial_{\nu} \psi_{\mathrm{R}} d \mathbf{x}\right)=4 \pi\left\langle\ell_{\mathrm{D}}\left(U_{*}\right)\right\rangle .
$$

Also, the decomposition $U_{*}(\nu, s)=\ell_{\mathrm{D}}^{ \pm}\left(U_{*}\right)+\mathfrak{U}_{*}(\nu, s)$ for $\pm \nu>2 \pi$ shows that $\nabla U_{*} \in \mathbb{V}_{-}^{0}(B)$. As a consequence, since $\mathbb{W}(B)$ is dense in $\mathbb{V}_{+}^{1}(B)$ for \|\|$_{\mathbb{V}_{+}^{1}}$ according to Lemma11.3 in appendix, we have $\int_{B} \epsilon^{-1} \nabla U_{*} \nabla V d \mathbf{x}=\left\langle g_{*}, V\right\rangle_{+}, \forall V \in \mathbb{V}_{+}^{1}(B)$. Hence by the dominated convergence theorem

$$
\lim _{\mathrm{R} \rightarrow+\infty} \int_{B} \psi_{\mathrm{R}} \nabla U_{*} \cdot \nabla V_{*} d \mathbf{x}=\epsilon_{\infty} \int_{B} \epsilon^{-1} \nabla U_{*} \cdot \nabla V_{*} d \mathbf{x}=\epsilon_{\infty}\left\langle g_{*}, V_{*}\right\rangle_{+} \cdot
$$


Finally we see that $\left\langle\ell_{\mathrm{D}}\left(U_{*}\right)\right\rangle=-\epsilon_{\infty}\left\langle g_{*}, V_{*}\right\rangle_{+} / 4 \pi$. Since there exists a constant independent of $g$ such that $\left\|g_{*}\right\|_{\left(\mathbb{V}_{+}^{1}\right)^{\prime}} \leq C\|g\|_{\left(\mathbb{V}_{+}^{1}\right)^{\prime}}$, this clearly proves the continuous dependency of $\left\langle\ell_{\mathrm{D}}\left(U_{*}\right)\right\rangle$ with respect to $g$. In conclusion $U$ depends continuously on $\alpha, \beta, g$ with respect to the norm $\|\cdot\|_{\mathbb{V}_{+}^{1}}$.

Remark 4.6. Using Proposition 4.5 it is easy to check that if $g(\nu, s)=\nu^{k}, k \in \mathbb{N}$ then the following problem is well-posed,

$$
\begin{aligned}
& \text { Find } U \in \mathbb{V}_{+}^{1}(B) \quad \text { such that } \\
& -\operatorname{div}\left(\epsilon^{-1} \nabla U\right)=g \quad \text { in } \mathbb{V}_{-}^{1}(B)^{\prime}, \\
& \left\langle\ell_{\mathrm{D}}(U)\right\rangle=\alpha \quad \text { and } \quad\left\langle\ell_{\mathrm{N}}(U)\right\rangle=\beta .
\end{aligned}
$$

Moreover there is continuous dependency of the solution with respect to the data i.e there exists $C>0$ independent of $\alpha, \beta, g$ such that

$$
\|U\|_{\mathbb{V}_{+}^{1}} \leqslant C\left(|\alpha|+|\beta|+\|g\|_{\left(\mathbb{V}_{+}^{1}\right)^{\prime}}\right) .
$$

Whereas $\left\langle\ell_{\mathrm{D}}(U)\right\rangle$ and $\left\langle\ell_{\mathrm{N}}(U)\right\rangle$ should be considered as data in Problem (42), we can provide explicit formula relating directly $\left[\ell_{\mathrm{D}}(U)\right]$ and $\left[\ell_{\mathrm{N}}(U)\right]$ to $\operatorname{div}\left(\epsilon^{-1} \nabla U\right),\left\langle\ell_{\mathrm{D}}(U)\right\rangle$ and $\left\langle\ell_{\mathrm{N}}(U)\right\rangle$. We postpone the proof of the following proposition in appendix since it involves tedious calculus.

\section{Proposition 4.7.}

Assume that $U \in \mathbb{V}_{+}^{1}(B)$ satisfies $-\operatorname{div}\left(\epsilon^{-1} \nabla U\right)=g$ in $\mathbb{V}_{+}^{1}(B)^{\prime}$ for some $g \in \mathbb{V}_{+}^{1}(B)^{\prime}$. Then we have

$$
\begin{aligned}
& {\left[\ell_{\mathrm{N}}(U)\right]=-\frac{\epsilon_{\infty}}{2 \pi}\langle g, 1\rangle_{+} \quad \text { and }} \\
& {\left[\ell_{\mathrm{D}}(U)\right]=\left\langle\ell_{\mathrm{N}}(U)\right\rangle \cdot\left[\ell_{\mathrm{D}}(\mathcal{N})\right]-\frac{\epsilon_{\infty}}{2 \pi}\langle g, \mathcal{N}\rangle_{+} .}
\end{aligned}
$$

When $U$ is solution to (42), a byproduct of Proposition 4.7 above is the continuous dependency of $\ell_{\mathrm{D}}^{ \pm}(U)$ and $\ell_{\mathrm{N}}^{ \pm}(U)$ with respect to $\alpha, \beta, g$.

A natural question is then: is there continuous dependency of the remainder in (4.3) with respect to $\alpha, \beta, g$ ? The answer is positive and it is easy to see it. Indeed according to (46) and Proposition 4.7 there exists a constant $C>0$ independent of $\alpha, \beta, g$ such that

$$
\|\mathfrak{U}\|_{\mathbb{V}_{+}^{1}} \leq C\left(|\alpha|+|\beta|+\|g\|_{\left(\mathbb{V}_{+}^{1}\right)^{\prime}}\right) .
$$

However this estimate is not optimal. A sharper one would involve $\|\mathfrak{U}\|_{\mathbb{V}_{-}^{1}}$ instead of $\|\mathfrak{U}\|_{\mathbb{V}_{+}^{1}}$. Such an estimate can be established as is stated by the following proposition. The proof is postponed to appendix.

\section{Proposition 4.8.}

For $g \in \mathbb{V}_{+}^{1}(B)^{\prime}$ and $\alpha, \beta \in \mathbb{C}$, let $U \in \mathbb{V}_{+}^{1}(B)$ be the unique solution to Problem (42). Let $\chi: \mathbb{R} \rightarrow[0,1]$ be a $C^{\infty}$ cut-off function that satisfies $\chi(\nu)=0$ for $\nu<\pi$ and $\chi(\nu)=1$ for $\nu>2 \pi$. Finally let $\mathfrak{U} \in \mathbb{V}_{-}^{1}(B)$ be defined by

$$
\begin{aligned}
& \mathfrak{U}(\nu, s)=U(\nu, s)-P(\nu), \\
& \text { where } P(\nu)=\chi(\nu)\left[\ell_{\mathrm{D}}^{+}(U)+\ell_{\mathrm{N}}^{+}(U) \nu\right]+\chi(-\nu)\left[\ell_{\mathrm{D}}^{-}(U)+\ell_{\mathrm{N}}^{-}(U) \nu\right] .
\end{aligned}
$$

Then there is continuous dependency of $\mathfrak{U}$ with respect to $\alpha, \beta, g$ : there exists a constant $C>0$ independent of $\alpha, \beta, g$ such that

$$
\|\mathfrak{U}\|_{\mathbb{V}_{-}^{1}} \leq C\left(|\alpha|+|\beta|+\|g\|_{\left(\mathbb{V}_{+}^{1}\right)^{\prime}}\right) .
$$




\section{Expansion of the near field terms at infinity}

In the previous section we considered functions which only depend on the variables $\nu$ and $s$. We are now coming back to the more complex situation of equations (30), where the near field terms $U_{n}$ also depend on the third variable $\tau$. Let us rewrite this system of equations:

$$
\begin{aligned}
& U_{n} \in C^{\infty}\left(\mathbb{S}^{1}, \mathbb{V}_{+}^{1}(B)\right) \\
& A_{0}\left(\partial_{\nu}, \partial_{s}\right) U_{n}=-\sum_{j=1}^{4} \nu^{j} A_{j}\left(\partial_{\nu}, \partial_{s}, \partial_{\tau}\right) U_{n-j} \quad \text { in } \mathbb{V}_{-}^{1}(B)^{\prime}, \quad \forall \tau \in \mathbb{S}^{1} .
\end{aligned}
$$

These equations take, at fixed $n$, a form very close to (31), except that it is parameterized by the third variable $\tau$. Besides, in (30), no condition is prescribed on $\left\langle\ell_{\mathrm{D}}\left(U_{n}\right)\right\rangle$ and $\left\langle\ell_{\mathrm{N}}\left(U_{n}\right)\right\rangle$ (this precise point will be part of the focus of next section).

Remark 5.1. In equation (49), the variable $\tau$ plays the role of a parameter: more precisely, for any $\tau \in \mathbb{S}^{1}$, $U_{n}(., ., \tau) \in \mathbb{V}_{+}^{1}(B)$. Note that, according to the estimates (43, 46, 48), if the right hand side of equation (49) admits $C^{\infty}$ dependency with respect to $\tau$, then the solution to Equation (49) admits $C^{\infty}$ dependency with respect to $\tau$ as well. As we shall see, in the remainder of this paper, near field terms $U_{n}$ will always have $C^{\infty}$ dependency with respect to $\tau$.

At this point of our work, it is interesting to prove that the structure of (49) implies a particular form for the behavior of each $U_{n}$ as $|\nu| \rightarrow \infty$.

\section{Proposition 5.2.}

Consider a family $U_{0}, U_{1}, \ldots U_{n} \in C^{\infty}\left(\mathbb{S}^{1}, \mathbb{V}_{+}^{1}(B)\right)$ satisfying (30). Then each $U_{p}, p=0, \ldots, n$ satisfies Property $\left(\boldsymbol{P}_{\infty}\right)$ and there exist functions $\mathfrak{U}_{p} \in C^{\infty}\left(\mathbb{S}^{1}, \mathbb{V}_{-}^{1}(B)\right)$ such that for $p=0, \ldots, n$, and for $\pm \nu \geq 2 \pi$ there holds

$$
\begin{aligned}
& U_{p}(\nu, s, \tau)= \\
& \sum_{k=0}^{p+1} \nu^{k} \cdot s_{k}^{0}\left(\partial_{\tau}\right) \cdot \ell_{\mathrm{D}}^{ \pm}\left(U_{p-k}(\cdot, \tau)\right)+\sum_{k=0}^{p+1} \nu^{k} \cdot s_{k}^{1}\left(\partial_{\tau}\right) \cdot \ell_{\mathrm{N}}^{ \pm}\left(U_{p+1-k}(\cdot, \tau)\right)+\mathfrak{U}_{p}(\nu, s, \tau) .
\end{aligned}
$$

First of all, despite appearances, the coefficients $\ell_{\mathrm{N}}^{ \pm}\left(U_{p+1}\right)$ do not come into play in Formula (50) since $s_{0}^{1}=0$ according to (17). Besides, Proposition 5.2 shows that, provided that System (30) is satisfied, the function $U_{n}$ satisfy Property $\left(\mathbf{P}_{\infty}\right)$ (although $\nabla \cdot\left(\epsilon^{-1} \nabla U_{n}\right) \notin \mathbb{V}_{+}^{1}(B)^{\prime}$ in general) and their behavior for $|\nu| \rightarrow \infty$ consists in a polynomial part that does not depend on $s$ and an exponentially decreasing part. In addition the polynomial part only depends on a set of functions $\ell_{\mathrm{D}}^{ \pm}\left(U_{n}(\cdot, \tau)\right)$ and $\ell_{\mathrm{N}}^{ \pm}\left(U_{n}(\cdot, \tau)\right)$ that admit a dependency only with respect to $\tau$.

Proof. We proceed by induction. For the first term $U_{0}$ the decomposition (50) is a direct consequence of Proposition 4.3 and the definition of $s_{0}^{1}, s_{1}^{1}, s_{0}^{0}, s_{1}^{0}$ given by (17). Assume now that (50) holds for $p=$ $0,1, \ldots n-1$ and let us prove it for $p=n$. Consider a cut-off function $\chi \in C^{\infty}(B)$ such that $\chi(\nu, s)=$ $\chi(\nu)=1$ for $\nu>2 \pi$, and $\chi(\nu, s)=\chi(\nu)=0$ for $\nu<\pi$. For each $q=0 \ldots n-1$ set

$$
\begin{aligned}
& p_{k}^{ \pm}(\nu, \tau)=\sum_{q=0}^{k+1} \nu^{q} \cdot s_{q}^{0}\left(\partial_{\tau}\right) \cdot \ell_{\mathrm{D}}^{ \pm}\left(U_{k-q}(\cdot, \tau)\right)+\sum_{q=0}^{k+1} \nu^{q} \cdot s_{q}^{1}\left(\partial_{\tau}\right) \cdot \ell_{\mathrm{N}}^{ \pm}\left(U_{k+1-q}(\cdot, \tau)\right), \\
& P_{k}(\nu, \tau)=\chi(\nu) p_{k}^{+}(\nu, \tau)+\chi(-\nu) p_{k}^{-}(\nu, \tau),
\end{aligned}
$$

so that $U_{k}(\nu, s, \tau)=P_{k}(\nu, \tau)+\mathfrak{U}_{k}(\nu, s, \tau)$ for $k=0, \ldots n-1$ and for $|\nu|>2 \pi$. Besides, in order to make notations shorter, we set $c_{q}^{\mathrm{D}, \pm}(\tau)=\ell_{\mathrm{D}}^{ \pm}\left(U_{q}(\cdot, \tau)\right)$ and $c_{q}^{\mathrm{N}, \pm}(\tau)=\ell_{\mathrm{N}}^{ \pm}\left(U_{q}(\cdot, \tau)\right), q=0 \ldots n-1$. 
Using definitions (14) and (17) we see that, for $\nu>\pi$, we have

$$
\begin{aligned}
& \sum_{k=1}^{4} \nu^{k} A_{k}\left(\partial_{\nu}, \partial_{s}, \partial_{\tau}\right) p_{n-k}^{+}(\nu, \tau)=\sum_{k=1}^{+\infty} \nu^{k} \mathscr{A}_{k}\left(\partial_{\nu}, \partial_{\tau}\right) p_{n-k}^{+}(\nu, \tau) \\
& \quad=\sum_{k=1}^{+\infty} \sum_{q=0}^{+\infty} \nu^{k+q} \mathscr{A}_{k}\left(q, \partial_{\tau}\right) \cdot\left(s_{q}^{0}\left(\partial_{\tau}\right) c_{n-k-q}^{\mathrm{D},+}(\tau)+s_{q}^{1}\left(\partial_{\tau}\right) c_{n+1-k-q}^{\mathrm{N},+}(\tau)\right)
\end{aligned}
$$

In the equation above we used the conventions that $c_{q}(\tau)=0$ for $q<0$, and $\mathscr{A}_{k}\left(q, \partial_{\tau}\right)=0$ for $k>4$ and for any $q$, so that the sum contains only finitely many terms. Let us apply the change of index $j=k+q$ and keep the index $k$, which yields

$$
\begin{aligned}
& \sum_{k=1}^{4} \nu^{k} A_{k}\left(\partial_{\nu}, \partial_{s}, \partial_{\tau}\right) p_{n-k}^{+}(\nu, \tau) \\
&= \sum_{j=1}^{+\infty} \nu^{j} \sum_{k=1}^{+\infty} \mathscr{A}_{k}\left(j-k, \partial_{\tau}\right) \cdot\left(s_{j-k}^{0}\left(\partial_{\tau}\right) c_{n-j}^{\mathrm{D},+}(\tau)+s_{j-k}^{1}\left(\partial_{\tau}\right) c_{n+1-j}^{\mathrm{N},+}(\tau)\right), \\
&=-\sum_{j=1}^{+\infty} \nu^{j} \mathscr{A}_{0}(j) \cdot\left(s_{j}^{0}\left(\partial_{\tau}\right) c_{n-j}^{\mathrm{D},+}(\tau)+s_{j}^{1}\left(\partial_{\tau}\right) c_{n+1-j}^{\mathrm{N},+}(\tau)\right)=-A_{0}\left(\partial_{\nu}, \partial_{s}\right) \widetilde{p}_{n}^{+}, \\
& \text {with } \quad \tilde{p}_{n}^{+}(\nu, \tau)=\sum_{q=2}^{n+1} \nu^{q} \cdot\left(s_{q}^{0}\left(\partial_{\tau}\right) c_{n-q}^{\mathrm{D},+}(\tau)+s_{q}^{1}\left(\partial_{\tau}\right) c_{n+1-q}^{\mathrm{N},+}(\tau)\right) .
\end{aligned}
$$

In the last line of this calculus we used the fact that $\mathscr{A}_{0}(0)=\mathscr{A}_{0}(1)=0$. The same calculus holds replacing + by - and introducing a functions $\widetilde{p}_{n}^{-}(\nu, \tau)$. Define $\widetilde{P}_{n}(\nu, s, \tau)=\chi(\nu) \widetilde{p}_{n}^{+}(\nu, \tau)+\chi(-\nu) \widetilde{p}_{n}^{-}(\nu, \tau)$. Since this function is polynomial for $|\nu|$ large enough, explicit calculus shows that $\widetilde{P}_{n}$ satisfies $\left(\mathbf{P}_{\infty}\right)$ and that $\ell_{\mathrm{D}}^{ \pm}\left(\widetilde{P}_{n}(\cdot, \tau)\right)=\ell_{\mathrm{N}}^{ \pm}\left(\widetilde{P}_{n}(\cdot, \tau)\right)=0$ as $\widetilde{p}_{n}^{ \pm}$does not contain any monomial of order 0 or 1 . According to (51)-(52) the function $\widetilde{P}_{n}$ satisfies

$$
Q_{n}:=A_{0}\left(\partial_{\nu}, \partial_{s}\right) \widetilde{P}_{n}+\sum_{k=1}^{4} \nu^{k} A_{k}\left(\partial_{\nu}, \partial_{s}, \partial_{\tau}\right) P_{n-k} \in C^{\infty}\left(\mathbb{S}^{1}, \mathbb{V}_{+}^{1}(B)^{\prime}\right)
$$

Use this remark, and take into account that $\mathfrak{U}_{k} \in C^{\infty}\left(\mathbb{S}^{1}, \mathbb{V}_{+}^{1}(B)^{\prime}\right)$ for $k=0, \ldots, n-1$ to see that $\widetilde{U}_{n}(\nu, s, \tau)=U_{n}(\nu, s, \tau)-\widetilde{P}_{n}(\nu, \tau)$ solves, for each $\tau \in \mathbb{S}^{1}$, a Laplace problem with a right hand side that belongs to $C^{\infty}\left(\mathbb{S}^{1}, \mathbb{V}_{+}^{1}(B)^{\prime}\right)$, namely

$$
A_{0}\left(\partial_{\nu}, \partial_{s}\right) \widetilde{U}_{n}=Q_{n}+\sum_{k=1}^{4} \nu^{k} A_{k}\left(\partial_{\nu}, \partial_{s}, \partial_{\tau}\right) \mathfrak{U}_{n-k} \quad \text { in } \mathbb{R}^{2}
$$

Clearly, for each $\tau \in \mathbb{S}^{1}$, we can apply Proposition 4.3 to $\widetilde{U}_{n}$ which yields the existence of $\widetilde{\mathfrak{U}}_{n} \in$ $C^{\infty}\left(\mathbb{S}^{1}, \mathbb{V}_{-}^{1}(B)\right)$ and $c_{n}^{\mathrm{D}, \pm}, c_{n}^{\mathrm{N}, \pm} \in C^{\infty}\left(\mathbb{S}^{1}\right)$ such that

$$
\widetilde{U}_{n}(\nu, s, \tau)=c_{n}^{\mathrm{D}, \pm}(\tau)+c_{n}^{\mathrm{N}, \pm}(\tau) \nu+\widetilde{\mathfrak{U}}_{n}(\nu, s, \tau) \quad \text { for } \pm \nu>\pi .
$$

Each term of this decomposition has $C^{\infty}$ dependency with respect to $\tau$ according to (46)-(48) and Proposition 4.7. There only remains to recall that $U_{n}=\widetilde{U}_{n}+\widetilde{P}_{n}$, by construction. Moreover, according to the definition (17), we have $s_{1}^{0}=0$ and $s_{0}^{1}=0$. As a consequence, setting $P_{n}(\nu, s, \tau)=$ $\chi(\nu) p_{n}^{+}(\nu, \tau)+\chi(-\nu) p_{n}^{-}(\nu, \tau)$ with

$$
p_{n}^{ \pm}(\nu, \tau)=\widetilde{p}_{n}^{ \pm}(\nu, \tau)+c_{n}^{\mathrm{D}, \pm}(\tau)+c_{n}^{\mathrm{N}, \pm}(\tau) \nu=\sum_{q=0}^{n+1} \nu^{q} \cdot\left(s_{q}^{0}\left(\partial_{\tau}\right) c_{n-q}^{\mathrm{D},+}(\tau)+s_{q}^{1}\left(\partial_{\tau}\right) c_{n+1-q}^{\mathrm{N},+}(\tau)\right)
$$


we have $U_{n}-P_{n} \in C^{\infty}\left(\mathbb{S}^{1}, \mathbb{V}_{-}^{1}\left(\mathbb{R}^{2}\right)\right)$. Finally since $\widetilde{p}_{n}^{ \pm}$has no monomial of order less than 2 , we have $\ell_{\mathrm{D}}^{ \pm}\left(U_{n}(\cdot, \tau)\right)=\ell_{\mathrm{D}}^{ \pm}\left(P_{n}(\cdot, \tau)\right)=c_{n}^{\mathrm{D}, \pm}(\tau)$ and similarly $\ell_{\mathrm{N}}^{ \pm}\left(U_{n}(\cdot, \tau)\right)=\ell_{\mathrm{N}}^{ \pm}\left(P_{n}(\cdot, \tau)\right)=c_{n}^{\mathrm{N}, \pm}(\tau)$. As a consequence, Formula (50) holds for $p=n$.

\section{Matching conditions (Step III)}

In Section 2 we derived formally equations (9) that should hold in order for the functions $u_{n}$ to be the terms of the expansion of $u_{\delta}$ with respect to $\delta$. However, the set of equations (9) is not a well posed problem so it cannot, for the moment, characterize the $u_{n}$ 's. Besides, Adding equations to (9) may lead to a well posed problem, which would yield a definition of the $u_{n}$ as solution of this modified set of equations. A similar remark can be formulated for the $U_{n}$ and the set of equations (30).

The method of matched asymptotics provides such additional equations by means of the matching principle. This principle will yield exactly the proper amount of additional equations needed. Consequently it will lead to a definition of $u_{n}$, and thus to a construction of the expansion of $u_{\delta}$. In spite of several valuable attempts, for example in [33], [19] and [20], there does not exist any generic theory of matched asymptotics at present. As a consequence, this principle can only be justified a posteriori by error estimates that will validate our construction of the expansion $u_{\delta}$.

Recall that we have set $\rho=r-r_{*}$ and $\nu=\rho / \delta$. In the present situation, the matching principle consists in imposing that the expansion of the near field terms for $\nu \rightarrow+\infty$ (resp. $\nu \rightarrow-\infty$ ) should coincide with the expansion of the far field terms for $\rho \rightarrow 0_{+}$(resp. $\rho \rightarrow 0_{-}$). Let us write it formally for $\nu \rightarrow+\infty$ and $\rho \rightarrow 0_{+}$

$$
\begin{aligned}
\sum_{n=0}^{+\infty} \delta^{n} U_{n}(\nu, s, \tau) & \simeq \sum_{n=0}^{+\infty} \delta^{n} \sum_{k=0}^{n+1} \nu^{k}\left(s_{k}^{0}\left(\partial_{\tau}\right) \ell_{\mathrm{D}}^{+}\left(U_{n-k}\right)+s_{k}^{1}\left(\partial_{\tau}\right) \ell_{\mathrm{N}}^{+}\left(U_{n+1-k}\right)\right) \\
& \simeq \sum_{n=0}^{+\infty} \delta^{n} \sum_{k=0}^{n+1}\left(\frac{\rho}{\delta}\right)^{k}\left(s_{k}^{0}\left(\partial_{\tau}\right) \ell_{\mathrm{D}}^{+}\left(U_{n-k}\right)+s_{k}^{1}\left(\partial_{\tau}\right), \ell_{\mathrm{N}}^{+}\left(U_{n+1-k}\right)\right) \\
& \simeq \sum_{n=-1}^{+\infty} \sum_{k=0}^{+\infty} \delta^{n} \rho^{k}\left(s_{k}^{0}\left(\partial_{\tau}\right) \ell_{\mathrm{D}}^{+}\left(U_{n}\right)+s_{k}^{1}\left(\partial_{\tau}\right) \ell_{\mathrm{N}}^{+}\left(U_{n+1}\right)\right) . \\
\sum_{n=0}^{+\infty} \delta^{n} u_{n}(\rho, \theta) & \simeq \sum_{n=-1}^{+\infty} \sum_{k=0}^{+\infty} \delta^{n} \rho^{k}\left(\left.s_{k}^{0}\left(\partial_{\theta}\right) u_{n}\right|_{\Gamma} ^{+}+\left.s_{k}^{1}\left(\partial_{\theta}\right) \partial_{r} u_{n}\right|_{\Gamma} ^{+}\right) .
\end{aligned}
$$

Here we took the convention that $u_{-1}=0$. Comparing both expansions in (53), we see that they coincide provided that $\ell_{\mathrm{D}}^{+}\left(U_{n}(\cdot, \tau=\theta)\right)=\left.u_{n}\right|_{\Gamma} ^{+}$and $\ell_{\mathrm{N}}^{+}\left(U_{n}(\cdot, \tau=\theta)\right)=\left.\partial_{r} u_{n-1}\right|_{\Gamma} ^{+} \forall n \geqslant 0$. Similar conditions can be derived in the same manner for $\nu \rightarrow-\infty$ and $\rho \rightarrow 0_{-}$, so that finally matching conditions take the form

$$
\ell_{\mathrm{D}}^{ \pm}\left(U_{n}(\cdot, \tau=\theta)\right)=\left.u_{n}\right|_{\Gamma} ^{ \pm}(\theta) \quad \text { and } \quad \ell_{\mathrm{N}}^{ \pm}\left(U_{n}(\cdot, \tau=\theta)\right)=\left.\partial_{r} u_{n-1}\right|_{\Gamma} ^{ \pm}(\theta) \quad \forall n \geqslant 0 .
$$

Indeed we have to take into account the equation $\tau=\theta$ when writing the matching equations above. According to the definitions (36), conditions (54) can be rewritten in terms of jump and mean value operators

$$
\forall n \geqslant 0, \quad\left\{\begin{array}{l}
{\left[u_{n}\right]_{\Gamma}=\left[\ell_{\mathrm{D}}\left(U_{n}\right)\right],\left[\partial_{r} u_{n-1}\right]_{\Gamma}=\left[\ell_{\mathrm{N}}\left(U_{n}\right)\right],} \\
\left\langle\ell_{\mathrm{D}}\left(U_{n}\right)\right\rangle=\left\langle u_{n}\right\rangle_{\Gamma},\left\langle\ell_{\mathrm{N}}\left(U_{n}\right)\right\rangle=\left\langle\partial_{r} u_{n-1}\right\rangle_{\Gamma} .
\end{array}\right.
$$

Implicit in the writing of identities (55) is the equation $\tau=\theta$. Recall also that we take convention $u_{-1}=0$. This is the final form of matching conditions that we will keep for the remaining of this analysis. Note that Equations (55) take the form of transmission conditions. 


\section{Recurrent system of equations (Step IV) : existence and unique- ness of the terms of the asymptotic expansion}

Let us summarize the equations that we derived formally, gathering (9), (30) and (55). This yields, for each $n \geq 0$, a system of equations coupling $u_{n}^{ \pm}(r, \theta), U_{n}(\nu, s, \tau=\theta)$ that writes at each step as follows:

$$
\begin{aligned}
& \text { Find } u_{n} \in \mathrm{H}^{1}\left(\Omega_{+}\right) \times \mathrm{H}^{1}\left(\Omega_{-}\right), \quad \text { and } U_{n} \in C_{\#}^{\infty}\left(\mathbb{S}^{1}, \mathbb{V}_{+}^{1}(B)\right) \text { satisfying } \\
& \left\{\begin{array}{l}
\epsilon_{\infty}^{-1} \triangle u_{n}+\omega^{2} \mu_{\infty} u_{n}=f \delta_{n}^{0} \text { in } \Omega_{ \pm} \\
\partial_{r} u_{n}+T u_{n}=0 \quad \text { on } \partial \Omega, \\
{\left[u_{n}\right]_{\Gamma}=\left[\ell_{\mathrm{D}}\left(U_{n}\right)\right] \quad \text { and } \quad\left[\partial_{r} u_{n-1}\right]_{\Gamma}=\left[\ell_{\mathrm{N}}\left(U_{n}\right)\right]}
\end{array}\right. \\
& \left\{\begin{array}{l}
A_{0}\left(\partial_{\nu}, \partial_{s}\right) U_{n}=-\sum_{j=1}^{4} \nu^{j} A_{j} U_{n-j} \quad \text { in } \mathbb{V}_{-}^{1}(B)^{\prime}, \quad \forall \tau \in(0,2 \pi), \\
\left\langle\ell_{\mathrm{D}}\left(U_{n}\right)\right\rangle=\left\langle u_{n}\right\rangle_{\Gamma} \quad \text { and }\left\langle\ell_{\mathrm{N}}\left(U_{n}\right)\right\rangle=\left\langle\partial_{r} u_{n-1}\right\rangle_{\Gamma} .
\end{array}\right.
\end{aligned}
$$

Note that, from now on, we will implicitly assume that $\tau=\theta$. To fully understand the system of equations above recall that $\mathbb{V}_{+}^{1}(B)$ has been defined by (26) and the operators $A_{j}$ have been defined by (24)-(25). The main goal of this section is to prove the following result of well-posedness:

Theorem 7.1. There exist a unique sequence of far field terms $\left(u_{n}\right)_{n \in \mathbb{N}}, u_{n} \in \mathrm{H}^{1}\left(\Omega_{+}\right) \times \mathrm{H}^{1}\left(\Omega_{-}\right)$and a unique sequence of near field terms $\left(U_{n}\right)_{n \in \mathbb{N}}, U_{n} \in C_{\#}^{\infty}\left(\mathbb{S}^{1}, \mathbb{V}_{+}^{1}(B)\right)$, such that recurrent system (56) is satisfied.

Unfortunately it does not seem possible to prove directly Theorem 7.1 indeed, Formulation (56) is not appropriate for a direct recursion proof. More precisely, a careful inspection shows that Problem (56) is not well posed to prove that Problem (56) is well-posed (and completely defines $u_{n+1}$ and $U_{n+1}$ ) under the assumption that $U_{k}$ and $u_{k}$ exist for any $k \leq n$. Roughly speaking, the difficulty arises from the fact that $\left[\ell_{\mathrm{N}}\left(U_{n+2}\right)\right]$ has to be known to define $\left[\partial_{r} u_{n+1}\right]$. This issue seems to be a characteristic of thin transmission layer problems as this was already pointed out at the beginning of Section 3 in [30].

\subsection{Reformulation of recurrent problems}

Well posedness turns out to be much easier to prove with another formulation of Problem (56). Set $U_{n}^{\mathrm{D}}=$ $\left\langle\ell_{\mathrm{D}}\left(U_{n}\right)\right\rangle$ and $U_{n}^{\mathrm{N}}=U_{n}-\left\langle\ell_{\mathrm{D}}\left(U_{n}\right)\right\rangle$ so that we have the following decomposition

$$
U_{n}=U_{n}^{\mathrm{D}}+U_{n}^{\mathrm{N}} \quad \text { with } \quad\left\langle\ell_{\mathrm{D}}\left(U_{n}^{\mathrm{N}}\right)\right\rangle=0 \quad \text { and } \quad U_{n}^{\mathrm{D}}=U_{n}^{\mathrm{D}}(\tau=\theta) .
$$

Note that $\left[\ell_{\mathrm{D}}\left(U_{n}^{\mathrm{D}}\right)\right]=\left[\ell_{\mathrm{N}}\left(U_{n}^{\mathrm{D}}\right)\right]=0$ so that $\left[\ell_{\mathrm{D}}\left(U_{n}\right)\right]=\left[\ell_{\mathrm{D}}\left(U_{n}^{\mathrm{N}}\right)\right]$ and $\left[\ell_{\mathrm{N}}\left(U_{n}\right)\right]=\left[\ell_{\mathrm{N}}\left(U_{n}^{\mathrm{N}}\right)\right]$. Indeed $U_{n}^{\mathrm{D}}$ is assumed to be a function of $\theta$ alone such that $U_{n}^{\mathrm{D}} \in C_{\#}^{\infty}\left(\mathbb{S}^{1}\right)$. We also consider a decomposition of the far field terms in two parts: $u_{n}=u_{n}^{\mathrm{D}}+u_{n}^{\mathrm{N}}$ where

$$
\left\{\begin{array} { l } 
{ \epsilon _ { \infty } ^ { - 1 } \triangle u _ { n } ^ { \mathrm { D } } + \omega ^ { 2 } \mu _ { \infty } u _ { n } ^ { \mathrm { D } } = f \cdot \delta _ { n } ^ { 0 } \text { in } \Omega \backslash \Gamma , } \\
{ \partial _ { r } u _ { n } ^ { \mathrm { D } } + T u _ { n } ^ { \mathrm { D } } = 0 \quad \text { on } \partial \Omega , } \\
{ [ u _ { n } ^ { \mathrm { D } } ] _ { \Gamma } = [ u _ { n } ] _ { \Gamma } , [ \partial _ { r } u _ { n } ^ { \mathrm { D } } ] _ { \Gamma } = 0 . }
\end{array} \quad \left\{\begin{array}{l}
\epsilon_{\infty}^{-1} \triangle u_{n}^{\mathrm{N}}+\omega^{2} \mu_{\infty} u_{n}^{\mathrm{N}}=0 \text { in } \Omega \backslash \Gamma, \\
\partial_{r} u_{n}^{\mathrm{N}}+T u_{n}^{\mathrm{N}}=0 \quad \text { on } \partial \Omega, \\
{\left[u_{n}^{\mathrm{N}}\right]_{\Gamma}=0,\left[\partial_{r} u_{n}^{\mathrm{N}}\right]_{\Gamma}=\left[\partial_{r} u_{n}\right]_{\Gamma} .}
\end{array}\right.\right.
$$

Still we propose to construct $\left(u_{n}, U_{n}\right), n \geq 0$ in a recursive manner, but we do not use Problem (56) solving it at each step $n$ assuming that $\left(u_{0}, U_{0}\right), \ldots,\left(u_{n-1}, U_{n-1}\right)$ are known in order to define $\left(u_{n}, U_{n}\right)$. Instead we consider the change of unknown given by (57)-(58) and rewrite (56) as follows 
Find $u_{n}^{\mathrm{D}} \in \mathrm{H}^{1}\left(\Omega_{+}\right) \times \mathrm{H}^{1}\left(\Omega_{-}\right), \quad u_{n-1}^{\mathrm{N}} \in \mathrm{H}^{1}\left(\Omega_{+}\right) \times \mathrm{H}^{1}\left(\Omega_{-}\right)$

and $U_{n-1}^{\mathrm{D}} \in C_{\#}^{\infty}\left(\mathbb{S}^{1}\right), \quad U_{n}^{\mathrm{N}} \in C_{\#}^{\infty}\left(\mathbb{S}^{1}, \mathbb{V}_{+}^{1}(B)\right)$ satisfying

$$
\begin{aligned}
& \left\{\begin{array}{l}
\epsilon_{\infty}^{-1} \triangle u_{n}^{\mathrm{D}}+\omega^{2} \mu_{\infty} u_{n}^{\mathrm{D}}=f \delta_{n}^{0} \text { in } \Omega_{ \pm}, \\
\partial_{r} u_{n}^{\mathrm{D}}+T u_{n}^{\mathrm{D}}=0 \text { on } \partial \Omega, \\
{\left[u_{n}^{\mathrm{D}}\right]_{\Gamma}-\left[\ell_{\mathrm{D}}\left(U_{n}^{\mathrm{N}}\right)\right]=0 \text { and }\left[\partial_{r} u_{n}^{\mathrm{D}}\right]_{\Gamma}=0,}
\end{array}\right. \\
& \left\{\begin{array}{l}
\epsilon_{\infty}^{-1} \triangle u_{n-1}^{\mathrm{N}}+\omega^{2} \mu_{\infty} u_{n-1}^{\mathrm{N}}=0 \text { in } \Omega_{ \pm}, \\
\partial_{r} u_{n-1}^{\mathrm{N}}+T u_{n-1}^{\mathrm{N}}=0 \text { on } \partial \Omega \\
{\left[u_{n-1}^{\mathrm{N}}\right]_{\Gamma}=0 \text { and }\left[\partial_{r} u_{n-1}^{\mathrm{N}}\right]_{\Gamma}-\left[\ell_{\mathrm{N}}\left(U_{n}^{\mathrm{N}}\right)\right]=0,}
\end{array}\right. \\
& \left\{\begin{aligned}
A_{0}\left(\partial_{\nu}, \partial_{s}\right) U_{n}^{\mathrm{N}} & +\nu A_{1} \cdot U_{n-1}^{\mathrm{D}} \\
& =-\nu A_{1} U_{n-1}^{\mathrm{N}}-\sum_{j=2}^{4} \nu^{j} A_{j} U_{n-j} \quad \text { in } \mathbb{V}_{-}^{1}(B)^{\prime}, \quad \forall \tau \in[0,2 \pi], \\
\left\langle\ell_{\mathrm{D}}\left(U_{n}^{\mathrm{N}}\right)\right\rangle=0 & \text { and }\left\langle\ell_{\mathrm{N}}\left(U_{n}^{\mathrm{N}}\right)\right\rangle-\left\langle\partial_{r} u_{n-1}^{\mathrm{N}}\right\rangle_{\Gamma}=\left\langle\partial_{r} u_{n-1}^{\mathrm{D}}\right\rangle_{\Gamma}
\end{aligned}\right. \\
& \left\{U_{n-1}^{\mathrm{D}}-\left\langle u_{n-1}^{\mathrm{N}}\right\rangle_{\Gamma}=\left\langle u_{n-1}^{\mathrm{D}}\right\rangle_{\Gamma} .\right.
\end{aligned}
$$

Remark 7.2. The two sequences of problems (56) and (59) are equivalent: a sequence $\left(u_{n}, U_{n}\right)_{n \geq 0}$ satisfies (56) if and only if it satisfies (56).

\subsection{Well posedness of recurrent problems}

Our effective recursive procedure for defining $u_{n}, U_{n}$ can be described as follows. At step $n$, assuming that $u_{k}^{\mathrm{D}}, u_{k-1}^{\mathrm{N}}, U_{k}^{N}$ and $U_{k-1}^{\mathrm{D}}$ for $k=0 \ldots n-1$ are known data, we solve Problem (59). This procedure of definition can be considered valid if and only if Problem (59) is well posed.

Proposition 7.3. [Uniqueness]

Assume that $u_{k}^{\mathrm{D}}, u_{k-1}^{\mathrm{N}}, U_{k}^{N}$ and $U_{k-1}^{\mathrm{D}}$ for $k=0 \ldots n-1$ are known data. Then Problem (59) admits at most one solution.

Proof. Let $v^{\mathrm{D}}, v^{\mathrm{N}} \in \mathrm{H}^{1}\left(\Omega_{+}\right) \times \mathrm{H}^{1}\left(\Omega_{-}\right), V^{\mathrm{N}} \in C_{\#}^{\infty}\left(\mathbb{S}^{1}, \mathbb{V}_{+}^{1}(B)\right)$ and $V^{\mathrm{D}} \in C_{\#}^{\infty}\left(\mathbb{S}^{1}\right)$ be a solution to (59) with zero right hand sides. We have to show that $v^{\mathrm{D}}=v^{\mathrm{N}}=0, V^{\mathrm{N}}=0$ and $V^{\mathrm{D}}=0$ necessarily.

First of all, looking at the definition of $A_{1}$ given by (25), we see that $-\nu^{-1} A_{1} \cdot V^{\mathrm{D}}=r_{*}\left(\partial_{\tau} V^{\mathrm{D}}\right) \partial_{s}\left(\epsilon^{-1}\right)$ since $V^{\mathrm{D}}$ is constant with respect to $\nu$ and $s$. Setting $g=r_{*}\left(\partial_{\tau} V^{\mathrm{D}}\right) \partial_{s}\left(\epsilon^{-1}\right)$, we have $\langle g(\cdot, \tau), 1\rangle_{+}=0$. Besides $V^{\mathrm{N}}$ satisfies $\operatorname{div}\left(\epsilon^{-1} \nabla V^{\mathrm{N}}\right)=g$ in $\mathbb{V}_{+}^{1}(B)^{\prime}$ which implies $\left[\ell_{\mathrm{N}}\left(V^{\mathrm{N}}\right)\right]=0$ according to Proposition 4.7. As a consequence $v^{\mathrm{N}}$ is solution to a Helmholtz problem with homogeneous transmission conditions so that $v^{\mathrm{N}}=0$. This directly implies $V^{\mathrm{D}}=0$ hence $g=0$ according to the last equation of (59). Now we see that $V^{\mathrm{N}}$ has to be the solution to a problem that takes the form of (42) with zeros data. This implies $V^{\mathrm{N}}=0$ according to Proposition 4.5 Finally $v^{\mathrm{D}}$ has to satisfy a Helmholtz problem with homogeneous transmission conditions across $\Gamma$. As a consequence $v^{\mathrm{D}}=0$ as well. 


\section{Proposition 7.4 (Existence).}

Assume that $u_{k}^{\mathrm{D}}, u_{k-1}^{\mathrm{N}}, U_{k}^{N}$ and $U_{k-1}^{\mathrm{D}}$ for $k=0 \ldots n-1$ are known data. Then Problem (59) admits at least one solution.

Proof. We focus only on the construction of $u_{n-1}^{\mathrm{N}}, U_{n}^{N}$ and $U_{n-1}^{\mathrm{D}}$ since, once $U_{n}^{N}$ has been defined, the definition of $u_{n}^{\mathrm{D}}$ amounts to solving a classical transmission problem which does not raise any technical difficulty. Observe that the equation ruling $U_{n}^{N}$ fits the framework of Proposition 5.2, and in particular $U_{0}, \ldots, U_{n-1}$ take the form (50). Introduce

$Q_{n}(\nu, \tau)=\chi(\nu) p_{n}^{+}(\nu, \tau)+\chi(-\nu) p_{n}^{-}(\nu, \tau) \quad$ with $p_{n}^{ \pm}=\sum_{k=2}^{n+1} \nu^{k}\left(s_{k}^{0}\left\{\ell_{\mathrm{D}}^{ \pm}\left(U_{n-k}\right)\right\}+s_{k}^{1}\left\{\ell_{\mathrm{N}}^{ \pm}\left(U_{n+1-k}\right)\right\}\right)$.

Since $p_{n}^{+}$do not contain any monomial of degree less than 2 , we have $\ell_{\mathrm{D}}^{ \pm}\left(Q_{n}\right)=0$ and $\ell_{\mathrm{N}}^{ \pm}\left(Q_{n}\right)=0$. Moreover, according to Proposition 5.2 and Equation (51)-(52), the function $Q_{n}(\nu, \tau)$ solves the recurrent equations ruling $U_{n}^{N}$ up to a right hand side that exponentially decreases at infinity. More precisely

$$
g:=\nu^{-2} A_{0}\left(\partial_{\nu}, \partial_{s}\right) Q_{n}+\nu^{-1} A_{1} U_{n-1}^{\mathrm{N}}+\sum_{j=2}^{4} \nu^{j-2} A_{j} U_{n-j} \in C^{\infty}\left(\mathbb{S}^{1}, \mathbb{V}_{+}^{1}(B)^{\prime}\right) .
$$

According to Proposition 4.5, there exists a unique $R_{n} \in C_{\#}^{\infty}\left(\mathbb{S}^{1}, \mathbb{V}_{+}^{1}(B)\right)$ satisfying $\nu^{-2} A_{0}\left(\partial_{\nu}, \partial_{s}\right) R_{n}$ $=-g$ in $\mathbb{V}_{+}^{1}(B)^{\prime}$, and $\left\langle\ell_{\mathrm{D}}\left(R_{n}\right)\right\rangle=0$, and $\left\langle\ell_{\mathrm{N}}\left(R_{n}\right)>=0\right.$. Finally setting $V_{n}=R_{n}+Q_{n}$, since $\ell_{\mathrm{D}}^{ \pm}\left(V_{n}\right)=\ell_{\mathrm{D}}^{ \pm}\left(R_{n}\right)$ and $\ell_{\mathrm{N}}^{ \pm}\left(V_{n}\right)=\ell_{\mathrm{N}}^{ \pm}\left(R_{n}\right)$ by construction, we have

$$
A_{0}\left(\partial_{\nu}, \partial_{s}\right) V_{n}=-\nu A_{1} U_{n-1}^{\mathrm{N}}-\sum_{j=2}^{4} \nu^{j} A_{j} U_{n-j} \text { in } \mathbb{R}^{2} \quad \text { and } \quad\left\langle\ell_{\mathrm{D}}\left(V_{n}\right)\right\rangle=0, \quad\left\langle\ell_{\mathrm{N}}\left(V_{n}\right)\right\rangle=0 .
$$

Note however that, a priori, we have $\left[\ell_{\mathrm{D}}\left(V_{n}\right)\right] \neq 0$ and $\left[\ell_{\mathrm{N}}\left(V_{n}\right)\right] \neq 0$. Now we define $u_{n-1}^{\mathrm{N}} \in \mathrm{H}^{1}\left(\Omega_{+}\right) \times$ $\mathrm{H}^{1}\left(\Omega_{-}\right)$as the unique function satisfying the following system of equations

$$
\left\{\begin{array}{l}
\epsilon_{\infty}^{-1} \triangle u_{n-1}^{\mathrm{N}}+\omega^{2} \mu_{\infty} u_{n-1}^{\mathrm{N}}=0 \text { in } \Omega_{ \pm}, \\
\partial_{r} u_{n-1}^{\mathrm{N}}+T u_{n-1}^{\mathrm{N}}=0 \quad \text { on } \partial \Omega \\
{\left[u_{n-1}^{\mathrm{N}}\right]_{\Gamma}=0 \text { and }\left[\partial_{r} u_{n-1}^{\mathrm{N}}\right]_{\Gamma}=\left[\ell_{\mathrm{N}}\left(V_{n}\right)\right] .}
\end{array}\right.
$$

Of course, we will have to verify a posteriori that such a $u_{n-1}^{\mathrm{N}}$ satisfies the first system of equations of (59). Now that $u_{n-1}^{\mathrm{N}}$ is constructed, we directly set $U_{n-1}^{\mathrm{D}}=\left\langle u_{n-1}^{\mathrm{N}}\right\rangle_{\Gamma}+\left\langle u_{n-1}^{\mathrm{D}}\right\rangle_{\Gamma}$. There only remains to construct $U_{n}^{\mathrm{N}}$. For this purpose, we introduce a last intermediate function, denoted $W_{n}$, that is defined as the unique function satisfying

$$
\begin{aligned}
& W_{n} \in C^{\infty}\left(\mathbb{S}^{1}, \mathbb{V}_{+}^{1}(B)\right) \\
& \nu^{-2} A_{0}\left(\partial_{\nu}, \partial_{s}\right) W_{n}=-\nu^{-1} A_{1} U_{n-1}^{\mathrm{D}} \quad \text { in } \mathbb{V}_{-}^{1}(B)^{\prime}, \\
& \left\langle\ell_{\mathrm{D}}\left(W_{n}\right)\right\rangle=0 \text { and }\left\langle\ell_{\mathrm{N}}\left(W_{n}\right)\right\rangle=\left\langle\partial_{r} u_{n-1}^{\mathrm{N}}\right\rangle_{\Gamma}+\left\langle\partial_{r} u_{n-1}^{\mathrm{D}}\right\rangle_{\Gamma} .
\end{aligned}
$$

This problem is well posed. Indeed according to the definition of $A_{0}$ and $A_{1}$ given by (24) and (25) we have $\nu^{-2} A_{0}\left(\partial_{\nu}, \partial_{s}\right) W_{n}=\operatorname{div}\left(\epsilon^{-1} \nabla W_{n}\right)$ on the one hand, and $\nu^{-1} A_{1} U_{n-1}^{\mathrm{D}}=r_{*} \partial_{\theta}\left(U_{n-1}^{\mathrm{D}}\right) \partial_{s}\left(\epsilon^{-1}\right)$ (recall that $U_{n-1}^{\mathrm{D}}$ depends only on $\theta$ by definition). Setting $h=\nu^{-1} A_{1} U_{n-1}^{\mathrm{D}}$, this implies in particular that $h \in \mathbb{V}_{+}^{1}(B)^{\prime}$ and that $\left\langle h, 1>_{+}=0\right.$. As a consequence, applying Proposition 4.7 to $W_{n}$ yields $\left[\ell_{\mathrm{N}}\left(W_{n}\right)\right]=0$. We finally set

$$
U_{n}^{\mathrm{N}}=V_{n}+W_{n} .
$$

The function $U_{n}^{\mathrm{N}}$ satisfies the third system of equations in (59). Besides we have $\left[\partial_{r} u_{n-1}^{\mathrm{N}}\right]=\left[\ell_{\mathrm{N}}\left(V_{n}\right)\right]=$ $\left[\ell_{\mathrm{N}}\left(U_{n}\right)\right]$ which confirms that $u_{n-1}^{\mathrm{N}}$ properly satisfies also the equations of [59]. This concludes the construction of a solution, hence the existence. 


\section{Approximate transmission conditions}

In the previous part, we proved that the near and far field terms are well defined. We may provide a refined analysis by trying to uncouple the equations ruling the far field on the one hand, and the near field on the other hand. The main motivation for this will be the derivation of approximate transmission conditions for Problem (1) up to any order.

We would like to reformulate equations (56) as a system involving only far field terms, where transmission conditions would not contain any direct dependency on the near field terms. To do this, we need to make the dependency of the $U_{n}$ 's with respect to the variable $\tau$ as explicit as possible. Actually, a careful examination of equations (56) should convince the reader that such a dependency is inherited from the terms $\left\langle u_{k}\right\rangle_{\Gamma}$ and $\left\langle\partial_{r} u_{k}\right\rangle_{\Gamma}, k \leq n$ (note the linearity dependence of the near field equations $\left.23 \mid 24,25\right)$ in term of partial derivatives in the variable $\tau$ ).

Keeping in mind the previous comments, let us introduce intermediate notation. For $\xi \in \mathbb{C}$, define $\hat{A}_{j}(\xi)$ as the operator $A_{j}$ where $\partial_{\tau}$ has been replaced by $\xi$. In other words, we have $\hat{A}_{0}(\xi)=A_{0}, \hat{A}_{3}(\xi)=A_{3}$ and $\hat{A}_{4}(\xi)=A_{4}$, whereas

$$
\begin{aligned}
& \hat{A}_{1}(\xi) \cdot U(\nu, s)=2 \nu^{2} \partial_{\nu}\left(\epsilon^{-1} \partial_{\nu} U\right)+\epsilon^{-1} \nu \partial_{\nu} U+\xi\left[\nu \partial_{s}\left(\epsilon^{-1} U\right)+\nu\left(\epsilon^{-1} \partial_{s} U\right)\right], \\
& \hat{A}_{1}(\xi) \cdot U(\nu, s)=\nu \partial_{\nu}\left(\epsilon^{-1} \nu \partial_{\nu} U\right)+r_{*}^{2} \mu \omega^{2} U+\xi^{2} \epsilon^{-1} U .
\end{aligned}
$$

Now we construct two families of functions $\left(\mathcal{U}_{n}(\xi)\right)_{n \geq 0}$ and $\left(\mathcal{N}_{n}(\xi)\right)_{n \geq 0}$. Each $\mathcal{U}_{n}(\xi)$ and $\mathcal{N}_{n}(\xi)$ is assumed to be polynomial with respect to $\xi$, with coefficients in $\mathbb{V}_{1}^{+}(B)$ namely

$$
\mathcal{U}_{n}(\nu, s, \xi)=\sum_{k=0}^{k_{n}} \xi^{k} \mathcal{U}_{n}^{k}(\nu, s) \quad \text { and } \quad \mathcal{N}_{n}(\nu, s, \xi)=\sum_{k=0}^{k_{n}} \xi^{k} \mathcal{N}_{n}^{k}(\nu, s) \quad \text { with } \quad \mathcal{U}_{n}^{k}, \mathcal{N}_{n}^{k} \in \mathbb{V}_{1}^{+}(B)
$$

We define both families $\left(\mathcal{U}_{n}(\xi)\right)_{n \geq 0}$ and $\left(\mathcal{N}_{n}(\xi)\right)_{n \geq 0}$ by a recursive procedure formulated by means of the operators $\hat{A}_{j}(\xi), j=0 \ldots 4$ as follows

\section{Definition 8.1.}

$-\mathcal{U}_{0}(\nu, s, \xi)=1$

- For each $n \geq 0, \xi \in \mathbb{C}$, the function $\mathcal{U}_{n}(\xi)$ is the unique element of $\mathbb{V}_{1}^{+}(B)$ that satisfies,

$$
\left\{\begin{array}{l}
A_{0}\left(\partial_{\nu}, \partial_{s}\right) \mathcal{U}_{n}(\nu, s, \xi)=-\sum_{j=1}^{4} \nu^{j} \hat{A}_{j}\left(\partial_{\nu}, \partial_{s}, \xi\right) \mathcal{U}_{n-j}(\nu, s, \xi) \text { in } \mathbb{V}_{1}^{-}(B)^{\prime}, \\
\left\langle\ell_{\mathrm{D}}\left(\mathcal{U}_{n}(\xi)\right)\right\rangle=0 \\
\left\langle\ell_{\mathrm{N}}\left(\mathcal{U}_{n}(\xi)\right)\right\rangle=0 .
\end{array}\right.
$$

\section{Definition 8.2.}

$-\mathcal{N}_{0}(\nu, s, \xi)=\mathcal{N}(\nu, s)$,

- For each $n \geq 0, \xi \in \mathbb{C}$, the function $\mathcal{N}_{n}(\xi)$ is the unique element of $\mathbb{V}_{1}^{+}(B)$ that satisfies,

$$
\left\{\begin{array}{l}
A_{0}\left(\partial_{\nu}, \partial_{s}\right) \mathcal{N}_{n}(\nu, s, \xi)=-\sum_{j=1}^{4} \nu^{j} \hat{A}_{j}\left(\partial_{\nu}, \partial_{s}, \xi\right) \mathcal{N}_{n-j}(\nu, s, \xi) \text { in } \mathbb{V}_{1}^{-}(B)^{\prime} \\
\left\langle\ell_{\mathrm{D}}\left(\mathcal{N}_{n}(\xi)\right)\right\rangle=0 \\
\left\langle\ell_{\mathrm{N}}\left(\mathcal{N}_{n}(\xi)\right)\right\rangle=0 .
\end{array}\right.
$$


As usual, we took as a convention in the above definitions, that $\mathcal{U}_{n}(\xi)=\mathcal{N}_{n}(\xi)=0$ if $n \leq-1$. We may consider that these definitions are valid only if Problems (61) and (62) are well posed. Such a verification is achieved using Proposition 4.5 and Remark 4.6 Besides the linearity of Problems (61) and (62) ensures that the dependency of $\mathcal{U}_{n}(\xi)$ and $\mathcal{N}_{n}(\xi)$ is polynomial. A brief induction shows that the degree of $\mathcal{U}_{n}(\xi)$ and $\mathcal{N}_{n}(\xi)$ as polynomials in $\xi$ cannot be greater than $n$.

It is clear from $[61)$, by a recurrence argument, that $\mathcal{U}_{n}(\xi)$ satisfies Property $\left(\mathbf{P}_{\infty}\right)$ for any $n \geq 0$. Similarly $<\ell_{\mathrm{D}}\left(\mathcal{U}_{n}(\xi)\right)>$ and $<\ell_{\mathrm{N}}\left(\mathcal{U}_{n}(\xi)\right)>$ are polynomials in $\xi$, and the conditions stated in 610 that hold for any $\xi \in \mathbb{C}$, prove that $<\ell_{\mathrm{D}}\left(\mathcal{U}_{n}^{k}\right)>=<\ell_{\mathrm{N}}\left(\mathcal{U}_{n}^{k}\right)>=0$ for any $k \geq 0$ and any $n \geq 1$. Note however that, by construction, we have $\left.<\ell_{\mathrm{D}}\left(\mathcal{U}_{0}\right)\right\rangle=1$ and $<\ell_{\mathrm{N}}\left(\mathcal{U}_{0}\right)>=0$. Similar remarks may be formulated, mutatis mutandis, about $\left(\mathcal{N}_{n}(\xi)\right)_{n \geq 0}$.

The functions $\left(\mathcal{U}_{n}(\xi)\right)_{n \geq 0}$ and $\left(\mathcal{N}_{n}(\xi)\right)_{n \geq 0}$ may be used to construct solutions to equations (30): we first naturally associate with $\overline{\mathcal{U}}_{n}(\xi)$ and $\mathcal{N}_{n}(\xi)$, the differential operators in $\tau$

$$
\mathcal{U}_{n}\left(\nu, s, \partial_{\tau}\right)=\sum_{k=0}^{n} \mathcal{U}_{n}^{k}(\nu, s) \partial_{\tau}^{k}, \quad \mathcal{N}_{n}\left(\nu, s, \partial_{\tau}\right)=\sum_{k=0}^{n} \mathcal{N}_{n}^{k}(\nu, s) \partial_{\tau}^{k}
$$

as well as $\ell_{\mathrm{D}}^{ \pm}\left(\mathcal{U}_{n}\left(\partial_{\tau}\right)\right), \ell_{\mathrm{N}}^{ \pm}\left(\mathcal{U}_{n}\left(\partial_{\tau}\right)\right), \ell_{\mathrm{D}}^{ \pm}\left(\mathcal{N}_{n}\left(\partial_{\tau}\right)\right.$ and $\ell_{\mathrm{N}}\left(\mathcal{D}_{n}\left(\partial_{\tau}\right)\right)$ :

$$
\begin{array}{ll}
\ell_{\mathrm{D}}^{ \pm}\left(\mathcal{U}_{n}\left(\partial_{\tau}\right)\right)=\sum_{k=0}^{n} \ell_{\mathrm{D}}^{ \pm}\left(\mathcal{U}_{n}^{k}\right) \partial_{\tau}^{k}, & \ell_{\mathrm{N}}^{ \pm}\left(\mathcal{U}_{n}\left(\partial_{\tau}\right)\right)=\sum_{k=0}^{n} \ell_{\mathrm{N}}^{ \pm}\left(\mathcal{U}_{n}^{k}\right) \partial_{\tau}^{k}, \\
\left.\ell_{\mathrm{D}}^{ \pm}\left(\mathcal{U}_{n}\left(\partial_{\tau}\right)\right)=\sum_{k=0}^{n} \ell_{\mathrm{D}}^{ \pm}\left(\mathcal{U}_{n}^{k}\right)\right] \partial_{\tau}^{k}, & \ell_{\mathrm{N}}^{n}\left(\mathcal{U}_{n}\left(\partial_{\tau}\right)\right)=\sum_{k=0}^{n} \ell_{\mathrm{N}}\left(\mathcal{U}_{n}^{k}\right), \partial_{\tau}^{k} .
\end{array}
$$

Now, take any function $c(\tau) \in C_{\#}^{\infty}\left(\mathbb{S}^{1}\right)$, and consider the functions

$$
C_{n}(\nu, s, \tau)=\mathcal{U}_{n}\left(\nu, s, \partial_{\tau}\right) c(\tau)=\sum_{k=0}^{n} \mathcal{U}_{n}^{k}(\nu, s) \partial_{\tau}^{k} c(\tau) \quad n \geq 0
$$

where $\mathcal{U}_{n}^{k}(\nu, s)$ are the coefficients of $\mathcal{U}_{n}(\nu, s, \xi)$ as polynomial in $\xi$. It is easy to check that the functions $C_{n}(\nu, s, \tau)$ satisfy recurrence equations of the form (30). Indeed we have

$$
\left\{\begin{array}{l}
\sum_{j=0}^{4} \nu^{j} A_{j}\left(\partial_{\nu}, \partial_{s}, \partial_{\tau}\right) C_{n-j}(\nu, s, \tau)=\left[\sum_{j=0}^{4} \nu^{j} A_{j}\left(\partial_{\nu}, \partial_{s}, \partial_{\tau}\right) \mathcal{U}_{n-j}\left(\nu, s, \partial_{\tau}\right)\right] c(\tau)=0 \\
\left\langle\ell_{\mathrm{N}}\left(C_{n}(\cdot, \tau)\right)\right\rangle=0 \quad \forall n \geq 0, \\
\left\langle\ell_{\mathrm{D}}\left(C_{n}(\cdot, \tau)\right)\right\rangle=0 \quad \forall n \geq 1 \quad \text { and } \quad\left\langle\ell_{\mathrm{D}}\left(C_{0}(\cdot, \tau)\right)\right\rangle=c(\tau) .
\end{array}\right.
$$

Similar remarks can be formulated, replacing $\mathcal{U}_{n}(\xi)$ by $\mathcal{N}_{n}(\xi)$, and setting $C_{n}(\nu, s, \tau)=\mathcal{N}_{n}\left(\nu, s, \partial_{\tau}\right) c(\tau)$. In this case we have $\left\langle\ell_{\mathrm{D}}\left(C_{n}(\cdot, \tau)\right)\right\rangle=0$ for all $n \geq 0,\left\langle\ell_{\mathrm{N}}\left(C_{n}(\cdot, \tau)\right)\right\rangle=0$ for $n \geq 1$, and $\left\langle\ell_{\mathrm{N}}\left(C_{0}(\cdot, \tau)\right)\right\rangle=$ $c(\tau)$. Now we state a result about the terms of the asymptotic expansion that we defined as the solutions to (59).

Theorem 8.3.

Let $\left(u_{n}, U_{n}\right)_{n \geq 0}$ be the sequence that were recursively constructed as the solution to (59). These functions 
also satisfy, on the one hand,

$$
\left\{\begin{array}{l}
\varepsilon_{\infty}^{-1} \Delta u_{n}+\omega^{2} \mu_{\infty} u_{n}=\delta_{n}^{0} f \quad \text { in } \Omega_{+} \cup \Omega_{-}, \\
\partial_{r} u_{n-1}+i \omega u_{n-1}=0 \quad \text { on } \partial \Omega, \\
{\left[u_{n}\right]_{\Gamma}=\sum_{j=1}^{n}\left(\sum_{k=0}^{j}\left[\ell_{\mathrm{D}}\left(\mathcal{U}_{j}^{k}\right)\right] \partial_{\theta}^{k}\right)\left\langle u_{n-j}\right\rangle_{\Gamma}+\sum_{j=0}^{n-1}\left(\sum_{k=0}^{j}\left[\ell_{\mathrm{D}}\left(\mathcal{N}_{j}^{k}\right)\right] \partial_{\theta}^{k}\right)\left\langle\partial_{r} u_{n-1-j}\right\rangle_{\Gamma} \quad(a),} \\
{\left[\partial_{r} u_{n}\right]_{\Gamma}=\sum_{j=2}^{n+1}\left(\sum_{k=0}^{j}\left[\ell_{\mathrm{N}}\left(\mathcal{U}_{j}^{k}\right)\right] \partial_{\theta}^{k}\right)\left\langle u_{n+1-j}\right\rangle_{\Gamma}+\sum_{j=1}^{n}\left(\sum_{k=0}^{j}\left[\ell_{\mathrm{N}}\left(\mathcal{N}_{j}^{k}\right)\right] \partial_{\theta}^{k}\right)\left\langle\partial_{r} u_{n-1-j}\right\rangle_{\Gamma} \quad(b),}
\end{array}\right.
$$

and on the other hand,

$$
U_{n}(\nu, s, \tau)=\sum_{j=0}^{n} \mathcal{U}_{j}\left(\nu, s, \partial_{\tau}\right)\left\langle u_{n-j}\right\rangle_{\Gamma}(\tau)+\sum_{j=0}^{n-1} \mathcal{N}_{j}\left(\nu, s, \partial_{\tau}\right)\left\langle\partial_{r} u_{n-1-j}\right\rangle_{\Gamma}(\tau) .
$$

Proof. First, let us remark that for any $n \in \mathbb{N}$, problem (64) is well posed, since the right hand side only depends on $u_{n-1}, u_{n-2}, \ldots u_{0}$. To prove the proposition, it is thus sufficient to show that the sequence $\left(u_{n}, U_{n}\right)_{n \in \mathbb{N}}$ defined by (64)-(65) is solution to (56). The jump relations in (64) state that we have $\left[u_{n}\right]_{\Gamma}=$ $\left[\ell_{\mathrm{D}}\left(U_{n}\right)\right]=0$, and $\left[\partial_{r} u_{n}\right]_{\Gamma}=\left[\ell_{\mathrm{N}}\left(U_{n+1}\right)\right]$. Recall that $\left\langle\ell_{\mathrm{D}}\left(\mathcal{N}_{j}^{k}\right)\right\rangle=0$ for all $k, n \geq 0$, and that $\left\langle\ell_{\mathrm{D}}\left(\mathcal{U}_{j}^{k}\right)\right\rangle=0$ if $j \neq 0$, and $\left\langle\ell_{\mathrm{D}}\left(\mathcal{U}_{0}^{0}\right)\right\rangle=1$ according to the construction of $\mathcal{U}_{n}(\nu, s, \xi)$ and $\mathcal{N}_{n}(\nu, s, \xi)$. As a consequence we have

$$
\left\langle\ell_{\mathrm{D}}\left(U_{n}(\cdot, \tau)\right)\right\rangle=\sum_{j=0}^{n} \sum_{k=0}^{j}\left\langle\ell_{\mathrm{D}}\left(\mathcal{U}_{j}^{k}\right)\right\rangle \partial_{\tau}^{k}\left\langle u_{n-j}\right\rangle_{\Gamma}+\sum_{j=0}^{n-1} \sum_{k=0}^{j}\left\langle\ell_{\mathrm{D}}\left(\mathcal{N}_{j}^{k}\right)\right\rangle\left\langle\partial_{r} u_{n-1-j}\right\rangle_{\Gamma}=\left\langle u_{n}\right\rangle_{\Gamma}+0 .
$$

Similarly, recall that $\left\langle\ell_{\mathrm{N}}\left(\mathcal{U}_{j}^{k}\right)\right\rangle=0$ for all $k, n \geq 0$, and that $\left\langle\ell_{\mathrm{N}}\left(\mathcal{N}_{j}^{k}\right)\right\rangle=0$ if $j \neq 0$, and $\left\langle\ell_{\mathrm{N}}\left(\mathcal{N}_{0}^{0}\right)\right\rangle=1$. This yields

$$
\left\langle\ell_{\mathrm{N}}\left(U_{n}(\cdot, \tau)\right)\right\rangle=\sum_{j=0}^{n} \sum_{k=0}^{j}\left\langle\ell_{\mathrm{N}}\left(\mathcal{U}_{j}^{k}\right)\right\rangle \partial_{\tau}^{k}\left\langle u_{n-j}\right\rangle_{\Gamma}+\sum_{j=0}^{n-1} \sum_{k=0}^{j}\left\langle\ell_{\mathrm{N}}\left(\mathcal{N}_{j}^{k}\right)\right\rangle\left\langle\partial_{r} u_{n-1-j}\right\rangle_{\Gamma}=0+\left\langle\partial_{r} u_{n-1}\right\rangle_{\Gamma} .
$$

To sum up we have just verified that the matching conditions (55) are satisfied by functions $\left(u_{n}, U_{n}\right)$ that would be defined by (64)-(65). There only remains to verify that the sequence $U_{0}, U_{1}, \ldots$ defined by (65) satisfies (30).

$$
\begin{aligned}
\sum_{j=0}^{4} \nu^{j} A_{j}\left(\partial_{\nu}, \partial_{s}, \partial_{\tau}\right) U_{n-j}(\nu, s, \tau)= & \sum_{j=0}^{4} \nu^{j} A_{j}\left(\partial_{\nu}, \partial_{s}, \partial_{\tau}\right) \sum_{k=0}^{n-j} \mathcal{U}_{k}\left(\nu, s, \partial_{\tau}\right)\left\langle u_{n-j-k}\right\rangle_{\Gamma}(\tau) \\
& +\sum_{j=0}^{4} \nu^{j} A_{j}\left(\partial_{\nu}, \partial_{s}, \partial_{\tau}\right) \sum_{k=0}^{n-1-j} \mathcal{N}_{k}\left(\nu, s, \partial_{\tau}\right)\left\langle u_{n-j-k}\right\rangle_{\Gamma}(\tau) .
\end{aligned}
$$

We show that both sums above are equal to 0 . We prove it only for the first sum, as the second sum may be treated exactly in the same manner. Once again, let us take the convention that $A_{j}=0$ for $j<0$ or $j>4$, and $u_{n}=0$ for $n<0$. We have

$$
\begin{aligned}
\sum_{j=0}^{4} \nu^{j} A_{j}\left(\partial_{\nu}, \partial_{s}, \partial_{\tau}\right) \sum_{k=0}^{n-j} \mathcal{U}_{k} & \left(\nu, s, \partial_{\tau}\right)\left\langle u_{n-j-k}\right\rangle_{\Gamma}(\tau) \\
(\text { with } p=j+k) \quad & =\sum_{j=0}^{4} \nu^{j} A_{j}\left(\partial_{\nu}, \partial_{s}, \partial_{\tau}\right) \sum_{p=0}^{n} \mathcal{U}_{p-j}\left(\nu, s, \partial_{\tau}\right)\left\langle u_{n-p}\right\rangle_{\Gamma}(\tau), \\
& =\sum_{p=0}^{n}\left[\sum_{j=0}^{4} \nu^{j} A_{j}\left(\partial_{\nu}, \partial_{s}, \partial_{\tau}\right) \mathcal{U}_{p-j}\left(\nu, s, \partial_{\tau}\right)\right]\left\langle u_{n-p}\right\rangle_{\Gamma}(\tau)=0 .
\end{aligned}
$$


In the last line of the above calculus, we used (63). The same calculus can be achieved with the second sum in the right hand side of (66). This proves that we have $\sum_{j=0}^{4} \nu^{j} A_{j}\left(\partial_{\nu}, \partial_{s}, \partial_{\tau}\right) U_{n-j}(\nu, s, \tau)=0$. To sum up, we have verified that the functions $\left(u_{n}, U_{n}\right)$ that would be defined by means of (64)- 65) are also solution to (59). We conclude the proof by using Proposition 7.3

The previous proposition states that the definition of $u_{n}$ for a fixed $n \geq 0$ only requires the computation of auxiliary coefficients $\left[\ell_{\mathrm{N}}\left(\mathcal{N}_{j}^{k}\right)\right],\left[\ell_{\mathrm{N}}\left(\mathcal{U}_{j}^{k}\right)\right],\left[\ell_{\mathrm{D}}\left(\mathcal{U}_{j}^{k}\right)\right]$ and $\left[\ell_{\mathrm{D}}\left(\mathcal{N}_{j}^{k}\right)\right]$ which represent the effect of the periodic ring on the far field terms. This is interesting because it suggests to consider the following approximate transmission conditions

$$
\begin{aligned}
& {\left[\tilde{u}_{\delta}\right]_{\Gamma}=\sum_{j=1}^{n} \delta^{j}\left(\sum_{k=0}^{j}\left[\ell_{\mathrm{D}}\left(\mathcal{U}_{j}^{k}\right)\right] \partial_{\theta}^{k}\right)\left\langle\tilde{u}_{\delta}\right\rangle_{\Gamma}+\sum_{j=0}^{n-1} \delta^{j+1}\left(\sum_{k=0}^{j}\left[\ell_{\mathrm{D}}\left(\mathcal{N}_{j}^{k}\right)\right] \partial_{\theta}^{k}\right)\left\langle\partial_{r} \tilde{u}_{\delta}\right\rangle_{\Gamma},} \\
& {\left[\partial_{r} \tilde{u}_{\delta}\right]_{\Gamma}=\sum_{j=2}^{n+1} \delta^{j-1}\left(\sum_{k=0}^{j}\left[\ell_{\mathrm{N}}\left(\mathcal{U}_{j}^{k}\right)\right] \partial_{\theta}^{k}\right)\left\langle\tilde{u}_{\delta}\right\rangle_{\Gamma}+\sum_{j=1}^{n} \delta^{j}\left(\sum_{k=0}^{j}\left[\ell_{\mathrm{N}}\left(\mathcal{N}_{j}^{k}\right)\right] \partial_{\theta}^{k}\right)\left\langle\partial_{r} \tilde{u}_{\delta}\right\rangle_{\Gamma} .}
\end{aligned}
$$

where $\tilde{u}_{\delta}$ would be solution to the Helmholtz problem with such transmission conditions across the interface. It is not clear whether such a problem would be well posed though ; see [13] for the investigation of approximate transmission conditions associated with our problem.

\section{Definition of the global expansion}

The procedure of asymptotic analysis that we have been applying so far led to the definition of functions $u_{n}, U_{n}$ that provide the terms of the expansion of $u_{\delta}$ either "close" to the thin ring of inhomogeneities, or "far" from it. A global expansion can be constructed by interpolating those two expansions by means of a cut-off functions as follows (see Figure 3):

$$
\begin{aligned}
& \widetilde{u}_{N, \delta}(r, \theta)=\left[1-\chi\left(\frac{r-r_{*}}{\eta(\delta)}\right)\right] \sum_{n=0}^{N} \delta^{n} u_{n}(r, \theta)+\chi\left(\frac{r-r_{*}}{\eta(\delta)}\right) \sum_{n=0}^{N} \delta^{n} U_{n}\left(\frac{r-r_{*}}{\delta}, r_{*} \frac{\theta}{\delta}, \theta\right), \\
& \text { with } \eta(\delta)=\delta^{1-\frac{1}{N}} \quad \text { and } \quad \chi \in \mathscr{C}^{\infty}\left(\mathbb{R}_{+}\right), \text {and } \chi(\rho)=1 \text { for } \rho \leq 1, \quad \chi(\rho)=0 \text { for } \rho \geq 2 .
\end{aligned}
$$

We have to formulate two comments about such a formula. First of all, observe that according to the definition of $\chi$ we have $\widetilde{u}_{N, \delta}=\sum_{n=0}^{N} \delta^{n} u_{n}$ for $\left|r-r_{*}\right|>2 \eta(\delta)$, so Formula (67) takes the usual form of an expansion in $\delta$ except in a narrow region around the thin ring.

The second interesting comment concerns the near field terms in 67): we have to give a precise meaning to the truncated near field expansion $\sum_{n=0}^{N} \delta^{n} U_{n}\left(\frac{r-r_{*}}{\delta}, r_{*} \frac{\theta}{\delta}, \theta\right)$. Indeed, regarding the regularity $a$ priori of the functions $U_{n}$ that are only assumed to belong to $C^{\infty}\left(\mathbb{S}^{1}, \mathbb{V}_{+}^{1}(B)\right)$, it is not clear whether the expression " $U_{n}\left(\left(r-r_{*}\right) / \delta, r_{*} \theta / \delta, \theta\right)$ " makes sense, since $U_{n}(\nu, s, \tau)$ has been defined considering that $s$ and $\tau$ are separated variables. The following proposition shows the a priori regularity of $U_{n}$ actually allows to consider such a function.

\section{Proposition 9.1.}

Consider the immersion map $\phi_{\delta}: B \rightarrow B \times \mathbb{S}^{1}$ such that $\phi_{\delta}(r, \theta)=(\nu, s, \tau)$ where $\nu=\left(r-r_{*}\right) / \delta, s=$ $r_{*} \theta / \delta \bmod 2 \pi$ and $\tau=\theta$. It induces a unique continuous map $\phi_{\delta}^{*}: C_{\#}^{\infty}\left(\mathbb{S}^{1}, \mathrm{H}_{\mathrm{loc}, \#}^{k}(B)\right) \rightarrow \mathrm{H}_{\mathrm{loc}}^{k}(\Omega \backslash\{0\})$ for $k=0,1$ characterized by

$$
\left(\phi_{\delta}^{*} \varphi\right)(r, \theta)=\varphi\left(\phi_{\delta}(r, \theta)\right)=\varphi\left(\frac{r-r_{*}}{\delta}, r_{*} \frac{\theta}{\delta}, \theta\right) \quad \forall \varphi \in \mathscr{D}\left(B \times \mathbb{S}^{1}\right)
$$


Moreover, set $a>0, K_{a}:=\left\{\mathbf{x}(r, \theta)|| r-r_{*} \mid<a\right\}$ and $\left.B_{a}^{\delta}:=\right]-a / \delta, a / \delta\left[\times \mathbb{S}^{1}\right.$ : there exists a constant $C>0$ such that for any $a<r_{*} / 2$ we have

$$
\forall \varphi \in C^{\infty}\left(\mathbb{S}^{1}, \mathrm{~L}_{\text {loc }}^{2}(B)\right), \forall \delta \in(0,1), \quad\left\|\phi_{\delta}^{*} \varphi\right\|_{\mathrm{L}^{2}\left(K_{a}\right)}^{2} \leq C \delta .
$$

Proof. Note that $\mathscr{D}_{\#}\left(B \times \mathbb{S}^{1}\right)$ is dense in $\mathrm{H}_{\#}^{1}\left(\mathbb{S}^{1}, \mathrm{~L}_{\text {loc }}^{2}(B)\right)$ (the proof is very close to the proof of Lemma 11.3 so it is left to the reader). Relying on this density argument, it suffices to prove that for any $0<a<r_{*}$, there exists a constant $C_{a}>0$ such that

$$
\left\|\phi_{\delta}^{*} \varphi\right\|_{\mathrm{L}^{2}\left(K_{a}\right)}^{2} \leq C_{a}\|\varphi\|_{\mathrm{H}^{1}\left(\mathbb{S}^{1}, \mathrm{~L}^{2}\left(B_{a}^{\delta}\right)\right)}^{2} \quad \forall \varphi \in \mathscr{D}_{\#}\left(B \times \mathbb{S}^{1}\right) .
$$

For any $\varphi \in \mathscr{D}\left(B \times \mathbb{S}^{1}\right)$ we have

$$
\left(\phi_{\delta}^{*} \varphi\right)(r, \theta)=\varphi\left(\frac{r-r_{*}}{\delta}, r_{*} \frac{\theta}{\delta}, 0\right)+\int_{0}^{\theta} \partial_{\tau} \varphi\left(\frac{r-r_{*}}{\delta}, r_{*} \frac{\theta}{\delta}, \tau\right) d \tau .
$$

Consequently, introducing the usual change of variables $\nu=\left(r-r_{*}\right) / \delta, s=r_{*} \theta / \delta$ and using the periodicity of $\varphi$ with respect to $s$, we get

$$
\left\|\phi_{\delta}^{*} \varphi\right\|_{\left.\mathrm{L}^{2}\left(K_{a}\right)\right)}^{2} \leq 2\left(a+r_{*}\right) \frac{\delta^{2}}{r_{*}} N_{\delta}\|\varphi(\cdot, 0)\|_{\mathrm{L}^{2}\left(B_{a}^{\delta}\right)}^{2}+2\left(a+r_{*}\right) \frac{\delta^{2}}{r_{*}} N_{\delta} \int_{0}^{2 \pi}\left\|\partial_{\tau} \varphi(\cdot, \tau)\right\|_{\mathrm{L}^{2}\left(B_{a}^{\delta}\right)}^{2} d \tau,
$$

where $N_{\delta}=2 \pi r_{*} / \delta$ is the number of periodicity cells in the periodic ring (recall that, according to the general assumptions stated in Section 1 we have $N_{\delta} \in \mathbb{N}$ ). There only remains to estimate $\|\varphi(\cdot, 0)\|_{\mathrm{L}^{2}\left(B_{a}^{\delta}\right)}$ in order to conclude. As $\tau \mapsto\|\varphi(\cdot, \tau)\|_{\mathrm{L}^{2}\left(B_{a}^{\delta}\right)}$ is in $\mathrm{H}^{1}\left(\mathbb{S}^{1}\right)$, it suffices to apply the trace theorem for $\mathrm{H}^{1}\left(\mathbb{S}^{1}\right)$. The continuity estimates $(68)$ is then obtained. Note in addition that this estimate holds as well if $a$ depends on $\delta$ in such a way that $a_{\delta} \rightarrow 0$ when $\delta \rightarrow 0$. Later on during the error analysis, we shall be particularly interested in the case where $a_{\delta}=\eta(\delta)$.

This proposition shows that (67) indeed makes sense and that the near field terms should be understood in the sense of " $\phi_{\delta}^{*} U_{n}$ ". Moreover, using density arguments, the usual formula for the derivatives of a composition of functions holds, namely $\forall \varphi \in C_{\#}^{\infty}\left(\mathbb{S}^{1}, \mathrm{H}_{\mathrm{loc}}^{1}(B)\right)$

$$
\phi_{\delta}^{*}\left(\frac{1}{\delta} \partial_{\nu} g\right):=\partial_{r}\left(\phi_{\delta}^{*} g\right) \text { and } \phi_{\delta}^{*}\left(\frac{r_{*}}{\delta} \partial_{s} g+\partial_{\tau} g\right):=\partial_{\theta}\left(\phi_{\delta}^{*} g\right) \text {. }
$$

\section{Error estimates (Step V)}

Now we have defined the terms $u_{n}, U_{n}$ solution to Problem (59), and constructed a global expansion through 677. Note however that, for the moment, the expansion (67) has only the status of a "good candidate" for being a sharp approximation to $u_{\delta}$. In this section, we are going to prove that, it is indeed a strong approximation by providing error estimates. To be more precise we are going to prove the following result

Theorem 10.1. Let $u_{\delta}$ be the solution of Problem (5), and $\widetilde{u}_{N, \delta}$ be defined by Formula (67). For any $N \geq 0$, there exists $C_{N}>0$ independent of $\delta$ (but a priori depending on $N$ ) such that

$$
\left\|u_{\delta}-\widetilde{u}_{N, \delta}\right\|_{\mathrm{H}^{1}(\Omega)} \leqslant C_{N} \delta^{N-2} \quad \forall \delta \in(0,1) .
$$

As a byproduct, such an estimate yields optimal error bounds for the far field expansion in any region that excludes a neighborhood of the thin ring:

Theorem 10.2. Let $\gamma \in\left(0, \gamma_{*}\right)$ and $\mathcal{O}=\left\{\mathbf{x}(r, \theta) \in \Omega|| r-r_{*} \mid>\gamma\right\}$. Then, for any $N \geq 0$, there exists $C_{N}>0$ independent of $\delta$ such that

$$
\left\|u_{\delta}-\sum_{n=0}^{N} \delta^{n} u_{n}\right\|_{\mathrm{H}^{1}(\mathcal{O})} \leq C_{N} \delta^{N+1}
$$


Proof. Theorem 10.2 results from a direct application of Theorem 10.1 and the triangular inequality:

$$
\left\|u_{\delta}-\sum_{n=0}^{N} \delta^{n} u_{n}\right\|_{\mathrm{H}^{1}(\mathcal{O})} \leq\left\|u_{\delta}-\widetilde{u}_{N+3, \delta}\right\|_{\mathrm{H}^{1}(\mathcal{O})}+\delta^{N+1} \sum_{k=0}^{2} \delta^{k}\left\|u_{N+k+1}\right\|_{\mathrm{H}^{1}(\mathcal{O})}=O\left(\delta^{N+1}\right) .
$$

\subsection{Stability}

As is standard in asymptotic analysis, the first step in the proof of Theorem 10.1 consists in establishing that the solution to Problem (5) continuously depends on the data, with a continuity constant that remains bounded as $\delta \rightarrow 0$.

\section{Proposition 10.3.}

Problem (5) is well-posed. Moreover, there exist two positive constants $C$ and $\delta_{0}$ independent of $\delta$ such that,

$$
\|u\|_{\mathrm{H}^{1}(\Omega)} \leq C \sup _{v \in \mathrm{H}^{1}(\Omega) \backslash\{0\}} \frac{\left|a_{\delta}(u, v)\right|}{\|v\|_{\mathrm{H}^{1}(\Omega)}} \quad \forall u \in \mathrm{H}^{1}(\Omega), \quad \forall \delta \in\left(0, \delta_{0}\right) .
$$

Proof. The well posedness of Problem (5) is a classical result, see for example [24]. In order to prove estimate (71), we proceed by contradiction which is a standard approach (see for instance theorem 2.1 of [16], lemma 12.14 of [32] or theorem 1.2.1 of [10] for similar proofs). Assume that (71) does not hold. Then there exists a sequence $\delta_{n}$ which tends to 0 and a sequence $u_{n} \in \mathrm{H}^{1}(\Omega), n \in \mathbb{N}$ such that

$$
\left\|u_{n}\right\|_{H^{1}(\Omega)}=1 \quad(a) \quad \text { and } \quad \lim _{n \rightarrow \infty} \sup _{v \in H^{1}(\Omega), v \neq 0} \frac{\left|a_{\delta_{n}}\left(u_{n}, v\right)\right|}{\|v\|_{\mathrm{H}^{1}(\Omega)}}=0
$$

The sequence $u_{n}$ is bounded in $\mathrm{H} 1(\Omega)$ so, introducing a subsequence if necessary, we may assume that it converges toward a function $u \in \mathrm{H}_{1}(\Omega)$ weakly in $\mathrm{H} 1(\Omega)$ and strongly in $L^{2}(\Omega)$. Moreover, for any $v \in \mathrm{H}^{1}(\Omega), \lim _{n \rightarrow+\infty}\left\|\left(\epsilon_{\delta_{n}}^{-1}-\epsilon_{\infty}^{-1}\right) \nabla v\right\|_{L^{2}(\Omega)}=0$ and $\lim _{n \rightarrow+\infty}\left\|\left(\mu_{\delta_{n}}-\mu_{\infty}\right) v\right\|_{L^{2}(\Omega)}=0$, since $\epsilon_{\delta_{n}}$ and $\mu_{\delta_{n}}$ actually converge to $\epsilon_{\infty}$ and $\mu_{\infty}$ almost everywhere in $\Omega$. As a consequence

$$
\lim _{n \rightarrow+\infty} a_{\delta_{n}}\left(u_{n}, v\right)=a_{0}(u, v),
$$

where $a_{0}(u, v)=\int_{\Omega}\left(\epsilon_{\infty}^{-1} \nabla u \cdot \nabla \bar{v}-\omega^{2} \mu_{\infty} u \bar{v}\right) d \mathbf{x}+\int_{\partial \Omega} \bar{v} T u d \sigma$. Combining (72) and the assumption (b), we have $a_{0}(u, v)=0$ for any $v \in \mathrm{H}^{1}(\Omega)$. It exactly means that $u$ is an outgoing wave which solves the homogeneous Helmholtz equation in $\Omega$. We thus get $u=0$ (see for instance [11] or [25]) and consequently $\lim _{n \rightarrow+\infty}\left\|u_{n}\right\|_{L^{2}(\Omega)}=0$. Also, since $u_{n}$ satisfies the Helmholtz equation, it is easy to check that

$$
\mathcal{R} e\left\{a_{\delta_{n}}\left(u_{n}, u_{n}\right)\right\}=\int_{\Omega} \epsilon_{\delta_{n}}\left|\nabla u_{n}\right|^{2} d \mathbf{x}+\mathcal{R} e\left\{\int_{\partial \Omega} \bar{u}_{n} T u_{n} d \sigma\right\}-\omega^{2} \int_{\Omega} \mu_{\delta_{n}}\left|u_{n}\right|^{2} d \mathbf{x} .
$$

But, it is well known (see for instance [1]) that $\mathcal{R} e\left\{\int_{\partial \Omega} \bar{u}_{n} T u_{n} d \sigma\right\} \geq \frac{1}{2 r_{e}} \int_{\partial \Omega}\left|u_{n}\right|^{2}$. Then,

$$
\left\|\nabla u_{n}\right\|_{\mathrm{L}^{2}(\Omega)}^{2}+\frac{1}{2 r_{e}} \int_{\partial \Omega}\left|u_{n}\right|^{2} d \sigma \leq C\left(\left|a_{\delta_{n}}\left(u_{n}, u_{n}\right)\right|+\left\|u_{n}\right\|_{\mathrm{L}^{2}(\Omega)}^{2}\right) \quad \forall n \geq 0 .
$$

for a constant $C>0$ independent of $n$, chosen larger than $\omega^{2} \sup _{\mathbb{R}^{2}}|\mu| / 2$ Letting $n \rightarrow+\infty$, we obtain $\lim _{n \rightarrow \infty}\left\|u_{n}\right\|_{\mathrm{H}^{1}(\Omega)}=0$, which contradicts the assumption $(a)$.

\subsection{Consistency}

The second step in the error analysis consists in applying the stability estimate to the difference $u_{\delta}-\widetilde{u}_{N, \delta}$. As a consequence, according to Proposition 10.3, it suffices to provide estimates for expressions of the 
form $a_{\delta}\left(u_{\delta}-\widetilde{u}_{N, \delta}, v\right)$ in order to obtain a bound for $\left\|u_{\delta}-\widetilde{u}_{N, \delta}\right\|_{\mathrm{H}^{1}(\Omega)}$. Let us introduce the function $\chi_{\delta}(\mathbf{x})=\chi\left(\left(r-r_{*}\right) / \eta(\delta)\right)$ and observe that

$$
a_{\delta}\left(\chi_{\delta} u, v\right)=a_{\delta}\left(u, \chi_{\delta} v\right)+\epsilon_{\infty}^{-1} \int_{\Omega}\left(\nabla \bar{v} \cdot \nabla \chi_{\delta}\right) u d \mathbf{x}-\epsilon_{\infty}^{-1} \int_{\Omega}\left(\bar{v} \nabla \chi_{\delta}\right) \cdot \nabla u d \mathbf{x} \quad \forall u, v \in \mathrm{H}^{1}(\Omega) .
$$

Now take an arbitrary $v \in \mathrm{H}^{1}(\Omega)$, and plug Formula (67) into $a_{\delta}\left(u_{\delta}-\widetilde{u}_{N, \delta}, v\right)$. Taking into account (73) we obtain

$$
\begin{aligned}
a_{\delta}\left(\widetilde{u}_{N, \delta}-u_{\delta}, v\right)=-\int_{\Omega} f \bar{v} d \mathbf{x} & +\sum_{n=0}^{N} \delta^{n} a_{\delta}\left(u_{n},\left(1-\chi_{\delta}\right) v\right)+\sum_{n=0}^{N} \delta^{n} a_{\delta}\left(\phi_{\delta}^{*} U_{n}, \chi_{\delta} v\right) \\
& -\sum_{n=0}^{N} \delta^{n} \int_{\Omega}\left(\nabla \bar{v} \cdot \nabla \chi_{\delta}\right)\left(u_{n}-\phi_{\delta}^{*} U_{n}\right) d \mathbf{x} \\
& +\sum_{n=0}^{N} \delta^{n} \int_{\Omega}\left(\bar{v} \nabla \chi_{\delta}\right) \cdot \nabla\left(u_{n}-\phi_{\delta}^{*} U_{n}\right) d \mathbf{x} .
\end{aligned}
$$

The first two terms in the right hand side above compensate each other. Indeed note that, since $\chi_{\delta} v$ vanishes in the neighborhood of $\Gamma$, we have $a_{\delta}\left(u_{n}, \chi_{\delta} v\right)=a_{0}\left(u_{n}, \chi_{\delta} v\right), \forall n \geqslant 0, \forall \delta \in(0,1), \forall v \in \mathrm{H}^{1}(\Omega)$ where $a_{0}(u, v)=\int_{\Omega} \epsilon_{\infty}^{-1} \nabla u \cdot \nabla \bar{v} d \mathbf{x}-\int_{\Omega} \omega^{2} \mu_{\infty} u \bar{v} d \mathbf{x}+\int_{\partial \Omega} \bar{v} T u d \sigma$. Besides, according to the definition of $u_{n}$ given by [59), we have $-\int_{\Omega} f \bar{v} d \mathbf{x}+a_{0}\left(u_{0}, \chi_{\delta} v\right)=0$ and $a_{0}\left(u_{n}, \chi_{\delta} v\right)=0$. As a consequence, (74) reduces to

$$
\begin{aligned}
a_{\delta}\left(\widetilde{u}_{N, \delta}-u_{\delta}, v\right)= & \sum_{n=0}^{N} \delta^{n} \int_{\Omega}\left(\nabla \bar{v} \cdot \nabla \chi_{\delta}\right)\left(u_{n}-\phi_{\delta}^{*} U_{n}\right) d \mathbf{x} \\
& -\sum_{n=0}^{N} \delta^{n} \int_{\Omega}\left(\bar{v} \nabla \chi_{\delta}\right) \cdot \nabla\left(u_{n}-\phi_{\delta}^{*} U_{n}\right) d \mathbf{x} \\
& +\sum_{n=0}^{N} \delta^{n} a_{\delta}\left(\phi_{\delta}^{*} U_{n}, \chi_{\delta} v\right) .
\end{aligned}
$$

The first two terms in the right-hand side above will be called matching error: it measures the mismatch between the far and near field expansions in the overlapping areas. The last term will be called near field error. It measures how much the near field truncated expansion fails to satisfy the original Helmholtz expansion. In the two following technical parts we shall provide a suitable upper bound for each of these three terms.

\subsection{Matching error}

In this paragraph we provide a bound for the first two terms in the right-hand side of (74). We present the derivation of such a bound only for the first term, since the derivation of a bound for the second is nearly the same. Let us introduce the set $\mathcal{T}_{\delta}=\left\{\mathbf{x}(r, \theta)|\eta(\delta)<| r-r_{*} \mid<2 \eta(\delta)\right\}$, and observe that $\operatorname{supp}\left(\chi_{\delta}\right) \subset \mathcal{T}_{\delta}$. Applying Cauchy-Schwarz inequality yields

$$
\left|\sum_{n=0}^{N} \delta^{n} \int_{\Omega}\left(\nabla \bar{v} \cdot \nabla \chi_{\delta}\right)\left(u_{n}-\phi_{\delta}^{*} U_{n}\right) d \mathbf{x}\right| \leq\|v\|_{\mathrm{H}^{1}(\Omega)}\left\|\nabla \chi_{\delta}\right\|_{\mathrm{L}^{\infty}(\mathbb{R})}\left\|\sum_{n=0}^{N} \delta^{n}\left(u_{n}-\phi_{\delta}^{*} U_{n}\right)\right\|_{\mathrm{L}^{2}\left(\mathcal{T}_{\delta}\right)} .
$$

Observe that $\left\|\nabla \chi_{\delta}\right\|_{L^{\infty}(\mathbb{R})} \leq \delta^{-1}\|\nabla \chi\|_{L^{\infty}(\mathbb{R})}$. We want to obtain an upper bound for $\| \sum_{n=0}^{N} \delta^{n}\left(u_{n}-\right.$ $\left.\phi_{\delta}^{*} U_{n}\right) \|_{\mathrm{L}^{2}\left(\mathcal{T}_{\delta}\right)}$. Let us denote $\mathcal{T}_{\delta}^{ \pm}=\left\{\mathbf{x} \in \mathcal{T}_{\delta}| \pm| \mathbf{x} \mid>r_{*}\right\}$ so that we have $\mathcal{T}_{\delta}=\mathcal{T}_{\delta}^{+} \cup \mathcal{T}_{\delta}^{-}$. We study $\sum_{n} \delta^{n}\left(u_{n}-\phi_{\delta}^{*} U_{n}\right)$ first in $\mathcal{T}_{\delta}^{+}$where we have

$$
\frac{\eta(\delta)}{\delta}<\nu<2 \frac{\eta(\delta)}{\delta} \quad \text { when } \quad \mathbf{x}(r, \theta) \in \mathcal{T}_{\delta}^{+} \quad \text { with } \quad \nu=\frac{r-r_{*}}{\delta}
$$


As a consequence $\nu \rightarrow+\infty$ when $\mathbf{x} \in \mathcal{T}_{\delta}^{+}$. According to (59), Proposition 5.2 can be applied to $U_{0}, \ldots, U_{N}$. As a consequence there exists a family $\mathfrak{U}_{0}, \ldots, \mathfrak{U}_{N}$ belonging to $C^{\infty}\left(\mathbb{S}^{1}, \mathbb{V}_{-}^{1}(B)\right)$ such that for $\mathbf{x}(r, \theta) \in \mathcal{T}_{\delta}^{+}$we have

$$
\begin{aligned}
\sum_{n=0}^{N} \delta^{n} \phi_{\delta}^{*} U_{n}=\sum_{n=0}^{N} \delta^{n} \phi_{\delta}^{*}\left[\sum_{k=0}^{n+1} \nu^{k}\left(s_{k}^{0}\left(\partial_{\tau}\right) \cdot \ell_{\mathrm{D}}^{+}\left(U_{n-k}\right)+s_{k}^{1}\left(\partial_{\tau}\right) \cdot \ell_{\mathrm{N}}^{+}\left(U_{n+1-k}\right)\right)+\mathfrak{U}_{n}\right] \\
=\sum_{n=0}^{N} \sum_{k=0}^{n+1} \delta^{n}\left(\frac{r-r_{*}}{\delta}\right)^{k}\left(s_{k}^{0}\left(\partial_{\theta}\right) \cdot \ell_{\mathrm{D}}^{+}\left(U_{n-k}\right)+s_{k}^{1}\left(\partial_{\theta}\right) \cdot \ell_{\mathrm{N}}^{+}\left(U_{n+1-k}\right)\right)+\sum_{n=0}^{N} \delta^{n} \phi_{\delta}^{*} \mathfrak{U}_{n} .
\end{aligned}
$$

In the first line $\ell_{\mathrm{D}}^{+}\left(U_{n-k}\right)$ should be understood as $\ell_{\mathrm{D}}^{+}\left(U_{n-k}(\cdot, \tau)\right)$, whereas it should be understood as $\ell_{\mathrm{D}}^{+}\left(U_{n-k}(\cdot, \theta)\right)$ in the second line. Let us rearrange the first sum in the right-hand side above, using the change of indices $p=n-k, q=k$ and taking the matching conditions (54) into account:

$$
\begin{aligned}
\sum_{n=0}^{N} \sum_{k=0}^{n+1} \delta^{n}( & \left.\frac{r-r_{*}}{\delta}\right)^{k}\left(s_{k}^{0}\left(\partial_{\theta}\right) \cdot \ell_{\mathrm{D}}^{+}\left(U_{n-k}\right)+s_{k}^{1}\left(\partial_{\theta}\right) \cdot \ell_{\mathrm{N}}^{+}\left(U_{n+1-k}\right)\right) \\
& =\sum_{p=-1}^{N} \sum_{q=0}^{N-p} \delta^{p}\left(r-r_{*}\right)^{q}\left(s_{k}^{0}\left(\partial_{\theta}\right) \cdot \ell_{\mathrm{D}}^{+}\left(U_{p}\right)+s_{k}^{1}\left(\partial_{\theta}\right) \cdot \ell_{\mathrm{N}}^{+}\left(U_{p+1}\right)\right), \\
& =\sum_{p=0}^{N} \sum_{q=0}^{N-p} \delta^{p}\left(r-r_{*}\right)^{q}\left(\left.s_{k}^{0}\left(\partial_{\theta}\right) \cdot u_{p}\right|_{\Gamma} ^{+}+\left.s_{k}^{1}\left(\partial_{\theta}\right) \cdot \partial_{r} u_{p}\right|_{\Gamma} ^{+}\right) .
\end{aligned}
$$

In the calculus above we used our convention that $U_{n}=0$ for $n \leq 0$, and we also used that $s_{0}^{1}\left(\partial_{\theta}\right)=0$ by definition. Now according to the definition of $u_{n}$ given by (59), Proposition 2.1 can be applied to each $u_{n}$ and yields (19), which can be plugged into the last sum in (78). This gives

$$
\begin{aligned}
\sum_{p=0}^{N} \sum_{q=0}^{N-p} \delta^{p}\left(r-r_{*}\right)^{q} & \left(\left.s_{k}^{0}\left(\partial_{\theta}\right) \cdot u_{p}\right|_{\Gamma} ^{+}+\left.s_{k}^{1}\left(\partial_{\theta}\right) \cdot \partial_{r} u_{p}\right|_{\Gamma} ^{+}\right) \\
& =\sum_{p=0}^{N} \delta^{n} u_{n}-\sum_{n=0}^{N} \delta^{n}\left(r-r_{*}\right)^{N+1-n} \mathfrak{u}_{n, N-n}(r, \theta),
\end{aligned}
$$

where $\mathfrak{u}_{0, N}, \mathfrak{u}_{1, N-1}, \ldots, \mathfrak{u}_{N, 0} \in \mathrm{H}^{1}\left(\Omega_{ \pm}\right)$do not depend on $\delta$. The same calculus as for $\mathcal{T}_{\delta}^{+}$can be achieved for $\mathcal{T}_{\delta}^{-}$. Combining equations (77), (78), (79) both for $\mathcal{T}_{\delta}^{+}$and $\mathcal{T}_{\delta}^{-}$, and taking into account that $\eta(\delta)<\left|r-r_{*}\right|<2 \eta(\delta)$ inside $\mathcal{T}_{\delta}$, we obtain

$$
\begin{array}{r}
\left\|\sum_{n=0}^{N} \delta^{n}\left(u_{n}-\phi_{\delta}^{*} U_{n}\right)\right\|_{\mathcal{L}^{2}\left(\mathcal{T}_{\delta}\right)}=\left\|\sum_{n=0}^{N} \delta^{n}\left(r-r_{*}\right)^{N+1-n} \mathfrak{u}_{n, N-n}-\sum_{n=0}^{N} \delta^{n} \phi_{\delta}^{*} \mathfrak{U}_{n}\right\|_{\mathrm{L}^{2}\left(\mathcal{T}_{\delta}\right)}, \\
\leq 2^{N+1} \sum_{n=0}^{N} \delta^{n} \eta(\delta)^{N+1-n}\left\|\mathfrak{u}_{n, N-n}\right\|_{\mathrm{L}^{2}(\Omega)}+\sum_{n=0}^{N} \delta^{n}\left\|\phi_{\delta}^{*} \mathfrak{U}_{n}\right\|_{\mathrm{L}^{2}\left(\mathcal{T}_{\delta}\right)} .
\end{array}
$$

To treat the last terms in the inequality above, recall that each $\mathfrak{U}_{n}$ is "evanescent at infinity". Observe that $\mathcal{T}_{\delta} \subset K_{a_{\delta}}=\left\{\mathbf{x}(r, \theta) \quad|| r-r_{*} \mid<a_{\delta}\right\}$ for $a_{\delta}=2 \eta(\delta)$, so we can apply Estimate (68): there exists a constant $C>0$ independent of $\delta$ such that, $\forall \delta \in(0,1)$, we have

$$
\begin{aligned}
\left\|\phi_{\delta}^{*} \mathfrak{U}_{n}\right\|_{\mathrm{L}^{2}\left(\mathcal{T}_{\delta}\right)}^{2} & \leqslant e^{-1 / \eta(\delta)}\left\|\phi_{\delta}^{*}\left(e^{\frac{|\nu|}{2}} \mathfrak{U}_{n}\right)\right\|_{\mathrm{L}^{2}\left(\mathcal{T}_{\delta}\right)}^{2} \leqslant C \delta e^{-1 / \eta(\delta)}\left\|e^{\frac{|\nu|}{2}} \mathfrak{U}_{n}\right\|_{\mathrm{H}^{1}\left(\mathbb{S}^{1}, \mathrm{~L}^{2}\left(B_{a}^{\delta}\right)\right)}^{2}, \\
& \leqslant C \delta e^{-1 / \eta(\delta)} \int_{0}^{2 \pi}\left\|\mathfrak{U}_{n}(\cdot, \tau)\right\|_{\mathbb{V}_{-}^{0}(B)}^{2}+\left\|\partial_{\tau} \mathfrak{U}_{n}(\cdot, \tau)\right\|_{\mathbb{V}_{-}^{0}(B)}^{2} d \tau
\end{aligned}
$$

Besides $\delta e^{-1 / \eta(\delta)}=O\left(\delta^{2 N}\right)$ since $\eta(\delta)=\delta^{1-1 / N}$. Let us plug (81) into (80). Taking into account in addition that $\left\|\mathfrak{u}_{n, N-n}\right\|_{\mathrm{L}^{2}(\Omega)}$ is independent of $\delta$, and that $\delta^{n} \eta(\delta)^{N+1-n}=O\left(\delta^{N}\right)$ since $\eta(\delta)=\delta^{1-1 / N}$, 
we obtain the existence of a constant $C>0$ independent of $\delta$ such that

$$
\left\|\sum_{p=0}^{N} \delta^{n}\left(u_{n}-\phi_{\delta}^{*} U_{n}\right)\right\|_{\mathrm{L}^{2}\left(\mathcal{T}_{\delta}\right)} \leq C \delta^{N} \quad \forall \delta \in(0,1) .
$$

Of course the constant $C$ depends on $N$ and on $u_{n}, U_{n}, n=0, \ldots, N$. It also depends on $r_{*}$. Coming back to (76), we see that Inequality (82) leads to an error bound for the first term in the right-hand side of (74). A similar bound can be derived for the second term using the same approach. We summarize both estimates in the following proposition.

\section{Proposition 10.4 (Estimate for the matching error).}

For a given $N \in \mathbb{N}$, let $\left(u_{0}, U_{0}\right), \ldots\left(u_{n}, U_{n}\right)$ be the unique solutions to Problems (57)-(58)-(59). Let $\chi_{\delta}(\mathbf{x})=\chi\left(\left(r-r_{*}\right) / \eta(\delta)\right)$ where $\chi$ and $\eta(\delta)$ are defined in (67). Then there exists a constant $C, \delta_{0}>0$ independent of $\delta$ (depending on $N$ though) such that

$$
\begin{aligned}
&\left|\sum_{n=0}^{N} \delta^{n} \int_{\Omega}\left(\nabla \bar{v} \cdot \nabla \chi_{\delta}\right)\left(u_{n}-\phi_{\delta}^{*} U_{n}\right) d \mathbf{x}-\sum_{n=0}^{N} \delta^{n} \int_{\Omega}\left(\bar{v} \nabla \chi_{\delta}\right) \cdot \nabla\left(u_{n}-\phi_{\delta}^{*} U_{n}\right) d \mathbf{x}\right| \\
& \leq C\|v\|_{\mathrm{H}^{1}(\Omega)} \delta^{N-1} \quad \forall v \in \mathrm{H}^{1}(\Omega), \quad \forall \delta \in\left(0, \delta_{0}\right) .
\end{aligned}
$$

\subsection{Near field error}

In order to prove Theorem 10.1 the only remaining step consists in estimating the last term of (74), i.e,

$$
\sum_{n=0}^{N} \delta^{n} a_{\delta}\left(\phi_{\delta}^{*} U_{n}, \chi_{\delta} v\right)
$$

As we have already said, this term does not vanish since the near field truncated expansion does not satisfy exactly the original homogeneous Helmholtz equation (see 30). To obtain an upper-bound, we would like to use the near field terms equations (30) which are contained (in a variational way) in (59). However, it is not possible to make direct use of these equations since the quantity which appears in (74) is not $U_{n}$ but $\phi_{\delta}^{*} U_{n}$. The next proposition will be used to bypass this difficulty and can be seen as an extension of Proposition 9.1

\section{Proposition 10.5.}

The map $\phi_{\delta}^{*}: C^{\infty}\left(\mathbb{S}^{1}, \mathrm{~L}_{\mathrm{loc}}^{2}(B)\right) \rightarrow \mathrm{L}_{\mathrm{loc}}^{2}(\Omega \backslash\{0\})$ defined in Proposition 9.1 can be extended in a unique manner as a continuous map $\phi_{\delta}^{*}: C^{\infty}\left(\mathbb{S}^{1}, \mathrm{H}_{\mathrm{loc}}^{1}(B)^{\prime}\right) \rightarrow \mathrm{H}_{\mathrm{loc}}^{1}(\Omega \backslash\{0\})^{\prime}$ characterized by the following identities: $\forall g \in C^{\infty}\left(\mathbb{S}^{1}, \mathrm{~L}_{\text {loc }}^{2}(B)\right)$ we have

$$
\phi_{\delta}^{*}\left(\frac{1}{\delta} \partial_{\nu} g\right):=\partial_{r}\left(\phi_{\delta}^{*} g\right) \quad \text { and } \quad \phi_{\delta}^{*}\left(\frac{r_{*}}{\delta} \partial_{s} g+\partial_{\tau} g\right):=\partial_{\theta}\left(\phi_{\delta}^{*} g\right) \text {. }
$$

As an extension, the map $\phi_{\delta}^{*}$ of Proposition 10.5 coincides over $C^{\infty}\left(\mathbb{S}^{1}, \mathrm{~L}_{\text {loc }}^{2}(B)\right)$ with the map defined by Proposition 9.1. Besides the relations (83) should be understood in the weak sense, namely (for $g \in$ $\left.C^{\infty}\left(\mathbb{S}^{1}, \mathrm{~L}_{\text {loc }}^{2}(B)\right)\right)$

$$
\begin{aligned}
\left\langle\phi_{\delta}^{*}\left(\delta^{-1} \partial_{\nu} g\right), v\right\rangle_{\Omega} & =-\int_{\Omega}\left(\phi_{\delta}^{*} g\right)(\mathbf{x}) \partial_{r}(\overline{v r}(\mathbf{x})) / r d \mathbf{x} \\
\left\langle\phi_{\delta}^{*}\left(r_{*} \delta^{-1} \partial_{s} g+\partial_{\tau} g\right), v\right\rangle_{\Omega} & =-\int_{\Omega}\left(\phi_{\delta}^{*} g\right)(\mathbf{x}) \partial_{\theta} \bar{v}(\mathbf{x}) d \mathbf{x} \quad \forall v \in \mathscr{D}(\Omega \backslash\{0\}),
\end{aligned}
$$

where $<,>_{\Omega}$ refers to the duality pairing between $\mathscr{D}(\Omega \backslash\{0\})$ and $\mathscr{D}^{\prime}(\Omega \backslash\{0\})$. Identities 84 are a weak counterpart of Identities (21). These properties of the map $\phi_{\delta}^{*}$ can be used for studying the third term 
in (74). Observe that, in (74), we have $\operatorname{supp}\left\{\chi_{\delta} v\right\} \subset \Omega \backslash\{0\}$, so we have

$$
\begin{aligned}
\sum_{n=0}^{N} \delta^{n} a_{\delta}\left(\phi_{\delta}^{*} U_{n}, \chi_{\delta} v\right)= & \sum_{n=0}^{N} \delta^{n} \int_{\Omega} \epsilon_{\delta}^{-1} \partial_{r}\left(\phi_{\delta}^{*} U_{n}\right) \partial_{r} \overline{\left(\chi_{\delta} v\right)} d \mathbf{x} \\
& +\sum_{n=0}^{N} \delta^{n} \int_{\Omega} \epsilon_{\delta}^{-1} r^{-2} \partial_{\theta}\left(\phi_{\delta}^{*} U_{n}\right) \partial_{\theta} \overline{\left(\chi_{\delta} v\right)} d \mathbf{x} \\
& -\sum_{n=0}^{N} \delta^{n} \int_{\Omega} \omega^{2} \mu_{\delta}\left(\phi_{\delta}^{*} U_{n}\right) \overline{\left(\chi_{\delta} v\right)} d \mathbf{x} .
\end{aligned}
$$

Let us study the first integral in the right hand side above. Note that $\epsilon_{\delta}^{-1}=\phi_{\delta}^{*}\left(\epsilon^{-1}\right)$, and that $U_{n} \in$ $C_{\#}^{\infty}\left(\mathbb{S}^{1}, \mathbb{V}_{+}^{1}(B)\right)$ by construction. Using the identities $[\mathbf{8 3}$, we obtain

$$
\int_{\Omega} \epsilon_{\delta}^{-1} \partial_{r}\left(\phi_{\delta}^{*} U_{n}\right) \partial_{r} \overline{\left(\chi_{\delta} v\right)} d \mathbf{x}=\left\langle\delta^{-2} \phi_{\delta}^{*}\left(\left(r_{*}+\delta \nu\right) \partial_{\nu}\left(\epsilon^{-1}\left(r_{*}+\delta \nu\right) \partial_{\nu} U_{n}\right)\right), \frac{\chi_{\delta} v}{r^{2}}\right\rangle_{\Omega} .
$$

A similar identity can be derived for the second and third integral in the right hand side of 85 ). Gathering all three expressions obtained in this manner leads to a calculus similar to (22). Indeed, according to the definition of $A_{j}$ given by (24)-(25), we obtain the following equality

$$
\begin{aligned}
\sum_{n=0}^{N} \delta^{n} a_{\delta}\left(\phi_{\delta}^{*} U_{n}, \chi_{\delta} v\right) & =\sum_{n=0}^{N} \delta^{n}\left\langle\phi_{\delta}^{*}\left((\nu \delta)^{-2} \sum_{j=0}^{4}(\delta \nu)^{j} A_{j} U_{n}\right), \frac{\chi_{\delta} v}{r^{2}}\right\rangle_{\Omega} \\
& =\left\langle\phi_{\delta}^{*}\left((\nu \delta)^{-2} \sum_{n=0}^{N} \sum_{j=0}^{4} \delta^{n+j} \nu^{j} A_{j} U_{n}\right), \frac{\chi_{\delta} v}{r^{2}}\right\rangle_{\Omega}
\end{aligned}
$$

Thanks to Equation (30) contained in (59), many terms in the right hand side of (86) cancel. Indeed, using the change of index $p=n+j$ we obtain

$$
\sum_{n=0}^{N} \sum_{j=0}^{4} \delta^{n+j} \nu^{j} A_{j} U_{n}=\sum_{p=0}^{N} \underbrace{\left(\sum_{j=0}^{4} \delta^{p} \nu^{j} A_{j} U_{p-j}\right)}_{=0 \text { according to [30 }}+\sum_{n=N-3}^{N} \sum_{j=N+1-n}^{4} \delta^{n+j} \nu^{j} A_{j} U_{n} .
$$

Plugging this into Equation (86) yields

$$
\sum_{n=0}^{N} \delta^{n} a_{\delta}\left(\phi_{\delta}^{*} U_{n}, \chi_{\delta} v\right)=\sum_{n=N-3}^{N} \sum_{j=N+1-n}^{4} \delta^{n+j-2}\left\langle\phi_{\delta}^{*}\left(\nu^{j-2} A_{j} U_{n}\right), \frac{\chi_{\delta} v}{r^{2}}\right\rangle_{\Omega} .
$$

Note that in the sum of the right-hand side above, we have $j+n \geq N+1$. As a consequence

$$
\left|\sum_{n=0}^{N} \delta^{n} a_{\delta}\left(\phi_{\delta}^{*} U_{n}, \chi_{\delta} v\right)\right| \leq \delta^{N-1} \sum_{n=N-3}^{N} \sum_{j=N+1-n}^{4}\left|\left\langle\phi_{\delta}^{*}\left(\nu^{j-2} A_{j} U_{n}\right), \frac{\chi_{\delta} v}{r^{2}}\right\rangle_{\Omega}\right| .
$$

To obtain a suitable estimate we only need to show that, for any $j=1 \ldots 4$ and any $n=0 \ldots N$ there exists a constant $C_{n, j}>0$ independent of $\delta$ such that $\forall v \in \mathrm{H}^{1}(\Omega), \forall \delta \in(0,1)$ we have

$$
\left|\left\langle\phi_{\delta}^{*}\left(\nu^{j-2} A_{j} U_{n}\right), \frac{\chi_{\delta} v}{r^{2}}\right\rangle_{\Omega}\right| \leq C_{n, j} \delta^{-1}\|v\|_{\mathrm{H}^{1}(\Omega)} .
$$

We show how to derive such a bound for $j=1$ (and so $n=N$ ). The case $j=2,3$ and 4 can be treated in a similar way, so there are left to the reader. 
We start by writing the decomposition of $U_{n}$ in the form "polynomial + evanescent": according to Proposition 5.2, there exist two functions $p_{ \pm}(\nu, \tau)$ that admit polynomial dependency with respect to $\nu$ with coefficients that depend on $\tau$ and belong to $C_{\#}^{\infty}\left(\mathbb{S}^{1}\right)$, and there exists a function $\mathfrak{U} \in C^{\infty}\left(\mathbb{S}^{1}, \mathbb{V}_{-}^{1}(B)\right)$ such that

$$
U_{n}(\nu, s, \tau)=P(\nu, \tau)+\mathfrak{U}(\nu, s, \tau) \quad \text { with } \quad P(\nu, \tau)=\chi(\nu) p_{+}(\nu, \tau)+\chi(-\nu) p_{-}(\nu, \tau) .
$$

In this decomposition $\chi: \mathbb{R} \rightarrow[0,1]$ is a $C^{\infty}(\mathbb{R})$ function such that $\chi=\chi(\nu), \chi(\nu)=1$ for $\nu>2 \pi$ and $\chi(\nu)=0$ for $\nu<\pi$. Plugging this decomposition into the term that we need to bound from above, we get

$$
\left\langle\phi_{\delta}^{*}\left(\nu^{-1} A_{1} U_{n}\right), \frac{\chi_{\delta} v}{r^{2}}\right\rangle_{\Omega}=\left\langle\phi_{\delta}^{*}\left(\nu^{-1} A_{1} P\right), \frac{\chi_{\delta} v}{r^{2}}\right\rangle_{\Omega}+\left\langle\phi_{\delta}^{*}\left(\nu^{-1} A_{1} \mathfrak{U}\right), \frac{\chi_{\delta} v}{r^{2}}\right\rangle_{\Omega} .
$$

Also, we will denote by $C_{\delta}$ the support of the truncation function $\chi_{\delta}$ :

$$
C_{\delta}:=\operatorname{supp}\left(\chi_{\delta}\right)=\left\{\mathbf{x} \in \mathbb{R}^{2}, r_{*}-2 \delta^{1-1 / N}<r<r_{*}+2 \delta^{1-1 / N}\right\} .
$$

Using the small size of $C_{\delta}$ we can prove the two following estimates (see for instance lemma 3.10 of [32]) : there exist two positive constants $C_{1}$ and $C_{2}$ such that

$$
\|u\|_{L^{1}\left(C_{\delta}\right)} \leq C_{1} \delta^{(N-1) / 2 N}\|u\|_{L^{2}\left(C_{\eta}\right)}, \quad\|u\|_{L^{2}\left(C_{\delta}\right)} \leq C_{2} \delta^{(N-1) / 2 N}\|u\|_{H^{1}(\Omega)} .
$$

Estimate of the first term We will successively derive an upper bound for each of the terms in the right hand side of $(88)$. We focus first on the term containing $P$. Observe that $\left(\nu^{-1} A_{1} P\right)(\nu, \tau)$ is a smooth function, both with respect to $\nu$ and $\tau$. Looking at the precise statement of Proposition 5.2 we see that the degree of $p_{ \pm}(\nu, \tau)$, as a polynomial in $\nu$, does not exceed $N$. In addition $P$ admits a smooth dependency with respect to $\tau$. As a consequence, we see that there exists a constant $C>0$ independent of $\delta$ such that

$$
\left|\left(\nu^{-1} A_{1} \cdot P\right)(\nu, \tau)\right| \leq C\left(1+|\nu|^{N-1}\right) \quad \forall \nu \in \mathbb{R}, \quad \forall \tau \in[0,2 \pi] .
$$

To establish the previous estimate we have used the fact that $A_{1}=\mathscr{A}_{1}$ when $|\nu|>\pi$ and the homogeneity of $\mathscr{A}_{1}$. Now observe that $\left|r-r_{*}\right| / \delta \leq 2 \frac{\eta(\delta)}{\delta} \leq 2 \delta^{-\frac{1}{N}}$ over $\operatorname{supp}\left(\chi_{\delta} v\right)$ according to the definition of $\chi_{\delta}$ given by (67). Since the change of variables induced by $\phi_{\delta}^{*}$ enforces $\nu=\left(r-r_{*}\right) / \delta$ and $\theta=\tau$, there exist constants $C_{1}, C_{2}>0$ independent of $\delta$ such that $\forall v \in \mathrm{H}^{1}(\Omega)$ and $\forall \delta \in(0,1)$ we have

$$
\left|\left\langle\phi_{\delta}^{*}\left(\nu^{-1} A_{1} P\right), \frac{\chi_{\delta} v}{r^{2}}\right\rangle_{\Omega}\right| \leq C_{1}\left(1+\delta^{-\frac{N-1}{N}}\right)\|v\|_{\mathrm{L}^{1}\left(C_{\delta}\right)} \leq C_{2}\|v\|_{\mathrm{H}^{1}(\Omega)} .
$$

Estimate of the second term Now we focus on the second term in $(88)$ that contains $\phi_{\delta}^{*}\left(\nu^{-1} A_{1} \mathfrak{U}\right)$. Let us rewrite this function in a different form. Using the definition of $A_{1}$ given by (25) and the relations (83), we have

$$
\begin{aligned}
\phi_{\delta}^{*}\left(\nu^{-1} A_{1} \mathfrak{U}\right)= & 2 r \partial_{r}\left[\phi_{\delta}^{*}\left(\epsilon^{-1} \partial_{\nu} \mathfrak{U}\right)\right]+\partial_{\theta}\left[\phi_{\delta}^{*}\left(\epsilon^{-1} \partial_{\tau} \mathfrak{U}\right)\right] \\
& +\phi_{\delta}^{*}\left[\epsilon^{-1} \partial_{\nu} \mathfrak{U}+\epsilon^{-1} \partial_{s} \partial_{\tau} \mathfrak{U}-\frac{\delta}{r_{*}} \epsilon^{-1} \partial_{\tau}^{2} \mathfrak{U}\right] .
\end{aligned}
$$

Since $\mathfrak{U} \in C_{\#}^{\infty}\left(\mathbb{S}^{1}, \mathbb{V}_{-}^{1}(B)\right)$ we have $g_{1}:=\epsilon^{-1} \partial_{\nu} \mathfrak{U} \in C_{\#}^{\infty}\left(\mathbb{S}^{1}, \mathbb{V}_{-}^{0}(B)\right)$ and $g_{2}:=\epsilon^{-1} \partial_{\tau} \mathfrak{U} \in C_{\#}^{\infty}\left(\mathbb{S}^{1}, \mathbb{V}_{-}^{0}(B)\right)$ on the one hand, and $g_{3}:=\epsilon^{-1} \partial_{\nu} \mathfrak{U}+\epsilon^{-1} \partial_{s} \partial_{\tau} \mathfrak{U}, g_{4}:=-\delta r_{*}^{-1} \epsilon^{-1} \partial_{\tau}^{2} \mathfrak{U} \in C^{\infty}\left(\mathbb{S}^{1}, \mathbb{V}_{-}^{0}(B)\right)$ on the other hand. Applying the definition of the weak derivative, the Cauchy-Schwarz inequality and the estimate [89], we obtain the existence of constants $C_{1}, C_{2}>0$ independent of $\delta$ such that

$$
\begin{aligned}
\left|\left\langle\phi_{\delta}^{*}\left(\nu^{-1} A_{1} \mathfrak{U}\right), \frac{\chi_{\delta} v}{r^{2}}\right\rangle_{\Omega}\right| & \leq C_{1}\left\|\frac{\chi_{\delta} v}{r^{2}}\right\|_{\mathrm{H}^{1}\left(C_{\delta}\right)} \sum_{i=1}^{4}\left\|\phi_{\delta}^{*} g_{i}\right\|_{\mathrm{L}^{2}\left(C_{\delta}\right)}, \\
& \leq C_{2} \delta^{-(N+1) / 2 N}\|v\|_{\mathrm{H}^{1}(\Omega)} \sum_{i=1}^{4}\left\|\phi_{\delta}^{*} g_{i}\right\|_{\mathrm{L}^{2}\left(C_{\delta}\right) .}
\end{aligned}
$$


In order to obtain a proper error estimate, there only remain to bound from above each $\left\|\phi_{\delta}^{*} g_{i}\right\|_{\mathrm{L}^{2}\left(C_{\delta}\right)}$. To do so, we use Estimate (68). Since $C_{\delta}$ has the same form as the set $K_{a}$ of this inequality with $a=2 \delta^{N-1 / N}$, this yields the existence of a constant $C>0$ independent of $\delta$ such that

$$
\left\|\phi_{\delta}^{*} g_{i}\right\|_{\mathrm{L}^{2}\left(C_{\delta}\right)}^{2} \leq C \sqrt{\delta}\left\|g_{i}\right\|_{\mathrm{L}^{2}\left(\mathbb{S}^{1}, \mathbb{V}_{-}^{0}(B)\right)}^{2} \quad \forall \delta \in(0,1), \quad i=1, \ldots, 4
$$

Now it suffices to plug (92) into (91). This provides a bound for $\left\langle\phi_{\delta}^{*}\left(\nu^{-1} A_{1} \mathfrak{U}\right), \frac{\chi_{\delta} v}{r^{2}}\right\rangle_{\Omega}$, which finally leads to the following estimate that we wanted to establish: there exists $C>0$ independent of $\delta$ such that

$$
\left|\left\langle\phi_{\delta}^{*}\left(\nu^{-1} A_{1} U_{n}\right), \frac{\chi_{\delta} v}{r^{2}}\right\rangle_{\Omega}\right| \leq C_{n, j} \delta^{-1 / 2 N}\|v\|_{\mathrm{H}^{1}(\Omega)} \quad \forall v \in \mathrm{H}^{1}(\Omega), \quad \forall \delta \in(0,1) .
$$

Summary Recall that $\left|\left\langle\phi_{\delta}^{*}\left(\nu^{j-2} A_{j} U_{n}\right), \chi_{\delta} v\right\rangle_{\Omega}\right|$ can be estimated using the same approach as the one we have just presented. As a consequence, coming back to Equation (87), this proves the following proposition .

Proposition 10.6 (Estimate for the near field error).

For a given $N \in \mathbb{N}$, let $\left(u_{0}, U_{0}\right), \ldots,\left(u_{N}, U_{N}\right)$ be the unique solutions to Problems (57)-(58)-(59). Let $\chi_{\delta}(\mathbf{x})=\chi\left(\left(r-r_{*}\right) / \eta(\delta)\right)$ where $\chi$ and $\eta(\delta)$ are defined in (67). Then there exists a constant $C, \delta_{0}>0$ independent of $\delta$ (depending on $N$ though) such that

$$
\left|\sum_{n=0}^{N} \delta^{n} a_{\delta}\left(\phi_{\delta}^{*} U_{n}, \chi_{\delta} v\right)\right| \leq C\|v\|_{\mathrm{H}^{1}(\Omega)} \delta^{N-2} \quad \forall v \in \mathrm{H}^{1}(\Omega), \quad \forall \delta \in\left(0, \delta_{0}\right) .
$$

\subsection{Conclusion of the error analysis}

Now we have at hand all the ingredients required for proving a global error estimate. Indeed this error estimate can be obtained by means of Proposition 10.3, as well as Equation (74) where we take into account Proposition 10.4 and 10.6 . This proves Theorem 10.1

\section{Conclusion}

Our study comes to an end. To conclude, let us remind the main results we obtained. First of all we described a complete asymptotic expansion of the solution of a scattering problem by a thin periodic layer. The approach is based on the well-known method of matched asymptotic expansion. We pay particular attention to write the matching conditions in an optimal way and to avoid the double recursion issue (that naturally appears in the transmission problems) by introducing new fields called Dirichlet and Neumann fields. These two main ingredients as well as a detailed analysis of the near field periodic problems allows us to prove quite easily the existence and uniqueness of the terms of the expansion. Besides, we also derived explicit formulas for the far and near field terms which can be very useful to construct approximate models up to any order. Finally, we proved the convergence of our expansion to the exact one with an optimal convergence rate. It would now be interesting to extend the previous approach to the more general case of a smooth thin and periodic interface. Whereas the calculations are more involved in this configuration, it seems that the mains issues of the analysis remain the same. Another interesting extension would be to generalize the method to the 3D Maxwell case, the analysis becoming much more complex due to the classical Maxwell Equations compactness issues. 


\section{Appendix}

\section{Lemma 11.1.}

Let $\psi_{\mathrm{R}}(\nu)=\psi(\nu / \mathrm{R})$ where $\psi$ is a $C^{\infty}$ function such that $\psi(\nu, s)=\psi(-\nu, s)=\psi(\nu)$, and $\psi(\nu)=0$ for $|\nu|>2 \pi$, and $\psi(\nu)=1$ for $|\nu|<\pi$. We have

$$
\int_{B} \nabla \mathfrak{U} \cdot \nabla \psi_{\mathrm{R}} d \nu d s=O\left(e^{-\pi \mathrm{R} / 2}\right), \quad \forall \mathfrak{U} \in \mathbb{V}_{-}^{1}(B) .
$$

Proof. Observe that $\left\|\nabla \psi_{\mathrm{R}}\right\|_{\mathbb{V}_{+}^{0}}=O\left(e^{-\pi \mathrm{R} / 2}\right)$ for $\mathrm{R} \rightarrow+\infty$. As a consequence, by Cauchy-Schwarz inequality, there exists $C>0$ depending only on $\psi$ such that $\int_{B} \epsilon^{-1} \nabla \mathfrak{U} \cdot \nabla \psi_{\mathrm{R}} d \nu d s \leqslant C\|\mathfrak{U}\|_{\mathbb{V}_{-}^{1}} e^{-\pi \mathrm{R} / 2}$ $\forall \mathfrak{U} \in \mathbb{V}_{-}^{1}(B)$.

\section{Proposition 4.7}

Assume that $U \in \mathbb{V}_{+}^{1}(B)$ satisfies for $-\nabla \cdot\left(\epsilon^{-1} \nabla U\right)=g$ in $\mathbb{V}_{+}^{1}(B)^{\prime}$ for some $g \in \mathbb{V}_{+}^{1}(B)^{\prime}$. Then we have

$$
\left[\ell_{\mathrm{N}}(U)\right]=-\frac{\epsilon_{\infty}}{2 \pi}\langle g, 1\rangle_{+} \quad \text { and } \quad\left[\ell_{\mathrm{D}}(U)\right]=\left\langle\ell_{\mathrm{N}}(U)\right\rangle \cdot\left[\ell_{\mathrm{D}}(\mathcal{N})\right]-\frac{\epsilon_{\infty}}{2 \pi}\langle g, \mathcal{N}\rangle_{+} .
$$

Proof. Take a cut-off function $\psi(\nu, s)=\psi(-\nu, s)=\psi(\nu)$, and $\psi(\nu)=0$ for $|\nu|>2 \pi$, and $\psi(\nu)=1$ for $|\nu|<\pi$. Define $\psi_{\alpha}(\nu)=\psi(\nu / \alpha)$. The dominated convergence theorem shows that $\psi_{\alpha}$ converges toward 1 in $\mathbb{V}_{+}^{1}(B)$. As a consequence we have in particular $\lim _{\alpha \rightarrow \infty}<g, \psi_{\alpha}>_{+}=<g, 1>_{+}$. Define $B_{\alpha}^{ \pm}=\{(\nu, s)|\pi \alpha< \pm \nu<2 \pi \alpha| s \mid,<\pi\}$. We have

$$
\int_{B} \epsilon^{-1} \nabla U \cdot \nabla \psi_{\alpha} d \nu d s=\epsilon_{\infty}^{-1}\left[\int_{B_{\alpha}^{+}} \nabla U \cdot \nabla \psi_{\alpha} d \nu d s+\int_{B_{\alpha}^{-}} \nabla U \cdot \nabla \psi_{\alpha} d \nu d s\right] .
$$

Since $-\nabla \cdot\left(\epsilon^{-1} \nabla U\right)=g$ in $\mathbb{V}_{+}^{1}(B)^{\prime}$, according to Proposition 4.3 there exists $\mathfrak{U} \in \mathbb{V}_{-}^{1}(B)$ such that $U(\nu, s)=\ell_{\mathrm{D}}^{+}(U)+\ell_{\mathrm{N}}^{+}(U) \nu+\mathfrak{U}(\nu, s)$ for $\nu>2 \pi$. Plugging this decomposition into the first integral in the right hand side of (93), and using Lemma 11.1 yields

$$
\int_{B_{\alpha}^{+}} \nabla U \cdot \nabla \psi_{\alpha} d \nu d s=\ell_{\mathrm{N}}^{+}(U) 2 \pi \int_{\pi \alpha}^{2 \pi \alpha} \partial_{\nu} \psi_{\alpha} d \nu+O\left(e^{-\alpha / 2}\right) \underset{\alpha \rightarrow+\infty}{\longrightarrow}-2 \pi \ell_{\mathrm{N}}^{+}(U) .
$$

Using similar arguments, we also prove that $\lim _{\alpha \rightarrow \infty} \int_{B_{\alpha}^{-}} \nabla U \cdot \nabla \psi_{\alpha} d \nu d s=2 \pi \ell_{\mathrm{N}}^{-}(U)$. Gathering these two asymptotic results proves the first identity.

In the same manner we can prove the second identity. First, by the dominated convergence theorem it is easily seen that $\lim _{\alpha \rightarrow+\infty}\left\langle g, \psi_{\alpha} \mathcal{N}\right\rangle_{+}=\langle g, \mathcal{N}\rangle_{+}$. But, since $\int_{B} \nabla\left(\psi_{\alpha} U\right) \cdot \nabla \mathcal{N} d \nu d s=0$,

$$
\left\langle g, \psi_{\alpha} \mathcal{N}\right\rangle_{+}=\int_{B} \nabla U \cdot \nabla\left(\psi_{\alpha} \mathcal{N}\right) d \nu d s=\int_{B} \mathcal{N} \nabla U \cdot \nabla \psi_{\alpha}-\mathcal{U} \nabla \mathcal{N} \cdot \nabla \psi_{\alpha} d \nu d s
$$

Moreover, according to Proposition 4.3 we can see that

$$
\lim _{\alpha \rightarrow+\infty} \int_{B} \mathcal{N} \nabla U \cdot \nabla \psi_{\alpha}-\mathcal{U} \nabla \mathcal{N} \cdot \nabla \psi_{\alpha} d \nu d s=2 \pi \epsilon_{\infty}^{-1}\left(\ell_{\mathrm{N}}^{+}(U) \ell_{\mathrm{D}}^{+}(\mathcal{N})-\ell_{\mathrm{N}}^{-}(U) \ell_{\mathrm{D}}^{-}(\mathcal{N})-\left[\ell_{\mathrm{D}}(U)\right]\right) .
$$

Reminding that $\left\langle\ell_{\mathrm{D}}(\mathcal{N})\right\rangle=0$, we obtain the desired formula:

$$
\langle g, \mathcal{N}\rangle_{+}=2 \pi \epsilon_{\infty}^{-1}\left(-\left[\ell_{\mathrm{D}}(U)\right]+\left[\ell_{\mathrm{N}}(U)\right]\left\langle\ell_{\mathrm{D}}(\mathcal{N})\right\rangle\right) .
$$




\section{Lemma 11.2.}

The operator $T_{\#}$ defined by $\left(T_{\#} \varphi\right)(\nu, s)=\sum_{k \in \mathbb{Z}} \varphi(\nu, s+2 k \pi)$ maps $\mathscr{D}\left(\mathbb{R}^{2}\right)$ in $\mathscr{D} \#(B)$ and is onto.

Proof. We have to prove that for any $\varphi \in \mathscr{D} \#(B)$ there exists $v \in \mathscr{D}\left(\mathbb{R}^{2}\right)$ such that $T_{\#} v=\varphi$. Assume first that $\varphi \in \mathscr{D}((0,2 \pi) \times \mathbb{R})$, so that $\varphi(\nu, s)=0$ if $0 \leq s<\varepsilon$ or $2 \pi-\varepsilon<s \leq 2 \pi$ for some $\varepsilon>0$. We extend $\varphi$ by 0 outside $(0,2 \pi) \times \mathbb{R}$, so that $\varphi \in \mathscr{D}\left(\mathbb{R}^{2}\right)$. Denote $\left(\tau_{\alpha} \varphi\right)(\nu, s)=\varphi(\nu, \alpha+s)$. We have $\operatorname{supp}\left(\tau_{2 k \pi} \varphi\right) \cap \operatorname{supp}(\varphi)=\emptyset$ except if $k \neq 0$. As a consequence, defining $v(\nu, s)$ by $v(\nu, s)=\varphi(\nu, s)$ if $0 \leq s \leq 2 \pi$, and $v(\nu, s)=0$ if $s<0$ or $s>2 \pi$, we have $v \in \mathscr{D}\left(\mathbb{R}^{2}\right)$ and $T_{\#} v=\varphi$.

Now take any $\varphi \in \mathscr{D}_{\#}(B)$. Using a cut-off function and the special case that we have just examined, we may consider in addition that $\varphi(\nu, s)=0$ if $\pi / 3<s<5 \pi / 3$. Introduce a $C^{\infty}$ cut-off function $\chi: \mathbb{R} \rightarrow \mathbb{R}_{+}$such that $\chi(s)=1$ if $0<s<\pi / 3$ and $\chi(s)=0$ if $2 \pi / 3<s$, and define $v(\nu, s)$ in the following manner: $v(\nu, s)=0$ if $s>2 \pi / 3$ or $s<-2 \pi / 3$, and $v(\nu, s)=\chi(s) \varphi(\nu, s)$ if $0 \leq s \leq 2 \pi / 3$, and $v(\nu, s)=\chi(-s) \varphi(\nu, 2 \pi+s)$ if $-2 \pi / 3 \leq s \leq 0$. With such a definition for $v$, we have $T_{\#} v=\varphi$ once again.

\section{Lemma 11.3.}

The space $\mathscr{D}_{\#}(B)$ is dense in $\mathbb{W}(B)$ for the norm \|\| $\mathbb{w}$, and it is dense in $\mathbb{V}_{+}^{1}(B)$ for the norm \|\|$_{\left(\mathbb{V}_{+}^{1}\right)}$.

Proof. Consider any $\varphi \in \mathbb{V}_{+}^{1}(B)$ chosen arbitrarily. Take a $C^{\infty}$ cut-off function $\chi: \mathbb{R} \rightarrow \mathbb{R}_{+}$such that $\chi(\nu)=1$ if $|\nu|<1$ and $\chi(\nu)=0$ if $|\nu|>2$. Set $\chi_{k}(\nu)=\chi(\nu / k)$. The dominated convergence theorem shows that $\lim _{k \rightarrow \infty}\left\|\varphi-\chi_{k} \varphi\right\|_{\mathbb{V}_{+}^{1}}=0$. On the other hand, if $\varphi \in \mathbb{W}(B)$, and if $\chi_{k}$ refers to the same cutoff function as above, dominated convergence theorem shows once again that $\lim _{k \rightarrow \infty}\left\|\varphi-\chi_{k} \varphi\right\|_{\mathbb{W}}=0$. Let

$$
\mathrm{H}_{\#, \mathrm{c}}^{1}(B)=\left\{\varphi \in \mathrm{H}_{\#}^{1}(B) \mid \exists \nu_{0}>0 \text { such that } \varphi(\nu)=0 \text { for }|\nu|>\nu_{0}\right\} .
$$

Observe that \|\|$_{\mathbb{V}_{+}^{1}},\|\|_{\mathbb{W}}$ and \|\|$_{\mathrm{H}^{1}(B)}$ all induce the same topology over $\mathrm{H}_{\#, \mathrm{c}}^{1}(B)$. As a consequence, to prove the lemma, it is sufficient to show that any element of $\mathrm{H}_{\#, \mathrm{c}}^{1}(B)$ can be approximated by functions chosen in $\mathscr{D}_{\#}(B)$. From now on, let us consider a function $\varphi \in \mathrm{H}_{\#, \mathrm{c}}^{1}(B)$. Since $\varphi$ has compact support, it suffices to prove that there exists a sequence $v_{n} \in \mathscr{D}_{\#}(B), n \in \mathbb{N}$ such that $\lim _{n \rightarrow \infty}\left\|\varphi-v_{n}\right\|_{\mathrm{H}^{1}(B)}=0$. Let us decompose $\varphi$ in a Fourier series with respect to the variable $s$.

$$
\varphi(\nu, s)=\sum_{k=-\infty}^{+\infty} \alpha_{k}(\nu) e^{i k s} \quad \text { with } \quad \alpha_{k}(\nu)=\frac{1}{2 \pi} \int_{0}^{2 \pi} \varphi(\nu, s) e^{-i k s} d s, k \in \mathbb{Z} .
$$

Note that each $\alpha_{k}(\nu)$ belongs to $\mathrm{H}^{1}(\mathbb{R})$ and satisfies $\alpha_{k}(\nu)=0$ whenever $|\nu|>\nu_{0}$ for any $k$. Besides the series above converges in $\mathrm{H}^{1}(B)$. Indeed set $\varphi_{N}(\nu, s)=\sum_{|k| \leq N} \alpha_{k}(\nu) e^{i k s}$. We have $\lim _{N \rightarrow \infty} \| \varphi-$ $\varphi_{N} \|_{\mathrm{H}^{1}(B)}=0$.

Finally take an arbitrary $\varepsilon>0$. There exists $N \geq 1$ such that $\left\|\varphi-\varphi_{N}\right\|_{\mathrm{H}^{1}(B)} \leq \varepsilon$. Besides, since $\mathscr{D}(\mathbb{R})=\left\{v \in C^{\infty}(\mathbb{R}) \mid \operatorname{supp}(v)\right.$ is compact $\}$ is dense in $\mathrm{H}^{1}(\mathbb{R})$, there exists $\beta_{k} \in \mathscr{D}(\mathbb{R})$ such that $\left\|\alpha_{k}-\beta_{k}\right\|_{\mathrm{H}^{1}(\mathbb{R})}^{2}<\varepsilon^{2} /\left(4 N^{3}\right)$. Clearly $\beta_{k}(\nu) e^{i k s} \in \mathscr{D}_{\#}(B)$ for each $k=-N, \ldots,+N$. Besides, an easy calculus yields $\left\|\left(\alpha_{k}(\nu)-\beta_{k}(\nu)\right) e^{i k s}\right\|_{\mathrm{H}^{1}(B)}^{2} \leq \varepsilon^{2} / N$ for any $k=-N, \ldots,+N$. Setting $v=$ $\sum_{|k| \leq N} \beta_{k}(\nu) e^{i k s}$, we obtain

$$
\left\|\varphi_{N}-v\right\|_{\mathrm{H}^{1}(B)}^{2}=\sum_{k=-N}^{N}\left\|\left(\alpha_{k}(\nu)-\beta_{k}(\nu)\right) e^{i k s}\right\|_{\mathrm{H}^{1}(B)}^{2} \leq \frac{2 N+1}{N} \varepsilon^{2} \leq 4 \varepsilon^{2} .
$$

Clearly $v \in \mathscr{D}_{\#}(B)$. As a consequence, to sum up, for an arbitrary $\varepsilon>0$, we have constructed a function $v \in \mathscr{D}_{\#}(B)$ such that $\|\varphi-v\|_{\mathrm{H}^{1}(B)} \leq\left\|\varphi-\varphi_{N}\right\|_{\mathrm{H}^{1}(B)}+\left\|\varphi_{N}-v\right\|_{\mathrm{H}^{1}(B)} \leq 3 \varepsilon$. This concludes the proof of the density of $\mathscr{D}_{\#}(B)$ in $\mathbb{V}_{+}^{1}(B)$. 


\section{Proposition 11.4.}

For $g \in \mathbb{V}_{+}^{1}(B)^{\prime}$ and $\alpha, \beta \in \mathbb{C}$, let $U \in \mathbb{V}_{+}^{1}(B)$ be the unique solution to Problem (42). Let $\chi: \mathbb{R} \rightarrow[0,1]$ be a $C^{\infty}$ cut-off function that satisfies $\chi(\nu)=0$ for $\nu<\pi$ and $\chi(\nu)=1$ for $\nu>2 \pi$. Finally let $\mathfrak{U} \in \mathbb{V}_{-}^{1}(B)$ be defined by

$$
\begin{aligned}
& \mathfrak{U}(\nu, s)=U(\nu, s)-P(\nu) \\
& \text { where } P(\nu)=\chi(\nu)\left[\ell_{\mathrm{D}}^{+}(U)+\ell_{\mathrm{N}}^{+}(U) \nu\right]+\chi(-\nu)\left[\ell_{\mathrm{D}}^{-}(U)+\ell_{\mathrm{N}}^{-}(U) \nu\right] .
\end{aligned}
$$

Then there is continuous dependency of $\mathfrak{U}$ with respect to $\alpha, \beta, g$ : there exists a constant $C>0$ independent of $\alpha, \beta, g$ such that

$$
\|\mathfrak{U}\|_{\mathbb{V}_{-}^{1}} \leq C\left(|\alpha|+|\beta|+\|g\|_{\left(\mathbb{V}_{+}^{1}\right)^{\prime}}\right) .
$$

Proof. Set $\chi(\nu)=\chi_{-}(\nu)+\chi_{+}(\nu)$ and $\psi(\nu)=1-\chi(\nu)$. Set $U_{ \pm}=\chi_{ \pm} U$ and $U_{0}=\psi U$. Let us set also $g_{ \pm}=-\nabla \cdot\left(\epsilon^{-1} \nabla U_{ \pm}\right)$and $g_{0}=-\nabla \cdot\left(\epsilon^{-1} \nabla U_{0}\right)$. Then clearly $g_{ \pm}, g_{0} \in \mathbb{V}_{+}^{1}(B)^{\prime}$ and, using (46), it is easy to establish that there exists $C>0$ independent of $g$ such that

$$
\left\|g_{0}\right\|_{\left(\mathbb{V}_{+}^{1}\right)^{\prime}}+\left\|g_{+}\right\|_{\left(\mathbb{V}_{+}^{1}\right)^{\prime}}+\left\|g_{-}\right\|_{\left(\mathbb{V}_{+}^{1}\right)^{\prime}} \leq C\left(|\alpha|+|\beta|+\|g\|_{\left(\mathbb{V}_{+}^{1}\right)^{\prime}}\right) .
$$

Besides, by linearity, we have $g=g_{0}+g_{+}+g_{-}$. Denote by $\mathfrak{U}_{ \pm}, \mathfrak{U}_{0} \in \mathbb{V}_{-}^{1}(B)$ the remainder terms associated respectively with $U_{+}, U_{-}$and $U_{0}$ following the statement of the proposition we want to establish. Clearly we have $\mathfrak{U}=\mathfrak{U}_{+}+\mathfrak{U}_{-}+\mathfrak{U}_{0}$. All these preliminary remarks show that, to prove the proposition, it suffices to prove that there exists $C>0$ independent of $\alpha, \beta, g$ such that

$$
\begin{aligned}
& \text { i) }\left\|\mathfrak{U}_{0}\right\|_{\mathbb{V}_{-}^{1}} \leq C\left(|\alpha|+|\beta|+\|g\|_{\left(\mathbb{V}_{+}^{1}\right)^{\prime}}\right) \text {, } \\
& \text { ii) }\left\|\mathfrak{U}_{+}\right\|_{\mathbb{V}_{-}^{1}} \leq C\left(\left|\ell_{\mathrm{D}}^{+}\left(U_{+}\right)\right|+\left|\ell_{\mathrm{N}}^{+}\left(U_{+}\right)\right|+\left\|g_{+}\right\|_{\left(\mathbb{V}_{+}^{1}\right)^{\prime}}\right) \text {. }
\end{aligned}
$$

The derivation of an estimate for $\mathfrak{U}_{-}$would follow the same lines as the proof of ii) so we skip it. Note also that $\ell_{\mathrm{D}}^{+}\left(U_{+}\right)=\ell_{\mathrm{D}}^{+}(U)$ and $\ell_{\mathrm{N}}^{+}\left(U_{+}\right)=\ell_{\mathrm{N}}^{+}(U)$ so that according to Proposition 4.7 there exists $C>0$ independent of $\alpha, \beta, g$ such that $\left|\ell_{\mathrm{D}}^{+}\left(U_{+}\right)\right|+\left|\ell_{\mathrm{N}}^{+}\left(U_{+}\right)\right| \leq C\left(|\alpha|+|\beta|+\|g\|_{\left(\mathbb{V}_{+}^{1}\right)^{\prime}}\right)$.

Proof of i) Observe that $\mathfrak{U}_{0}=U_{0}$ since $\ell_{\mathrm{D}}^{ \pm}\left(U_{0}\right)=0$ and $\ell_{\mathrm{N}}^{ \pm}\left(U_{0}\right)=0$, since $U_{0}(\nu, s)=0$ for $|\nu|>2 \pi$. Besides there exists a constant $C>0$ such that $\|\psi U\|_{\mathbb{V}_{-}^{1}} \leq C\|U\|_{\mathbb{V}_{+}^{1}}$. Thus i) is actually a consequence of (46).

Proof of ii) Let $\phi(\nu)=\chi_{+}(\nu / 2)$, so that $\phi(\nu)=0$ for $\nu<2 \pi$ and $\phi(\nu)=1$ for $\nu>4 \pi$. Since $\mathfrak{U}_{+}(\nu, s)=0$ for $\nu \leq \pi$, according to (46) and (47), there exists a constant $C>0$ independent of $\ell_{\mathrm{D}}^{+}\left(U_{+}\right), \ell_{\mathrm{N}}^{+}\left(U_{+}\right), g_{+}$such that the following inequalities hold

$$
\left\|\mathfrak{U}_{+}\right\|_{\mathbb{V}_{-}^{1}}^{2} \leq e^{4 \pi}\left(\left\|\mathfrak{U}_{+}\right\|_{\mathbb{V}_{+}^{1}}^{2}+\left\|\phi \mathfrak{U}_{+}\right\|_{\mathbb{V}_{-}^{1}}^{2}\right) \leq C\left(\left|\ell_{\mathrm{D}}^{+}\left(U_{+}\right)\right|+\left|\ell_{\mathrm{N}}^{+}\left(U_{+}\right)\right|+\left\|g_{+}\right\|_{\left(\mathbb{V}_{+}^{1}\right)^{\prime}}+\left\|\phi \mathfrak{U}_{+}\right\|_{\mathbb{V}_{-}^{1}}^{2}\right) .
$$

As a consequence, it is sufficient to provide an upper bound for $\left\|\phi \mathfrak{U}_{+}\right\|_{\mathbb{V}^{1}}$ in order to prove ii). Now let us come back to the proof of Proposition 4.3. Denote $\hat{g}_{+, k}(\lambda)=\left\langle g_{+}, \exp (-\lambda \nu-i k s)\right\rangle_{+}$. According to Equation [39), we have $\phi(\nu) \mathfrak{U}_{+}(\nu, s)=\phi(\nu) \mathfrak{V}_{+}(\nu, s)$ for $\nu \geq 2 \pi$ where

$$
\mathfrak{V}_{+}(\nu, s)=\sum_{k \in \mathbb{Z}} \frac{1}{2 i \pi} \int_{\Re e\{\lambda\}=-\frac{1}{2}} \frac{\hat{g}_{+, k}(\lambda)}{k^{2}-\lambda^{2}} e^{\lambda \nu+i k s} d \lambda .
$$

However $\mathfrak{U}_{+}(\nu, s) \neq \mathfrak{V}_{+}(\nu, s)$ a priori for $\nu<2 \pi$. Elementary estimates then show that there exist constants $C_{1}, C_{2}>0$ independent of $\ell_{\mathrm{D}}^{+}\left(U_{+}\right), \ell_{\mathrm{N}}^{+}\left(U_{+}\right), g_{+}$such that

$$
\left\|\phi \mathfrak{U}_{+}\right\|_{\mathbb{V}^{1}-}^{2} \leq C_{1}\|\phi \mathfrak{V}\|_{\mathbb{V}_{-}^{1}}^{2} \leq C_{2}\left\|e^{\frac{\nu}{2}} \mathfrak{V}\right\|_{\mathrm{H}^{1}(B)}^{2} .
$$


We conclude the proof by using estimates provided by (38) and (40): there exist constants $C_{3}, C_{4}>0$ independent of $\ell_{\mathrm{D}}^{+}\left(U_{+}\right), \ell_{\mathrm{N}}^{+}\left(U_{+}\right), g_{+}$such that

$$
\left\|e^{\frac{\nu}{2}} \mathfrak{V}\right\|_{\mathrm{H}^{1}(B)}^{2} \leq C_{3} \sum_{k=-\infty}^{+\infty} \int_{-\infty}^{+\infty} \frac{\left|\hat{g}_{+, k}(-1 / 2+i \xi)\right|^{2}}{1+k^{2}+\xi^{2}} d \xi \leq C_{4}\left\|g_{+}\right\|_{\left(\mathbb{V}_{+}^{1}\right)^{\prime}}^{2} .
$$

\section{References}

[1] T. Abboud and H. Ammari. Diffraction at a curved grating: TM and TE cases, homogenization. $J$. Math. Anal. Appl., 202(3):995-1026, 1996.

[2] Y. Achdou. Etude de la réflexion d'une onde électromagnétique par un métal recouvert d'un revêtement métallisé. Technical report, INRIA, 1989.

[3] G. Allaire. Shape optimization by the homogenization method, volume 146 of Applied Mathematical Sciences. Springer-Verlag, New York, 2002.

[4] H. Ammari and C. Latiri-Grouz. Conditions aux limites approchées pour les couches minces périodiques. M2AN Math. Model. Numer. Anal., 33(4):673-693, 1999.

[5] M. Artola and M. Cessenat. Diffraction d'une onde électromagnétique par une couche composite mince accolée à un corps conducteur épais. I. Cas des inclusions fortement conductrices. C. R. Acad. Sci. Paris Sér. I Math., 313(5):231-236, 1991.

[6] A. Bensoussan, J.L. Lions, and G. Papanicolaou. Asymptotic analysis for periodic structures, volume 5 of Studies in Mathematics and its Applications. North-Holland Publishing Co., Amsterdam, 1978.

[7] E. Bonnetier, D. Bresch, and V. Milišić. A priori convergence estimates for a rough Poisson-Dirichlet problem with natural vertical boundary conditions. Advances in Mathematical Fluid Mechanics, pages $105-134,2010$

[8] D. Bresch and V. Milisic. High order multi-scale wall-laws, part I: The periodic case. Arxiv preprint math/0611083, 2006.

[9] I. S. Ciuperca, M. Jai, and C. Poignard. Approximate transmission conditions through a rough thin layer: the case of periodic roughness. European J. Appl. Math., 21(1):51-75, 2010.

[10] X. Claeys. Analyse asymptotique et numérique de la diffraction d'ondes par des fils minces. $\mathrm{PhD}$ thesis, Université Versailles St Quentin, 2008.

[11] D. Colton and R. Kress. Inverse acoustic and electromagnetic scattering theory, volume 93 of Applied Mathematical Sciences. Springer-Verlag, Berlin, second edition, 1998.

[12] Monique Dauge, Erwan Faou, and Victor Péron. Comportement asymptotique à haute conductivité de l'épaisseur de peau en électromagnétisme. C. R. Math. Acad. Sci. Paris, 348(7-8):385-390, 2010.

[13] B. Delourme, H. Haddar, and P. Joly. Approximate models for wave propagation across thin periodic interfaces. Journal de mathématiques pures et appliquées, 2012.

[14] D. Drissi. Simulation des silencieux d'échappement par une méthode d'éléments finis homogénéisés. PhD thesis, Université de Tunis, 2003.

[15] R. R. Gadyl'shin. The method of matching asymptotic expansions in a singularly perturbed boundary value problem for the Laplace operator. Sovrem. Mat. Prilozh., (5, Asimptot. Metody Funkts. Anal.):3-32, 2003. 
[16] H. Haddar, P. Joly, and H.M. Nguyen. Generalized impedance boundary conditions for scattering by strongly absorbing obstacles: the scalar case. Math. Models Methods Appl. Sci., 15(8):1273-1300, 2005 .

[17] A. M. Il'in. Matching of asymptotic expansions of solutions of boundary value problems, volume 102 of Translations of Mathematical Monographs. American Mathematical Society, Providence, RI, 1992. Translated from the Russian by V. Minachin [V. V. Minakhin].

[18] A. M. Il'in, A. R. Danilin, and S. V. Zakharov. Application of the method of matching asymptotic expansions to the solution of boundary value problems. Sovrem. Mat. Prilozh., (5, Asimptot. Metody Funkts. Anal.):33-78, 2003.

[19] P. Joly and S. Tordeux. Matching of asymptotic expansions for wave propagation in media with thin slots. I. The asymptotic expansion. Multiscale Model. Simul., 5(1):304-336 (electronic), 2006.

[20] P. Joly and S. Tordeux. Matching of asymptotic expansions for waves propagation in media with thin slots. II. The error estimates. M2AN Math. Model. Numer. Anal., 42(2):193-221, 2008.

[21] V. A. Kozlov, V. G. Mazya, and J. Rossmann. Elliptic boundary value problems in domains with point singularities, volume 52 of Mathematical Surveys and Monographs. American Mathematical Society, Providence, RI, 1997.

[22] A.L. Madureira and F. Valentin. Asymptotics of the Poisson problem in domains with curved rough boundaries. SIAM J. Math. Anal., 38(5):1450-1473 (electronic), 2006/07.

[23] V. Maz'ya, S. Nazarov, and B. Plamenevskij. Asymptotic theory of elliptic boundary value problems in singularly perturbed domains. Vol. I, volume 111 of Operator Theory: Advances and Applications. Birkhäuser Verlag, Basel, 2000. Translated from the German by Georg Heinig and Christian Posthoff.

[24] W. McLean. Strongly elliptic systems and boundary integral equations. Cambridge University Press, Cambridge, 2000.

[25] J.C. Nédélec. Acoustic and electromagnetic equations, volume 144 of Applied Mathematical Sciences. Springer-Verlag, New York, 2001. Integral representations for harmonic problems.

[26] J.-R. Poirier, A. Bendali, P. Borderies, and S. Tournier. High order asymptotic expansion for the scattering of fast oscillating periodic surfaces. In proceedings of waves 2009, 2009.

[27] W. Rudin. Functional analysis. International Series in Pure and Applied Mathematics. McGraw-Hill Inc., New York, second edition, 1991.

[28] E. Sánchez-Palencia. Nonhomogeneous media and vibration theory, volume 127 of Lecture Notes in Physics. Springer-Verlag, Berlin, 1980.

[29] E. Sánchez-Palencia. Un problème d' écoulement lent d'une fluide visqueux incompressible au travers d'une paroi finement perforée. Technical report, Electricité de France, 1985.

[30] K. Schmidt and S. Tordeux. Asymptotic modelling of conductive thin sheets. Zeitschrift $f$ "ur Angewandte Mathematik und Physik (ZAMP), 61(4):603-626, 2010.

[31] M. Tlemcani. A Two-Scale Asymptotic Analysis of a Time-Harmonic Scattering Problem with a Multi Layered Thin Periodic Domain. Communications in Computational Physics, 6:758-776, 2009.

[32] S. Tordeux. Méthode asymptotiques pour la propagation des ondes dans des milieux comportant des fentes. PhD thesis, Université Versailles St Quentin, 2004.

[33] M. Van Dyke. Perturbation methods in fluid mechanics. Applied Mathematics and Mechanics, Vol. 8. Academic Press, New York, 1964.

[34] A. Zebic. Conditions de frontière équivalentes en électromagnétisme. PhD thesis, Université Paris 6, 1994. 


\section{List of Figures}

$1 \quad$ The thin and periodic ring . . . . . . . . . . . . . . . . . . . 41

2 Far and near fields domains . . . . . . . . . . . . . . . . . . . . 42

3 Localization of far field and near field expansions . . . . . . . . . . . . . . . . 43 


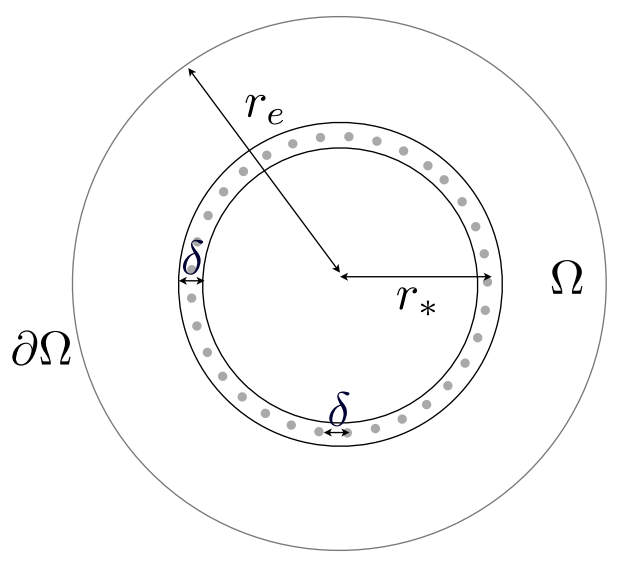

(a) Multi-scale geometry

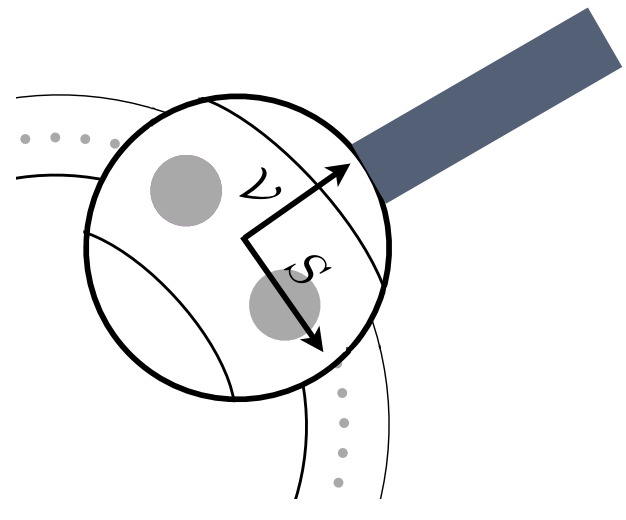

(b) Scaled coordinates

Figure 1: The thin and periodic ring 


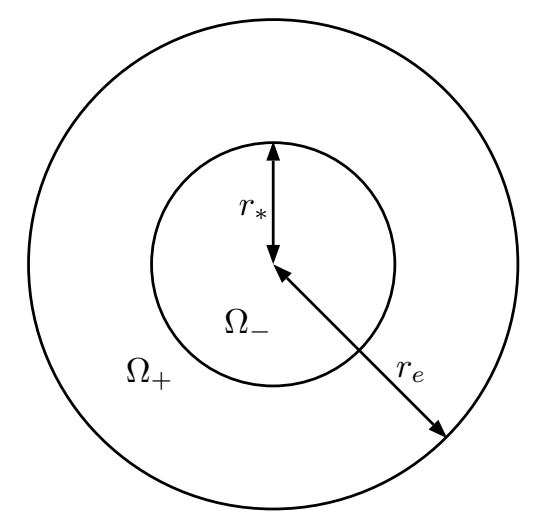

(a) Domain of the far field

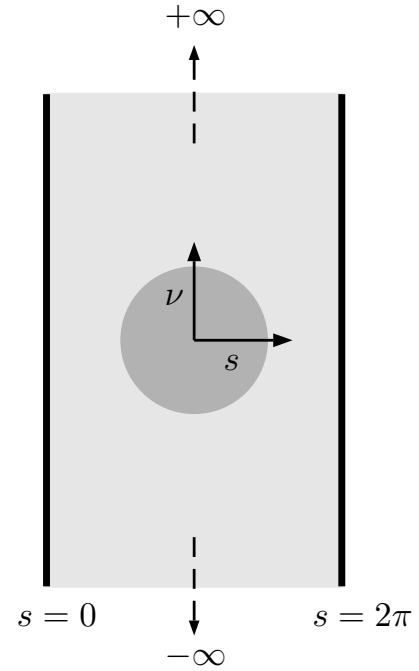

(b) Domain of the near field

Figure 2: Far and near fields domains 


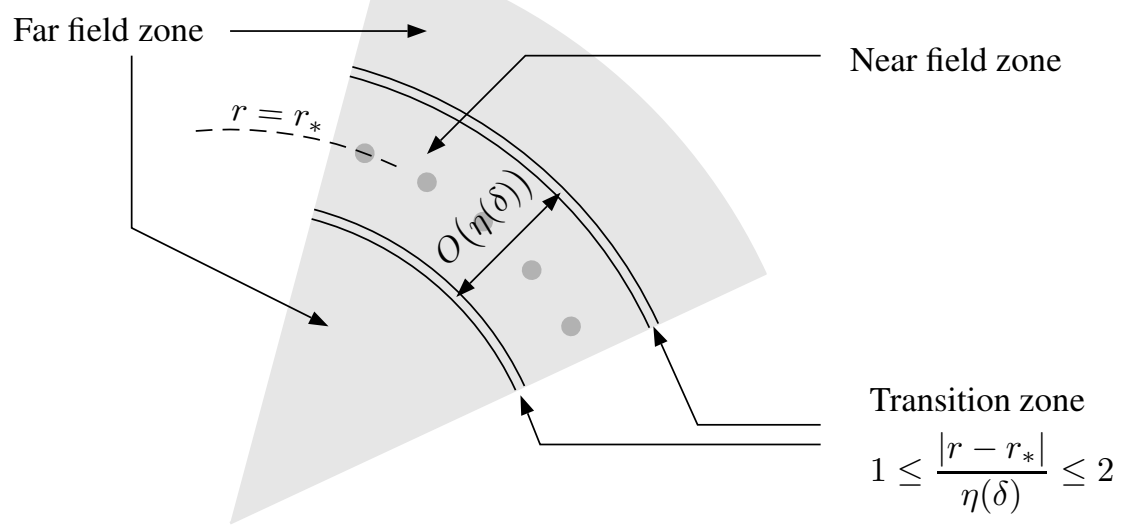

Figure 3: Localization of far field and near field expansions 